\title{
Book of Abstracts
}

\section{Microbiology Centennial Symposium 2017}

'Exploring Microbes for the Quality of Life'

\author{
Editors: \\ Willem M. de Vos \\ Hauke Smidt \\ John van der Oost \\ Fons Stams \\ Servé Kengen
}

18 - 20 October 2017

Wageningen

The Netherlands 
Microbiology Centennial Symposium 2017

'Exploring Microbes for the Quality of Life'

18 - 20 October 2017

Wageningen

The Netherlands

Editors: Willem M. de Vos, Hauke Smidt, John van der Oost, Fons Stams, Servé Kengen

Wageningen University \& Research, PO Box 8033, 6700 EH Wageningen, The Netherlands

Quotation from or reference to any part of this book should be made with full reference to the above data

Cover design: Wageningen University \& Research, Communication Services

ISBN: $\quad 978-94-6343-808-7$

DOI: $\quad$ https://doi.org/10.18174/424506

Design: Wageningen University \& Research, Communication Services, Wageningen, The Netherlands 
Welcome 


\section{Welcome}

\section{Exploring Microbes}

The research of the Laboratory of Microbiology of Wageningen University \& Research has been quite diverse. Since the early days there has been a focus on fundamental aspects together with a strong relation with societal issues. This has continued to this date and is reflected in the Microbiology Centennial Symposium title 'Exploring Microbes for the Quality of Life'.

For the last 25 years we have focused on molecular ecology, microbial physiology and bacterial genetics and notably their integration. These subject areas will form the core of the scientific program that is initiated by overviews of microbial interactions with the biotic and abiotic environment and is closed with an outlook on the future.

Microbial research overview

The keynote lectures include eminent scientists (see below) who have been interacting with the Laboratory of Microbiology throughout the years. The program also features the present research group leaders of the Laboratory of Microbiology as well as the present and past chair who all have contributed to the success of the last decades.

On behalf of the scientific organising committee, I welcome you all to participate in this exciting symposium that will provide you with a state of the art overview of many important areas in microbial research, varying from novel anaerobic conversions to intestinal microbiology and extremophiles research to genome editing.

Willem M. de Vos

Professor and Chair

Microbiology Centennial Symposium 2017 


\section{Contents}

Welcome....................................................................................................... 3

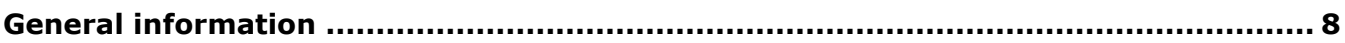

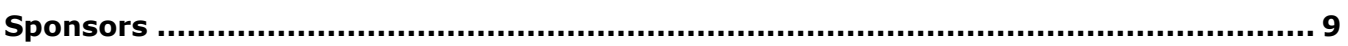

Organising Committee ........................................................................................... 10

Conference Secretariat ............................................................................... 10

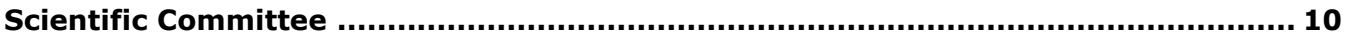

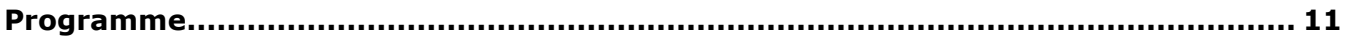

Brief introduction - Keynote speakers............................................................... 17

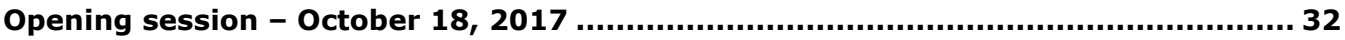

Jan Roelof van der Meer - What can we learn from microbes in the environment ................................... 33

Mark van Loosdrecht - Microbiology at the interphase ................................................................ 34

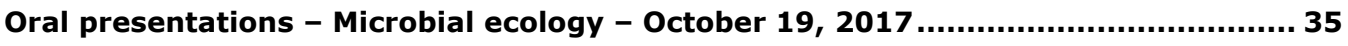

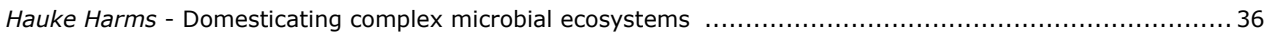

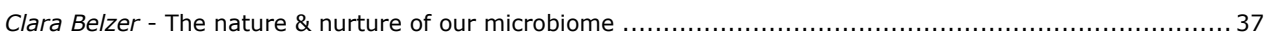

Detmer Sipkema- The impact of depth: sponge-associated microbes and antimicrobial activity change ...........38

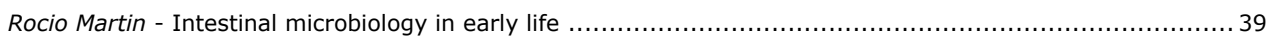

Ivonne Nijenhuis - Exploring anaerobic microbial dehalogenation reactions applying compound-specific

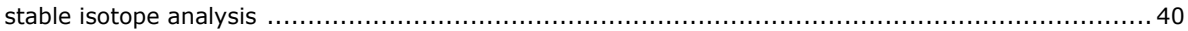

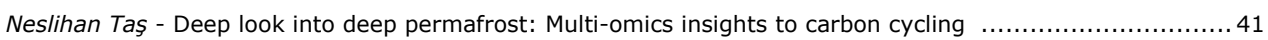

Johanna Gutleben - Integrating (meta)genomics data into sponge symbiont cultivation .........................42

Oral presentations - Microbial physiology - October 19, 2017 ............................. 43

Bernhard Schink - 100 years of microbiology in Wageningen and 50 years of syntrophy: What did we learn ...44

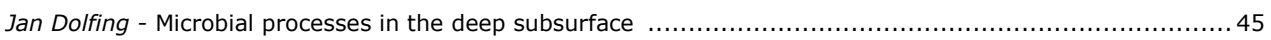

Diana Sousa - Anaerobic microbial growth on one-carbon compounds ......................................... 46

Wakako Ohtsubo - Exploration of sulfate-reducing bacteria colonizing the intestinal tracts .....................47

Jo Philips - Isolation and characterization of novel electrotrophic microorganisms using metallic iron

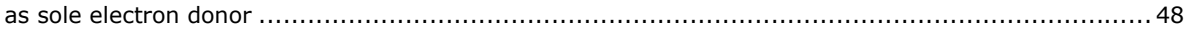

Laura C. Valk - Galacturonate fermentation by anaerobic chemostat enrichment cultures.......................49

Wei Du - Harnessing microbial evolvability towards developing stable photosynthetic cell factories..............50

Alette Langenhoff - Pharmaceutical biodegradation under three anaerobic redox conditions evaluated by

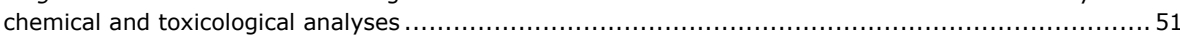

Oral presentations - Bacterial genetics - October 20, $2017 \ldots \ldots \ldots \ldots \ldots \ldots \ldots \ldots \ldots \ldots \ldots \ldots \ldots$

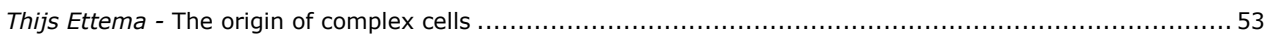

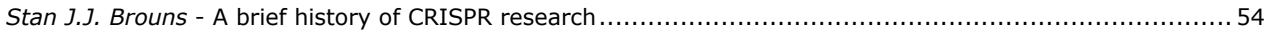

Elleke F. Bosma - Bacillus smithii as a novel thermophilic cell factory: from isolation to engineering ............. 55

Melvin Siliakus - Converting Escherichia coli into an "Archaebacterium" with a hybrid hetrochiral membrane.. 56 


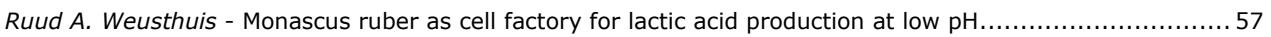

Joyce H.G. Lebbink - Programmable CRISPR-CAS9 roadblocks to study mechanisms in DNA mismatch repair

Mike Jetten - 100 years of "impossible" anaerobic microbiology and beyond ................................... 59

Poster pitches - October 19, 2017 - 12.00-12.10 hrs...................................... 60

Siavash Atashgahi - A benzene-degrading nitrate-reducing microbial consortium displays aerobic and

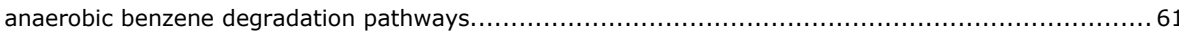

Loo Wee Chiao - Metatranscriptomics study of trophic interaction between Akkermansia muciniphila and butyrogenic gut commensals

Jens Christian Claussen - Cyclic coevolutionary interactions, coexistence fixed point and biodiversity control: an examplaric minimal model of mutualisms and feedback

Sharon Geerlings - Isolation of bacteriophages against Akkermansia muciniphila .....

Ioannis Kostopoulos - Mucolytic activity of Akkermansia muciniphila enables human milk oligosaccharides degradation. .....

Noora Ottman - The living environment shapes intestinal microbial communities and immune responses in a mouse model

Jules L. Rombouts - Microbial diversity in xylose or glucose fermenting enrichments: the impact of continuous growth limitation.....

Sudarshan A. Shetty - Human intestinal core microbiome: clues for a minimal microbiome .....................6 68

Alexander Umanets - Characterisation of small and large intestinal microbial composition in wild boars .........69

Hugo de Vries - The host-microbiota interactome in early life and its role in gut immune development ..........70

Prarthana Mohanraju - Autonomous processing of a single crispr-rna array by CRISPR-CAS12a enables

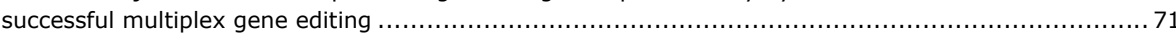

Poster pitches - October 19, 2017 - 16.25-16.35 hrs........................................ 72

Monir Mollaei - Syntrophic cocultures of geobacter sulfurreducens and syntrophobacter fumaroxidans growing on propionate and fe (iii) or a solid electrode as electron acceptors ........................... 73

Marjet J. Oosterkamp - Identification of arsenic-oxidizing bacteria in rapid sand filters .......................... 74

Martijn Diender - Microbial systems for conversion of syngas to bio-based products........................... 75

Roy Geerts - Assessment of ready biodegradability of alkyl polyglucosides using pure and mixed

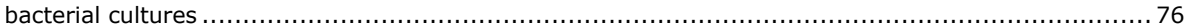

Yuan Feng - Enrichment of sulfidogenic bacteria from the human intestinal tract ............................. 77

Susakul Palakawong Na Ayudthaya - Organic acid production from starchy waste by rumen derived microbial communities..................

Peer H.A. Timmers - Syntrophic acetate oxidation at haloalkaline conditions.................................. 79

Daan M. van Vliet - Enrichment and isolation of novel polysaccharide-degrading anaerobes from abyssal

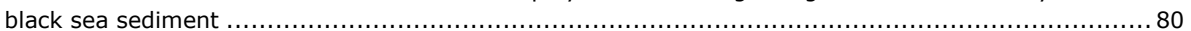

Nikolaos Strepis - Trichococcus species as catalysts for biotechnological production of 1,3-propanediol .........81

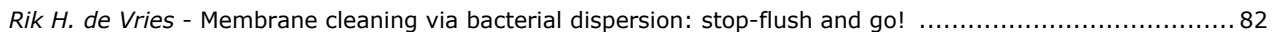

Poster presentations - Microbial ecology .................................................... 83

Nora B. Sutton - Relating microbial community to in situ natural attenuation of micropollutants.................. 84 Mirza Nabeel Baig - Biological Control of Different Fungal Pathogens by Ganoderma lucidum ...................... 85 Matthew Callaghan - Genetic and phenotyic variation between two phylogenetic distinct clusters of

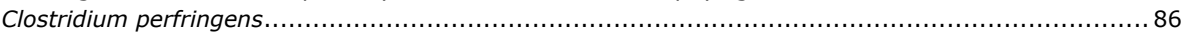
Jens Christian Claussen - Boolean analysis reveals systematic interactions among low-abundance

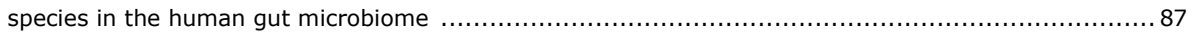
Ton T.H. Dat - Diversity of sponge-associated bacteria cultured to date and influence of culture conditions .... 88 Janneke Elzinga - Enzymatic degradation of human milk oligosaccharides by Akkermansia muciniphila .........89 
Asimenia Gavriilidou - 'Genome' exploration of members of the flavobacteriaceae family isolated from different sponge species: a comparative genomics approach

Anak Agung Gede Indraningrat - Depth matters: composition and antimicrobial activity of microbial communities associated with Xestospongia muta and Agelas sp. along a depth gradient .....

Hikmah Z. Hassan - Molecular analysis of microbial community dynamics during in vitro fermentation of fibres derived from three indonesian local tubers by a human fecal inoculum .....

Gerben D.A. Hermes - Acetate as metabolic target in the prevention of overweight-related chronic metabolic disorders .

Jie Lian- Screening algal-bacterial interations for new molecules ................................................ 94

Peng Peng - Vitamin $\mathrm{B}_{12}$ mediated dechlorination in pristine hypersaline lakes ................................ 95

Ran An - The effects of synbiotic supplementation on small intestine and fecal microbiota .......................96

Shanti Ratnakomal - Screening for antimicrobial activity of marine actinobacteria from lembeh strait,

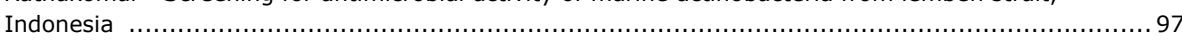

Carrie Wegh - The effect of fiber and prebiotics on children's gastrointestinal disorders and microbiome ........98

Caifang Wen - The potential of prebiotic effect on microbiota colonization, gut function and animal

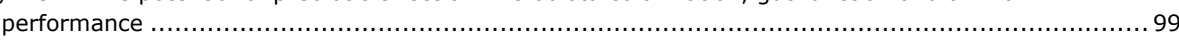

Ying Zheng - Release, degradation and detection of phenylpropionate during anaerobic degradation of rice straw

Romy D. Zwittink - Association between duration of intravenous antibiotic administration and early life microbiota development in late preterm infants....

Poster presentations - Microbial physiology

Nohemi Campos-Quevedo - Sulfate-reducing consortia consuming substrate at acidic conditions

Leonor Guedes Da Silva - Grow or store? exploring metabolic decision making under feast/famine conditions using dynamic $13 \mathrm{C}$ flux analysis....

Lot van der Graaf - Acidothermophilic bioreduction of sulfur for the recovery of valuable metals .............. 105

Jueeli D. Vaidya - Propionibacterium sp. strain JV5: a novel propionate producer isolated from ruminal

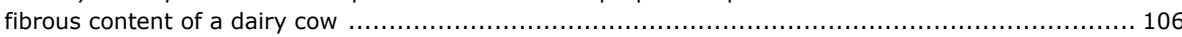

Poster presentations - Bacterial genetics

Christos Batianis - Model-driven design of novel Pseudomonas putida strains

Stamatios Damalas - The MetaBrick platform for DNA manipulation and standardization. Bridging Synthetic Biology standards for optimized interoperability

Pauline Folch - Modifications of the pyruvate dehydrogenase complex of E. coli and their effect on enzyme activity and growth.

Joyshree Ganguly - Genetic tool development and sporulation mechanisms in thermophilic clostridia........... 111

Jeroen G. Koendjbiharie - Metabolic engineering of thermophilic Clostridia for production of dicarboxylic acids.

Ioannis Mougiakos - Characterizing a thermostable Cas9 and exploring its potential as a prokaryotic genome engineering tool

Thijs Nieuwkoop - Improving heterologous protein production by tweaking transcript elements ............... 114

Teunke van Rossum - A transcriptional regulator-based selection system for obtaining novel biocatalysts .... 115

Yifan Zhu - A non-canonical seed sequence regulates targeting by the CRISPR/CMR complex...... 


\section{General information}

\section{Abstracts}

This book contains the abstracts of oral presentations followed by the poster pitch abstracts and poster abstract. The organizing committee does not take any responsibility for scientific of typographical errors.

\section{Oral presentations}

All oral presentations will be held in the Waaierzaal (room 1040, $1^{\text {st }}$ Floor Orion Building).

\section{Posters}

The posters are arranged per session in alphabetical order of the first authors' last names. They will be on display throughout the symposium and can be found in the Spot (restaurant Orion, ground floor). Poster sessions are held on $19^{\text {th }}$ October 2017.

\section{Coffee/tea/drinks and lunches}

During the symposium all coffee/thee/drinks and lunches will be served in restaurant the Spot.

\section{Symposium dinner, Thursday October 19, 2017}

All participants of the Microbiology centennial symposium are cordially invited.

At 19.00 hours the Conference dinner starts at Hotel-Restaurant De Wageningsche Berg HotelRestaurant De Wageningsche Berg is beautifully situated on the Wageningen Berg and offers a view over the river, the floodplains and the Betuwe. Enjoy the natural beauty from the panoramic terrace. We offer an buffet style dinner with drinks, dessert and coffee.

Hotel-Restaurant De Wageningsche Berg, Generaal Foulkesweg 96, 6703 DS Wageningen.

www.hoteldewageningscheberg.nl

Dinner is included in the standard Conference fee.

\section{Bus service}

Bus service is provided on Thursday, October 19, 2017 between 17.30-19.00 hrs between the Orion Building - Hotel-restaurant De Wageningsche Berg v.v.

Departure of the busses is in front of Orion Building.

Note that there is no bus service after the Conference dinner.

\section{WiFi at Orion}

There is free WiFi available during the Conference. The WiFi codes are available at the registration desk and are strictly for Microbiology centennial symposium - participants. Please be aware that the network regulations for working on the Wageningen University \& Research network are applicable. If anyone using the MIB100 WiFi-codes causes security issues on the Wageningen University \& Research network unfortunately the WiFi option will be closed. 


\section{Sponsors}

The Organising Committee gratefully acknowledges the support of:

The Soehngen Institue of Anaerobic Microbiology (SIAM)

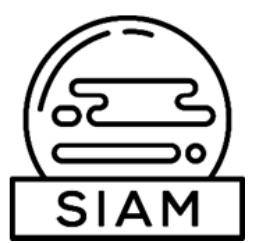

Wageningen University \& Research Vlag Graduate School

Wageningen University \& Research WIMEK Research Institute
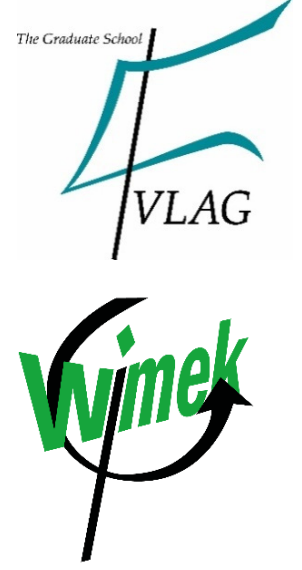

NOW-Spinoza

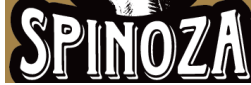




\section{Organising Committee}

Wageningen University \& Research, The Netherlands

- Willem M. de Vos

- Anja van Holland

- Desiree Meijer

- Hedy Wessels

\section{Conference Secretariat}

Wageningen University \& Research, The Netherlands

- Hedy Wessels

\section{Scientific Committee}

Wageningen University \& Research, The Netherlands

- Servé Kengen

- John van der Oost

- Hauke Smidt

- Alfons Stams

- Willem M. de Vos 
Programme 


\section{Microbiology Centennial Symposium}

\section{October 18-20, 2017, Orion, Wageningen Campus}

Registrationdesk: Lobby, Orion Building (Bronland 1, Building 103, 6708 WH Wageningen)

All breaks: $\quad$ Restaurant 'The Spot', Orion building

All sessions: $\quad$ Plenary meeting room 1040, Waaierzaal Orion building

Poster sessions: Restaurant 'The Spot', Orion building

\section{Wednesday, October 18, 2017}

16.00 hrs

$17.00-19.00 \mathrm{hrs}$

$17.30 \mathrm{hrs}$

$18.15 \mathrm{hrs}$

19.00-20.30 hrs
Registration with coffee/tea

Opening Session - Wageningen School of Microbiology

ft Alex Zehnder, Zurich- Edmonton \&

Willem $M$ de Vos, Wageningen-Helsinki

Key Note - Jan Roelof van der Meer, Lausanne $\mathrm{CH}$ What Can We Learn From Microbes in the Environment

Key Note - Mark van Loosdrecht, Delft NL At the Interface of Microbiology

Drinks \& Bites 


\section{Thursday, October 19, 2017}

$08.30 \mathrm{hrs}$

09.00-12.15 hrs

$09.05 \mathrm{hrs}$

$09.45 \mathrm{hrs}$

10:05 hrs

$10: 25-10.55 \mathrm{hrs}$

$10: 55$ hrs

$11: 15$ hrs

$11: 35 \mathrm{hrs}$

$11: 50 \mathrm{hrs}$

$12: 00 \mathrm{hrs}$
Registration

Microbial Ecology - ft Hauke Smidt \&

Clara Belzer Wageningen \& Nanjing

Key Note - Hauke Harms, Leipzig DE

Domesticating Complex Microbial Ecosystems

Clara Belzer, Wageningen, NL

The nature \& nurture of our microbiome

Detmer Sipkema, Wageningen, NL

The impact of depth: sponge-associated microbes and antimicrobial activity change

Coffee Break

Rocio Martin, Singapore, SP

Intestinal microbiology in early life

Ivonne Nijenhuis, Leipzig, DE

Exploring anaerobic microbial dehalogenation reactions applying compound-specific stable isotope analysis.

Neslihan Tas, Berkeley, USA

Deep look into deep permafrost: Multi-omics insights to carbon cycling

Johanna Gutleben, Wageningen, NL

Integrating (meta)genomics data into sponge symbiont cultivation

10 Poster Pitches (1 minute each)

Siavash Atashgahi (Wageningen, NL) A benzene-degrading nitratereducing microbial consortium displays aerobic and anaerobic benzene degradation pathways

LooWee Chia (Wageningen, NL) Metatranscriptomics study of trophic interaction between Akkermansia muciniphila and butyrogenic gut commensals

Jens Christian Claussen (Bremen, DE) Cyclic coevolutionary interactions, coexistence fixed point and biodiversity control: An exemplaric minimal model of mutualisms and feedback

Sharon Geerlings (Wageningen, NL) Isolation of bacteriophages against Akkermansia muciniphila

Ioannis Kostopoulos (Wageningen, NL) Mucolytic activity of Akkermansia muciniphila enables human milk oligosaccharides degradation 
$12.15-13.30 \mathrm{hrs}$

$13.30-16.45$ hrs

$13.35 \mathrm{hrs}$

14.15 hrs

$14.40 \mathrm{hrs}$

$15.05 \mathrm{hrs}$

$15.35 \mathrm{hrs}$

15.45 hrs

15.55 hrs

16.05 hrs

16.15 hrs
Noora Ottman (Stockholm, Sweden) The living environment shapes intestinal microbial communities and immune responses in a mouse model

Jules Rombouts (Delft, NL) Microbial diversity in xylose or glucose fermenting enrichments: The impact of continuous growth limitation

Sudarshan Shetty (Wageningen, NL) Human intestinal core microbiome: clues for a minimal microbiome

Alexander Umanets (Wageningen, NL) Characterization of small and large intestinal microbial composition in wild boars

Hugo de Vries (Wageningen, NL) The host-microbiota interactome in early life and its role in gut immune development

Prarthana Mohanraju (Wageningen, NL) Autonomous processing of a single CRISPR-RNA array by CRISPR-Cas12a enables successful multiplex gene editing

Lunch \& Poster Session

Microbial Physiology - ft Fons Stams \& Diana Sousa, Wageningen-Braga

Key Note - Bernhard Schink, Konstanz, DE

100 Years of Microbiology in Wageningen and 50 Years of Syntrophy: What Did We Learn?

Jan Dolfing, Newcastle, UK

Microbial processes in the deep subsurface

Diana Sousa, Wageningen, NL

Anaerobic microbial growth on one-carbon compounds

Coffee Break

Wakako Ohtsubo, Tohoku, JP

Exploration of sulfate-reducing bacteria colonizing the intestinal tracts

Jo Philips, Gent, BE

Isolation and characterization of novel electrotrophic microorganisms using metallic iron as sole electron donor

Laura Valk, Delft, NL

Galacturonate fermentation by anaerobic chemostat enrichment cultures

Wei Du, Amsterdam, NL

Harnessing microbial evolvability towards developing stable photosynthetic cell factories

Alette Langenhoff, Wageningen, NL

Pharmaceutical biodegradation under three anaerobic redox conditions evaluated by chemical and toxicological analyses 
Monir Mollaei (Leeuwarden, NL) Syntrophic cocultures of Geobacter sulfurreducens and Syntrophobacter fumaroxidans growing on propionate and Fe (III) or a solid electrode as electron acceptors

Marjet Oosterkamp (Delft, NL) Identification of arsenic-oxidizing bacteria in rapid sand filters

Martijn Diender (Wageningen, NL) Microbial systems for conversion of syngas to bio-based products

Roy Geerts (Deventer, NL) Assessment of ready biodegradability of alkyl polyglucosides using pure and mixed bacterial cultures.

Yuan Feng (Wageningen, NL) Enrichment of sulfidogenic bacteria from the human intestinal tract

Susakul Palakawong (Wageningen, NL) Organic acid production from starchy waste by rumen derived microbial communities

Peer Timmers (Leeuwarden, NL) Syntrophic acetate oxidation at haloalkaline conditions

Daan van Vliet (Wageningen, NL) Enrichment and isolation of novel polysaccharide-degrading anaerobes from abyssal black sea sediment

Nikoloas Strepis (Wageningen, NL) Trichococcus species as catalysts for biotechnological production of 1,3-propanediol

Rik de Vries (Leeuwarden, NL) Membrane cleaning via bacterial dispersion: stop-flush and go!

$16.45-18.00 \mathrm{hrs}$

$19.00-23.00 \mathrm{hrs}$
Drinks \& poster session

Dinner @ Wageningsche Berg 


\section{Friday, October 20, 2017}

$09.00-12.00 \mathrm{hrs}$

09.05 hrs

09.45 hrs

$10.10 \mathrm{hrs}$

$10.30 \mathrm{hrs}$

$11.00 \mathrm{hrs}$

11.20 hrs

$11.40 \mathrm{hrs}$

$12.00 \mathrm{hrs}$

$12.10 \mathrm{hrs}$

$13.00 \mathrm{hrs}$

13.30 hrs

$14.00 \mathrm{hrs}$
Bacterial Genetics ft John van der Oost \& Stan Brouns Wageningen Montana-Delft

Key Note - Thijs Ettema, Uppsala, SE The Origin of Complex Cells

Stan Brouns, Delft, NL

A brief history of CRISPR research

Elleke Bosma, Copenhagen, DK

Bacillus smithii as a Novel Thermophilic Cell Factory: From Isolation to Engineering

Coffee Break

Melvin Siliakus, Texel, NL

Converting Escherichia coli into a "archaebacterium" with a hybrid heterochiral membrane

Ruud Weusthuis, Wageningen, NL

Monascus ruber as cell factory for lactic acid production at low $\mathrm{pH}$

Joyce Lebbink, Rotterdam, NL

Programmable CRISPR-Cas9 roadblocks to study mechanisms in DNA mismatch repair

Bauke Oudega, Amsterdam, NL Mighty Microbes

Outlook \& Closing Session ft Willem M. de Vos, Wageningen, Helsinki

Key Note - Mike Jetten, Nijmegen NL

100 Years of "Impossible" Anaerobic Microbiology and Beyond

Lunch \& Departures

End of Symposium

SIAM Meeting (see SIAM programme, only for invited participants) 


\section{Brief introduction Keynote speakers}




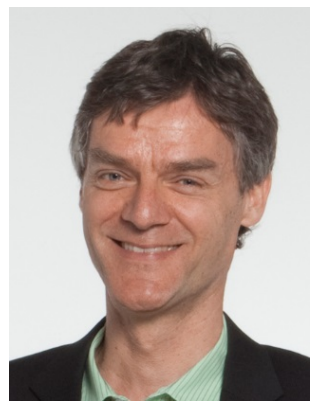

Jan Roelof van der Meer (1961) studied Environmental Sciences at Wageningen University and obtained his $\mathrm{PhD}$ in Environmental Microbiology from the same university, under supervision of Prof. Alexander Zehnder and Prof. Willem de Vos. He worked as a postdoc at the Netherlands Institute for Dairy Research before moving to the Swiss Federal Institute of Aquatic Sciences (Eawag) as Junior Group Leader in 1992. Since 2003 he is Professor in Environmental Microbiology at the University of Lausanne, and in 2011 he became Director of the Department of Fundamental Microbiology. His research focuses on understanding the mechanisms of genetic adaptation to toxic compounds in bacteria, development of synthetic gene circuits for and applications of bacterial biosensors, and microbial community engineering. He supervised $35 \mathrm{PhD}$ students and coordinated several large national and international research projects. His group published 170 peer-reviewed articles (citation indices: 43 and 48). For his work on bacterial biosensors he was awarded the Erwin Schrödinger Prize of the Helmholtz Foundation. As a teacher he received the Excellence in Teaching Biology award. 


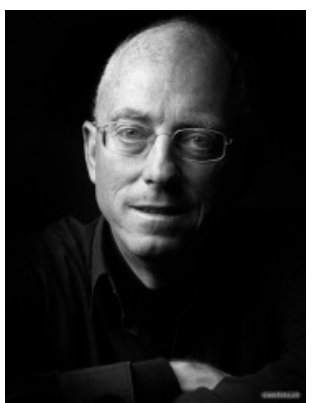

Mark van Loosdrecht is Professor of Environmental Biotechnology at Delft University of Technology, The Netherlands. He graduated (MSc and PhD) at Wageningen University with specialisation in Microbiology and Physical and Colloid Chemistry. He went to TU Delft in 1988 and became Full Professor in 1998. His research is characterized by the combination of scientific understanding of complex systems and development of new processes. His scientific interests are related to biofilm processes, nutrient conversion processes and the role of storage polymers in microbial ecology. In particular, he is interested in new processes related to wastewater treatment and resource recovery. His research has resulted in several processes currently applied on full scale such as the Sharon process, Anammox process and Nereda process. Currently processes for bioplastics and hydrogels production from waste are in the scale-up phase. He is active member of the International Water Association (IWA) and past chairman of the Biofilm and the Nutrient removal specialist groups. He is Editor-in-Chief of Water Research. He obtained several prizes for his work, including the Lee Kuan Yew Singapore Water Prize, the NWO Spinoza Award, the STW Simon Stevin award and the IWA Grand Award. He is member of the Royal Dutch Academy of Arts and Sciences (KNAW), the Dutch Academy of Engineering (AcTI) and the US National Academy of Engineering (NAE). He was awarded a knighthood in the order of the Dutch Lion. He has published over 650 scientific papers (google scholar h-index 120), holds around 20 patents and has supervised over 50 PhD students. 


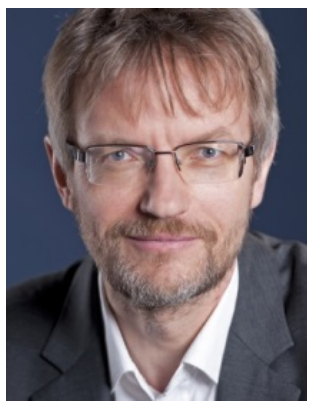

Hauke Harms (1961) studied Biology at the University of Hamburg, where in 1990 he received his $\mathrm{PhD}$ for a study about microbial dioxin degradation. From 1991 to 1993 he worked as a long-term fellow of the European Environmental Research Organization (EERO) with Prof. Alexander Zehnder at the Laboratory of Microbiology in Wageningen. Here and during his subsequent career stage as a Research Associate at the Swiss Federal Institute of Environmental Science and Technology (EAWAG) he dealt with the physico-chemical constraints of pollutant degradation in the terrestrial environment. In 1998, Hauke Harms became Assistant Professor of soil microbiology at the Swiss Federal Institute of Technology Lausanne (EPFL) and in 2004, he moved to his present positions as Department Head at the Helmholtz Centre for Environmental Research - UFZ (Leipzig, Germany) and full Professor for Environmental Microbiology at the University of Leipzig. Besides being responsible for a broad range of ecological, microbiological and biotechnological studies conducted by 10 independent research groups in his department, Hauke Harms is Head of the Environmental Engineering and Biotechnology section (equivalent to a dean) at his research centre and acts as the coordinator of the Helmholtz research program 'Terrestrial Environment'.

Hauke Harms has (co-)authored 250 peer-reviewed publications with an H-factor (ISI) of 46. In 2010, he was awarded the prestigious Schrödinger-Prize for interdisciplinary research. 


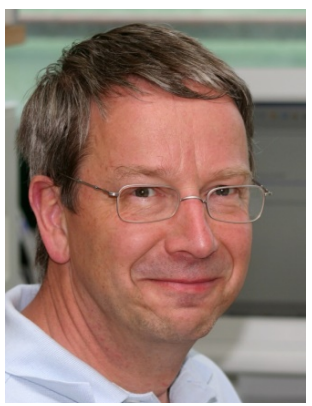

Bernhard Schink (1950) studied biology with special emphasis on microbiology at Marburg and Göttingen and obtained his doctoral degree in Göttingen in 1977. After postdoc stays in Madison, Wisconsin, USA, and Konstanz, he became professor in Marburg and Tübingen before he finally settled again in Konstanz where he holds a chair for limnic microbiology. His main research interest is on the physiology, ecology and biochemistry of anaerobic microbial transformation activities in natural and manmade ecosystems. He is a member of the German National Academy of Sciences Leopoldina, the Academy of Sciences in Literature in Mainz as well as the American and the European Academy of Microbiology, and he was vice president and president of FEMS. He supervised more than 50 doctoral candidates in his group and published more than 300 ISI-listed articles in peer-reviewed journals (h-index 56). He is an editor and a member of editorial boards of several microbiological journals. 


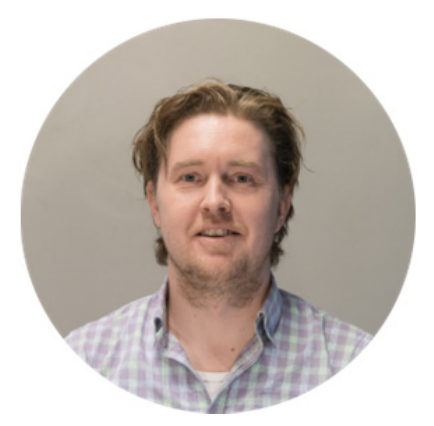

Thijs J. G. Ettema (1977) is an evolutionary microbiologist at Uppsala University (Sweden) where he heads a group that focuses on the exploration of microbial diversity with nextgeneration genomics approaches. He received his PhD at the Department of Microbiology at Wageningen University (The Netherlands) in 2005. After a brief postdoctoral stay at the Radboud University in Nijmegen (The Netherlands), he moved to Uppsala University (Sweden) in 2006, where he currently holds an associate professorship at the Department of Cell and Molecular Biology. His group has a broad research focus and works on a variety of scientific questions connected to microbial diversity and evolution that cover all three Domains of Life, as well as viruses. One overarching theme in his research involves the origin of complex cells types (eukaryotes). Recently, he discovered a new group of archaea, the ASGARD archaea, providing new, compelling evidence that complex cellular life evolved from an archaeal ancestor that was more advanced than was presumed before. He is currently heading the the Microbial Single Cell Genomics facility at the SciLifeLab, a leading Life Sciences institute in Sweden. Thijs Ettema is the recipient of the 2007 Kluyver award by the Royal Dutch Society for Microbiology, he was named 'Future Research Leader' by the Swedish Foundation for Strategic Research in 2012, and was elected EMBO Young Investigator by the European Molecular Biology Organization in 2016. His research is, amongst others, funded by an ERC Starting Grant. 


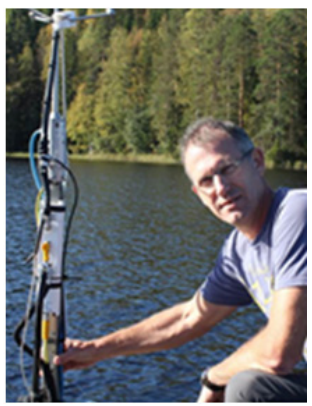

Mike Jetten (1962) is a world leader in the field of environmental microbiology. He did his PhD (1991) at WUR on aceticlastic methanogens, followed by a post doc at MIT on metabolic engineering of amino acid producers. From 1994-2000 he was assistant professor at TU Delft on Nitrogen cycle microbiology. Since 2000 he is chair of the Department of Microbiology at Radboud University, Nijmegen. He received the prestigious ERC Advanced Grant in 2008 for his research on anammox bacteria, the Spinozapremie in 2012, and a second ERC Advanced grant in 2013 to study the ecology of methane oxidizers. Since 2013 he scientific director of the Gravitation consortium on anaerobic microbiology (Soehngen Institute of anaerobic microbiology). He is a member of the Royal Netherlands Academy of Sciences, the European Academy of Science and EMBO. In 2013 he was bestowed with a knighthood for his exceptional services to science. He has published more than 400 papers that have been cited more than 38000 times. His $\mathrm{H}$ index is 95 . He has supervised $35 \mathrm{PhD}$ theses and 32 post docs, and $22 \mathrm{PhD}$ students and 8 post docs are currently working in his laboratory. In September 2015, his team started the first Microbiology master education in the Netherlands. $\mathrm{He}$ has been invited more than 250 times for keynote lectures and organized many international meetings and conferences. He holds several patents. 


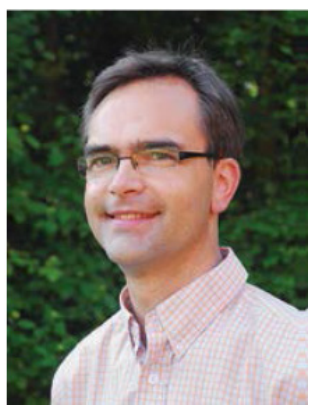

Hauke Smidt (1967) studied Biotechnology at the Technical University of Braunschweig, University of Kyoto, Japan, and University of Stuttgart, Germany. He received his PhD in 2001 at Wageningen University, and has worked as a postdoc with Prof. Dave Stahl at the University of Washington, Seattle, focusing on the development of DNA arrays for microbial community analyses. Since 2003, he has been leader of the Molecular Ecology research group within the Laboratory of Microbiology at Wageningen University. Key questions are addressed within national and international multi-investigator consortia, and include i) Microbial communities in environmental biotechnology, ii) Functionality of microbiota associated with body surfaces (gut, skin, mouth) in humans, production animals, as well as animal (mostly rodent) models, and iii) Microbes and their cellular biomarkers as proxies for ecosystem life history \& environmental change. Research approaches include innovative cultivation-based as well as molecular approaches that aim at understanding and predicting ecosystem structure and function. Hauke Smidt has recently coordinated the EU-FP7 funded project INTERPLAY focusing on the interplay between intestinal microbiota and intestinal development and health in pigs, is member of the Management Team of the National BE-Basic program, and has been Senior Scientist and Theme Council member at TI Food \& Nutrition.

Hauke Smidt has (co-)authored over 200 peer-reviewed publications, with an ISI WoS H-factor of currently 45 . He is (co-)inventor of several patents, and is member of the Editorial Board of several journals, including PlosOne, PeerJ and Microbial Ecology. In 2008, Hauke Smidt has been appointed Visiting Professor at Nanjing Agricultural University, and since 2010, he holds a Personal Chair in "Complex Microbial Ecosystems" at Wageningen University. 


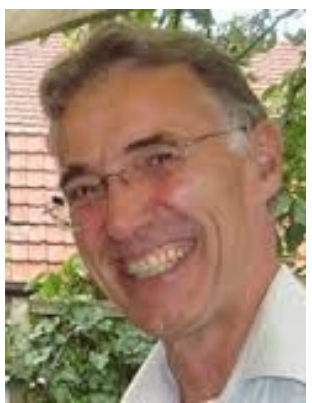

Alfons J.M. Stams (1953) studied Molecular Sciences at Wageningen University and obtained his PhD at Groningen University. He studies the physiology and ecology of anaerobic microorganisms that play an important role in environmental biotechnological processes, such as wastewater treatment, soil remediation and the production and recovery of biofuels and organic and inorganic chemicals. He obtained an early career fellow of the Netherlands Royal Academy of Arts and Sciences (KNAW) and received a NWO Chemical Sciences TOP grant, an advanced ERC grant and a Gravitation grant of the Netherlands Ministry of Education, Culture and Science. He has (co)supervised over $50 \mathrm{PhD}$ students and published more than 400 articles in peer-reviewed journals and books. His current ISI $\mathrm{H}$-index is 57 (Google Scholar 75). He is editor of Applied and Environmental Microbiology and of FEMS Microbiology Ecology. 


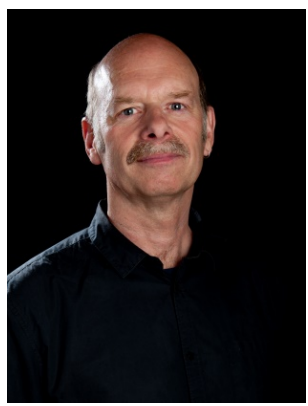

John van der Oost (1958) studied Biology and obtained a PhD at the Free University in Amsterdam. He spent $>3$ years abroad, 2.5 years of which at the European Molecular Biology Laboratory in Heidelberg. He returned to Amsterdam with a 3-year fellowship of the Royal Netherlands Academy of Sciences and Arts (KNAW). In 1995, he started an independent research group on Bacterial Genetics within the Laboratory of Microbiology at Wageningen University. In 2005 he was appointed Full Professor at Wageningen University, with a personal chair in Molecular Microbiology and Biochemistry, and since 2009 he is visiting professor at the Montana State University (Bozeman, USA). He has supervised over 50 PhD students and with these and other collaborators he published over 260 peer-reviewed publications. He is an inventor of over 20 patents or patent applications and has an ISI h-index 55 (Google Scholar 61). He is laureate od a NWO VICI grant, and he has obtained two NWO TOP grants. He has been coordinator of an EU project and a Marie Curie Research \& Training Network. He has been elected as member of the European Molecular Biology Organization (EMBO). John's research aims to understand and exploit microbial control and defence systems. His current interest is focused on developing tools for enhancing the performance of enzymes and bacterial cell factories, and on discovering and applying guided nucleases such as CRISPR-Cas and Argonaute. 


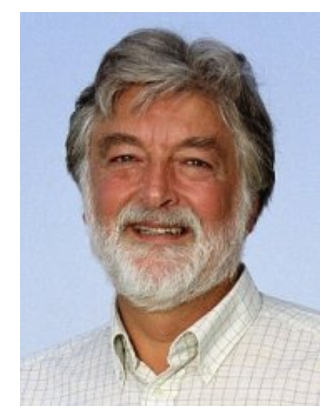

Alexander Zehnder (72) studied Natural Sciences at ETH-Zurich and did his PhD in Microbiology at the Swiss Federal Institute for Aquatic Sciences (EAWAG) and ETH Zurich. His career brought him as a Postdoc to Madison, Wisconsin (USA) and as an Assistant Professor to Stanford University, California. From 1982 to 1992 he served as Professor for Microbiology at Wageningen University and from 1992 to 2004 as Professor for Environmental Biotechnology at ETH-Zurich and Director of EAWAG. From 2004 to 2008, he was President of the ETH Domain. Since 2008 he is Director of Water Resources at Alberta Innovates and since 2009 also Visiting Professor at Nanyang Technological University (NTU) in Singapore. He supervised over $80 \mathrm{PhD}$ students and published with them and his collaborators over 250 papers. He has an honorary doctoral degree from the University Henri-Poincaré, Nancy, France, is a member of the Netherlands Royal Academy of Arts and Sciences (KNAW), of the Swiss Academy of Engineering Science (SATW) and Foreign Member of the Russian Academy of Science (NAUK). He got the Order of the Federal Republic of Germany (Bundesverdienstkreuz), 1st Class. He gave the inaugural Perry McCarty Distinguished Lecture, Stanford University, California, the Charles and Mary O'Melia Lecture in Environmental Sciences, Johns Hopkins University, Baltimore and the Dr. John W. Macgregor Memorial Lecture, University of Alberta, Edmonton, Canada. He received a number of awards, chairs the Scientific Advisory Board of SCELSE, one of Singapore's Research Centers of Excellence, and is on the Board of Trustees for the Knowledge Award of Mohammed bin Rashid al Maktoum Foundation. 


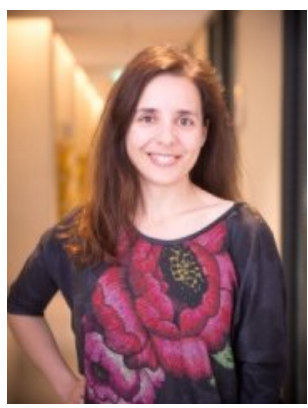

Diana Sousa is a tenure track Assistant Professor at the Laboratory of Microbiology from Wageningen University. She studied Biological Engineer at the University of Minho and obtained her PhD from the same university in 2007. During her PhD she specialized on the microbiology of the conversion of long-chain fatty acids (LCFA) in anaerobic reactors and, inherently, on obligate syntrophic microorganisms. From 2007 to 2013 she worked as Assistant Professor at the Department of Biological Engineering from the University of Minho. During this type she supervised $4 \mathrm{PhD}$ students and was responsible for two scientific projects, one on the microbial conversion of LCFA and the other on the bioconversion of syngas. In May 2013 she moved to the Laboratory of Microbiology from Wageningen University where she continues her research on these topics. She has published 49 articles and book chapters and has been invited for several keynote lectures in international conferences. Her special interest lays on the study of the metabolic pathways, microbes and (synthetic) microbial networks that convert C1 molecules such as carbon monoxide, carbon dioxide, methane and methanol, and their application to produce high-value chemical building blocks. 


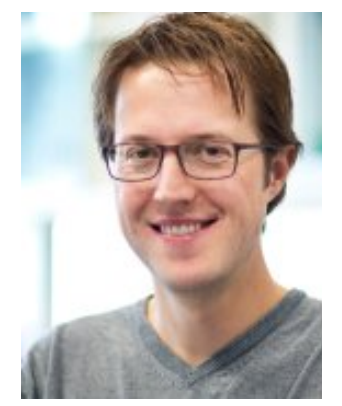

Stan J.J. Brouns (1978) is a Molecular Microbiologist who studied Molecular Life Sciences and graduated from Wageningen University in the Netherlands in 2001. He received a PhD in Microbiology in 2007 at the same University after studying the sugar metabolism of hyperthermophilic archaea. From 2006 he has been interested in elucidating the mechanism of CRISPR-mediated bacteriophage defense in prokaryotes. In 2011 he started his own group at the Laboratory of Microbiology (Wageningen University) studying microbe-bacteriophage relationships after obtaining national (VIDI) and European funding (ERC). Dr. Brouns obtained a tenured Associate Professor position at the department of Bionanoscience (Kavli Institute of Nanoscience) of Delft University of Technology and is still affiliated with Wageningen University. He has contributed to over 56 scientific publications, has an h-index of 30 , and has received a number of awards for his pioneering CRISPR work. His lab is interested in the interaction between microbes and viruses and studies the mechanisms that bacteria and archaea use to protect themselves from virus infection including CRISPR. His lab explores the adaptations that viruses have evolved to avoid defense systems and engineers bacteriophages for phage therapy applications. 


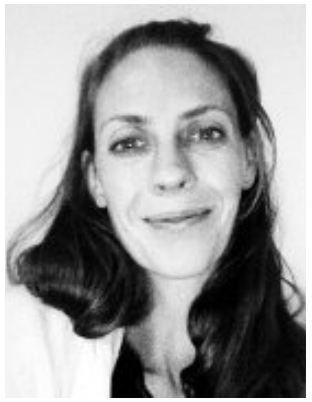

Clara Belzer is Assistant Professor at the Laboratory of Microbiology at Wageningen University and Research (WUR). Her research aims to understand the way microbial species in the gastrointestinal tract are able to degrade host-produced glycans (human milk and mucus). The ability of these microorganisms to ferment host-produced glycans makes them 'keystone species' within the intestinal microbiota that are crucial for immune, metabolic and neurologic imprinting. Topics vary from the microbiome of early and late life, human, mice, in health, and disease. The main microbial players are the mucus degrading Akkermansia muciniphila, intestinal butyrate producing Clostridia species and early life microbiota members like bifidobacteria.

Dr. Belzer was trained at the University of Utrecht (MSc.), Erasmus Medical Center (PhD.), and has worked at Harvard Medical School, TIFN and Wageningen University and Research. Dr. Belzer is currently part of NWO funded; Soehngen Institute of Microbiology (SIAM) and participates as a Dutch PI in the EU funded JPI-microbiomics consortium EARLYMICROHEALTH. Her research has lead to papers in top journals like Nature Medicine, PNAS and ISMEJ and has resulted in several patents. 


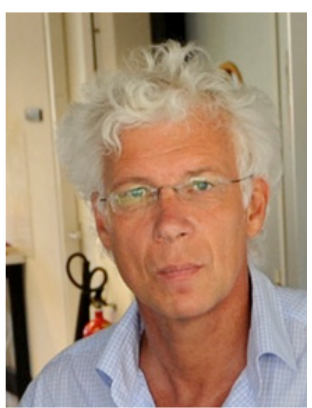

Willem M. de Vos (1954) studied Biochemistry and obtained a PhD at Groningen University, partly done at the Max Planck Institute for Molecular Genetics in Berlin. He is over 25 years Professor at Wageningen University, holds there the Chair of Microbiology, and serves as Professor of Human Microbiomics at the Medical Faculty of the University of Helsinki. He has supervised over $100 \mathrm{PhD}$ students and with these and other collaborators he published over 700 peer-reviewed publications. He is an inventor of over 40 patents or patent applications and has presently an ISI h-index $>100$ (Google Scholar $>125$ ). He is a member of the Netherlands Royal Academy of Arts and Sciences (KNAW), the European Academy of Microbiology, and various international Scientific Advisory Boards. He has received early career prizes for international PhD and post-doc stays, including EMBO, MPI, FEBS and CEC Fellowships, and various career awards, including the Miles Marschall Rhone-Poulenc International Dairy Science Award, the NWO Spinoza Award, and the Netherlands' Most Entrepreneurial Scientist Award. He also was elected as Finland Distinguished Professor, Finland Academy Professor of the Finnish Academy of Sciences, received an Honorary Doctorate in Medicine, and an ERC Advanced Grant. His research aims to understand and exploit microbes using molecular, (meta)genomics and systems approaches. His current interest is focused on the human intestinal tract microbiota and its relation with health and disease. 


\section{Opening session \\ October 18, 2017}




\title{
WHAT CAN WE LEARN FROM MICROBES IN THE ENVIRONMENT?
}

\author{
JAN ROELOF VAN DER MEER
}

University of Lausanne, Switzerland

Maybe this is the wrong title and I should say: 'What have I learned from microbes in the environment?'.

First: their enormous diversity. The thousands and thousands of different bacterial species in a gram of soil, in a milliliter of ocean or freshwater, in sediments and rocks; different again from one place to the next; what are they good for? Clearly, the approaches we learned as microbiologists 20-30 years ago are largely insufficient to understand the complexity and functioning of most microbial ecosystems.

Secondly, their astonishing capacity to adapt and evolve within short time-scales. In all ignorance and irresponsability, our societies have imposed linear economies of produceusage-waste. This has led to unprecedented selective conditions on ecosystems (e.g., toxic compounds, antibiotics), which have favored bacterial adaptation. Despite dire environmental consequences, these situations have enabled studying adaptation processes in great detail, and this has helped to appreciate the manifold ways existing in the microbial world to generate new genetic variants in extremely rapid manner. The details of some of those, which my laboratory has been studying, astound us to the present day.

Third lesson I have learned: the endless pool of interesting microbial capacities that find their origins in the environment and may be applied for a good cause. In my own research, we have attempted to utilize these capacities for developing processes that may help clean-up the environment.

What do I still wish to learn? How all these microbes live together in their seeming continuum of ever-changing communities. How we can understand their functioning and evolution, so that, perhaps at some future point, we can rationally redirect or manage them to ensure their stability, their functions to sustain plant and animal life, and that of ourselves. 


\section{MICROBIOLOGY AT THE INTERPHASE}

MARK VAN LOOSDRECHT (M.C.M.VANLOOSDRECHT@TUDELFT.NL)

Delft University of Technology

Outside laboratories most of microbial life occurs at surfaces. Understanding the behaviour of bacteria and organization of microbial communities is not only essential in better understanding of microbial ecology but also helps in designing of microbial based technology for water and wastewater treatment. Biofilm formation can only be understood when microbiology, chemistry and process engineering knowledge is adequately combined. The lecture will present an overview of understanding of microbial growth at interphases and how this can be used in order to design new processes for wastewater treatment and resource recovery. 
Oral presentations

Microbial ecology

October 19, 2017 


\title{
DOMESTICATING COMPLEX MICROBIAL ECOSYSTEMS
}

\author{
HAUKE HARMS
}

Helmholtz Centre for Environmental Research - UFZ, Germany

Microbial ecosystem services rely on the dynamic assembly and functional interplay of speciesrich microbial communities. The astounding functional robustness (stability, elasticity, resilience etc.) of natural communities driving biogeochemical cycles, degrading pollutants or supporting plant growth obviously originates from the fact that the right combination of active organisms is in place and, if needed, is dynamically backed-up via activation of formerly silent members or recruitment.

Our research attempts to understand the design-principles and mechanisms underlying functional robustness of natural microbial communities and to transfer this knowledge to processes in near-natural and intensely engineered technical settings. A strong focus is laid on the resilience of microbial community functions. Besides the biological composition of the involved communities we explicitly address structural features of the habitat as a driver of species richness, functional interplay and recovery after disturbance. Our research goes beyond bacterial communities and involves among others, structural functions of fungal networks and trophic interactions within communities.

The talk will give examples from our research that includes observations of functional communities in natural and technical systems, experimental approaches using constructed biodiversity and spatially explicit modelling of the dynamics of microbial ecosystem services. The talk will highlight the importance of subtle (and mostly opaque) helper functions of community members, the positive role of resource-sharing in communities, the effects of topdown control and the significance of the spatial extent and penetrability of the habitat for resilience. 


\title{
THE NATURE \& NURTURE OF OUR MICROBIOME
}

CLARA BELZER, PHD

\author{
Assistant Professor, Laboratory of Microbiology, \\ Wageningen University and Research
}

\begin{abstract}
The human body is colonized with numerous symbiotic microorganisms that play an important role in maintaining health. This so called 'microbiota' has the ability to release energy and nutrients from fibers that are indigestible to the host. The ability to breakdown these fibers lies with just a few among the hundreds of bacterial species that make up the human gut microbiota and this fermenting ability makes them 'keystone species' that serve a critical role in the structure and functioning of the intestinal microbial community. The other microbiota members depend on these 'keystone species' to release energy from the complex carbohydrate structures.
\end{abstract}

Apart from dietary fibers, the host itself produces structures rich in carbon and nitrogen such as mucus- and milk-oligosaccharides. These so-called glycans are not digested by the host itself. Instead several biological functions have been postulated, i.e., pre-biotic, anti-adhesive or anti-inflammatory effects. A small proportion of the microbiota members have co-evolved with their hosts by specializing in the consumption of host-produced glycans, such as mucusand milk-oligosaccharides. In turn, these microorganisms serve a beneficial and protective role for the host. The ability of these microorganisms to ferment host-produced glycans makes them 'keystone species' within the early life intestinal microbiota and mucus layer. Diverse members of this ecosystem metabolically depend on their glycan fermentation. Furthermore, colonization of these 'keystone species' early in life is crucial for immune and metabolic imprinting. In later life, permanent symbiotic colonization of the mucosal layer leads to continuous regulation of immune and metabolic processes and maintenance of host health. 


\title{
THE IMPACT OF DEPTH: SPONGE-ASSOCIATED MICROBES AND ANTIMICROBIAL ACTIVITY CHANGE
}

\author{
DETMER SIPKEMA ${ }^{1}$, ANAK AGUNG GEDE INDRANINGRAT ${ }^{1,2}$, GEORG STEINERT $^{1,5}$, \\ MICHAEL W. TAYLOR ${ }^{6}$, PETER J. SCHUPP ${ }^{5}$, LEONTINE E. BECKING ${ }^{3,4}$ AND HAUKE SMIDT ${ }^{1}$ \\ ${ }^{1}$ Laboratory of Microbiology, Wageningen University, The Netherlands \\ 2 Department of Biology, Faculty of Mathematics and Science Education, Institut Keguruan dan \\ Ilmu Pendidikan Persatuan Guru Republik Indonesia (IKIP PGRI) Bali, Indonesia \\ ${ }^{3}$ Marine Animal Ecology, Wageningen University, The Netherlands \\ ${ }^{4}$ Naturalis Biodiversity Center, Leiden, The Netherlands \\ ${ }^{5}$ Institute for Chemistry and Biology of the Marine Environment, Carl von Ossietzky Universität \\ Oldenburg, Wilhelmshaven, Germany \\ ${ }^{6}$ Biology Sciences, The University of Auckland, New Zealand
}

Some sponge species are found both in shallow and deeper waters, however, the majority of sponges seems to prefer either shallow or deeper waters. It is currently unknown whether sponge-associated microorganisms play a role in the ability of their host to cover a larger depth range. Whereas the microbiota associated with shallow water sponges has been extensively studied during the past decade, microbial communities associated with sponges that chose habitats out of reach of SCUBA divers are still largely a 'blue hole'.

To address this knowledge gap, we selected four sponge species that are found both in shallow water $(0-30 \mathrm{~m})$ and in deeper waters $(60-90 \mathrm{~m})$. Two of these species were sampled in the Pacific (Callyspongia sp. and Rhabdastrella globostellata) and two in the Caribbean (Xestospongia muta and Agelas sventres). Next generation sequencing of PCR-amplified 16S rRNA gene fragments was applied to characterize microbial diversity in these sponges. In addition, antimicrobial activity is being determined from extracts and bacterial isolates obtained from Caribbean sponges sampled from different depths.

The first results for the Pacific sponge species and the seawater show depth-related stratification of the microbial communities. Perhaps not surprisingly, bacteria that were most important for the divide between shallow and deep individuals from the same species were cyanobacteria. However, the divide was not entirely explained by the disappearance of cyanobacteria in the deeper sponges. The highly reduced relative abundance of cyanobacterial species in the deeper sponges was accompanied by increased relative abundance of other cyanobacterial species suggesting that certain cyanobacteria are better adjusted to environmental conditions in the deeper waters. In addition, preliminary results of antimicrobial activity tests of organic extracts from the Caribbean sponges indicate that extracts from the shallow-water sponges are more active against Gram+ bacteria than extracts from their deeper counterparts. No depth-related differences in antimicrobial activity against Grambacteria were observed. 


\title{
INTESTINAL MICROBIOLOGY IN EARLY LIFE
}

\author{
ROCIO MARTIN ${ }^{1}$ (ROCIO.MARTIN@DANONE.COM)
}

${ }^{1}$ Danone Nutricia Research, Singapore

\begin{abstract}
The intestinal microbiota forms an integral part of normal human physiology, and in recent years disturbances of the normal gut microbiology have been linked to some important health and disease issues. Newborns are essentially sterile, but the complex, high density microbiota establishes from the very first minutes of life. The first colonizers play an important role in the development of the microbial ecosystem which may indeed steer the long-term composition and activity of the microbiota, and therefor also directly the symbiosis with the host that is so important for health. Considering the importance of the microbiota on the human immune, metabolic, and even neurological systems, it is important to understand the dynamics and driving determinants of this development.
\end{abstract}

Exclusive human milk feeding is considered the first choice of infant nutrition, not only providing optimal nutrition, but also bioactive components that are crucial for optimal gut development, immune maturation, metabolic development, and even cognitive development. Human milk also has an important impact on the microbiology of the gut as there are many components in human milk that have growth stimulating or growth inhibiting effects on the different types of micro-organisms that an infant can be exposed to. Besides the diet also host genetics, mode of delivery, the use of antibiotics and other variables can impact the early microbiota. Some of these microbiome drivers will be discussed, as will be the potential consequences for health in early and later life. 


\title{
EXPLORING ANAEROBIC MICROBIAL DEHALOGENATION REACTIONS APPLYING COMPOUND-SPECIFIC STABLE ISOTOPE ANALYSIS
}

\author{
IVONNE NIJENHUIS
}

Department of Isotope-Biogeochemistry, Helmholtz-Centre for Environmental Research UFZ, 04318 Leipzig, Germany

\begin{abstract}
Over the last decades, concepts involving compound-specific stable isotope analysis (CSIA) have been developed allowing the qualification and quantification of in situ (bio)transformation of common groundwater contaminants such as the chlorinated ethenes and ethanes. CSIA has additionally been shown to be a promising tool for the characterisation of the (bio)chemical reactions involved. Especially the recent advance in methods which now enables the analysis of carbon, chlorine, bromine and hydrogen isotope compositions of halogenated organic substances has provided valuable toolkit. During biotransformation, typical isotope fractionation patterns may be observed pointing to the involved reaction mechanism.

Over the recent years, we have applied dual- and triple-element stable isotope analysis to investigate microbial reductive dehalogenation reactions of chlorinated and brominated ethenes and ethanes. For example, triple-element stable isotope composition was applied to investigate the dihaloelimination reaction of 1,2-dichloroethane by two Dehalococcoides mccartyi strains. Furthermore, dual carbon-bromine stable isotope analysis was applied to distinguish transformation pathways of ethylene dibromine (1,2-dibromoethene). Comparison with abiotic reactions and/or complementary experiments with deuterated water allowed to provide a closer insight into these biochemical reactions. In this presentation, the overall concept will be presented and examples will be provided applying CSIA for evaluation of microbial dehalogenation reactions.
\end{abstract}




\title{
DEEP LOOK INTO DEEP PERMAFROST: MULTI-OMICS INSIGHTS TO CARBON CYCLING
}

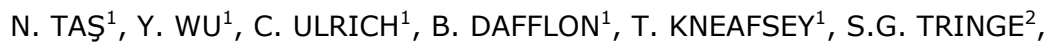 \\ S. HUBBARD ${ }^{1}$, J.K. JANSSON ${ }^{3}$ \\ ${ }^{1}$ Lawrence Berkeley National Laboratory, USA \\ ${ }^{2}$ DOE Joint Genome Institute (JGI), USA \\ ${ }^{3}$ Pacific Northwest National Laboratory, USA
}

\begin{abstract}
Permafrost soils are one of the world's largest terrestrial carbon storages thus an important focal point for climate change research. With increasing global temperatures, permafrost carbon stores may become available for rapid microbial mineralization and result in increased greenhouse gas (GHG) emissions. While carbon turnover at depth is proposed to be slower than surface, especially the fate of carbon in deep permafrost, which is currently protected from the warming climate, is uncertain. In this project, we applied metagenomics and metatranscriptomics to determine the phylogenetic and functional changes in the deep permafrost microbiome from polygonal arctic tundra at the Barrow Environmental Observatory (BEO) in Alaska. We collected permafrost cores ranging from $0.5-3 \mathrm{~m}$ in depth and analyzed over 200 samples along a transect containing high-, flat- and low-centered polygons in order to determine microbial responses to thaw. In this study, we present a series of labexperiments and field studies that combine the applications of next generation sequencing technologies with detailed measurements of soil structure and biogeochemistry to detect the response of deep soil microbes to environmental change in the Arctic. Besides depth, $\mathrm{pH}$ and changes in the material density were main drivers of microbial community structure in permafrost. Metagenomes from different permafrost depths also showed elevated potential for $\mathrm{CH}_{4}$ production in deeper layers while $\mathrm{CH}_{4}$ production and oxidation potential was detected in near surface permafrost. We hypothesize that nutrients and available carbon in these layers are tightly regulated and recycled where permafrost increasingly becomes a $\mathrm{CH}_{4}$ source with depth. Metagenomics coupled with reconstruction of microbial genomes, measurements of geochemistry and microbial processes aids us in understanding the biogeochemical cycles in Arctic soils and permafrost, and in the future will better inform efforts to resolve uncertainties surrounding ecosystem responses.
\end{abstract}




\title{
INTEGRATING (META)GENOMICS DATA INTO SPONGE SYMBIONT CULTIVATION
}

\author{
JOHANNA GUTLEBEN (JOHANNA.GUTLEBEN@WUR.NL), \\ CATARINA CAROLINA SALES E SANTOS LOUREIRO, HAUKE SMIDT, DETMER SIPKEMA
}

Laboratory of Microbiology, Stippeneng 4, 6708 WE Wageningen, The Netherlands

Introduction: Marine sponges (Porifera) harbour distinct and diverse microbial communities. These symbionts potentially produce sponge-derived, pharmaceutically interesting metabolites and hence cultivation of these microbes has gained focus in the scientific community. However, most microbial symbionts have evaded cultivation to date, thus a promising approach is to mine meta-omics data for in silico physiological traits that can be integrated in highthroughput cultivation strategies.

Goals: This study aimed to integrate genomic information into the design of novel cultivation media and develop a high-throughput experimental strategy targeting the cultivation and isolation of the sponge-associated candidate phylum Poribacteria. Additionally, this work set out to increase the cultivable fraction of the microbial community associated with the sponge Aplysina aerophoba.

Methodology: A collection of low-nutrient media was designed based on the predicted carbohydrate degradation metabolism of Poribacteria ${ }^{1,2}$. To further optimize the medium design, we aimed to mimic conditions from the sponge mesohyl niche by adding the spongederived antibiotic Aeroplysinin-1. In total, 240 cultivation conditions were created, and isolates were identified by $16 \mathrm{~S}$ rRNA gene amplicon sequencing.

Results: 1463 colony PCR products were analysed, resulting in a total of 128 cultivated OTUs. Amplicon sequencing revealed that no Poribacteria isolates were obtained. Nearly $70 \%$ of isolates were Alphaproteobacteria, of which Pseudovibrio and Ruegeria OTUs were the only two OTUs isolated from all media. Phylogenetic diversity varied significantly in different media and was influenced by medium dilution and the antibiotic Aeroplysinin-1, however, no novel OTUs were isolated in this study. Only $1.8 \%$ of the OTUs were observed in both the cultured fractions and the sponge community, which was dominated by Chloroflexi, Actinobacteria and Cyanobacteria.

Conclusion: The largest part of the sponge-associated microbial community remains uncultivated despite tailor made media designs and a high throughput cultivation approach. Nonetheless, the media formulations developed here have potential as enrichment media for specific microbial groups.

1. Siegl, A. et al. Single-cell genomics reveals the lifestyle of Poribacteria, a candidate phylum symbiotically associated with marine sponges. ISME J. 5, 61-70 (2011).

2. Kamke, J. et al. Single-cell genomics reveals complex carbohydrate degradation patterns in poribacterial symbionts of marine sponges. ISME J. 7, 2287-300 (2013). 


\section{Oral presentations Microbial physiology October 19, 2017}




\section{YEARS OF MICROBIOLOGY IN WAGENINGEN AND 50 YEARS OF SYNTROPHY: WHAT DID WE LEARN?}

BERNHARD SCHINK

University of Konstanz, Germany

The study of the complex microbial network catalyzing the conversion of biomass to methane and $\mathrm{CO}_{2}$ started in the last decades of the $19^{\text {th }}$ century and continues to provide exciting new insights into microbial interactions and their biochemical backgrounds. After isolation and characterization of pure cultures in the era of Robert Koch and his coworkers had been defined as an indispensable basis for reproducible scientific work on microbes the importance of interactions in natural and synthetic microbial communities gained interest only in the 1970s and 1980s when syntrophic interactions between phototrophic, fermenting and methanogenic microbes were discovered. Beyond the classical cases of syntrophic oxidation of ethanol, butyrate, propionate, and benzoate, also more complex substrates such as glucose or amino acids turned out to be degraded preferentially in syntrophic associations. While the biochemistry of substrate degradation in all these cases has been worked out to sufficient detail the bioenergetics of interspecies electron transfer through various carrier systems still provide sufficient problems for actual research. Hydrogen and formate have been considered to be equivalent electron carriers in syntrophic degradation processes in the past but may serve slightly different functions in these processes. At least for one example, evidence could be provided that formate conversion to hydrogen can be associated with a gain of metabolic energy, thus indicating that both carriers are not always energetically equivalent. The energetic situations of syntrophic microbial associations are different between biogas reactors and lake sediments. Especially in the latter case, the energy gains of the partners involved come down to ranges that ask for extensions of our present-day understanding of microbial energy metabolism. 


\title{
MICROBIAL PROCESSES IN THE DEEP SUBSURFACE
}

\author{
JAN DOLFING (JAN.DOLFING@NEWCASTLE.AC.UK)
}

Newcastle University, Newcastle upon Tyne School of Civil Engineering and Geosciences

\begin{abstract}
Being able to state on an American university campus that you are a "Dutch microbiologist" frequently elicits positive responses, to say the least. In this presentation, I will briefly outline how my background and training at the interface of environmental engineering and microbial ecology In Wageningen prepared me for "my best experiment ever", and link to one of my current projects on microbial electron transport at high pressure. The deep ocean is one of the largest microbial habitats on the planet. Hence, high hydrostatic pressure is a feature of microbial life. Changes in pressure can inhibit some reactions, whilst simultaneously accelerating others. Extracellular electron transfer (EET) is a process whereby microbes respire solid substrates in their surrounding environment. For an electron to move outside of the cell, it must transit the microbial envelope through a series of membrane bound electron carriers each of which will have a unique pressure response. We are developing an experimental system for studying EET under high pressure.
\end{abstract}




\title{
ANAEROBIC MICROBIAL GROWTH ON ONE-CARBON COMPOUNDS
}

\author{
MARTIJN DIENDER, JOÃO MOREIRA, ALFONS J.M. STAMS AND DIANA Z. SOUSA
}

Laboratory of Microbiology, Wageningen University, The Netherlands

\begin{abstract}
Syngas, a mixture of $\mathrm{CO}, \mathrm{H}_{2}$, and $\mathrm{CO}_{2}$, can be generated via gasification of virtually any carbonaceous material (including renewables, such as wastes and lignocellulosic biomass). Fermentation of syngas by carboxydotrophic (i.e. CO-utilizing) microbes allows for its conversion to added-value chemicals. Currently, the microbial production of ethanol from syngas is well established and commercialized, and there is interest to diversify production towards, for example, higher alcohols or organic acids. However, genetic engineering of carboxydotrophic organisms and the knowledge of their metabolism is still limited, making it difficult to develop strains producing these products.
\end{abstract}

In our research we attempt to construct microbial defined networks in which two (or more) microbes can produce a desired compound via a sequence of cross-feeding reactions. As a proof-of-concept for a syngas-converting microbial network, we performed co-cultivation of Clostridium autoethanogenum, a well-known carboxydotrophic acetogen, together with Clostridium kluyveri, a well characterized organism employing the reverse $\beta$-oxidation pathway. C. autoethanogenum uses the syngas to produce a mixture of acetate and ethanol, which are subsequently used by $C$. kluyveri to produce butyrate and caproate. Interestingly, C. autoethanogenum is again able to use these acids to convert them to their respective alcohols: butanol and hexanol. A second synthetic co-culture composed of an Acetobacterium $\mathrm{sp}$. and Pelobacter propionicus was constructed to convert $\mathrm{CO}$ to propionate. The objective is to produce odd-numbered fatty-acids by adding $C$. kluyveri to this co-culture.

The constructed synthetic co-cultures pose an interesting way of combining one-carbon metabolism with chain elongation, resulting in the production of more complex and valuable products from syngas. Physiological characterization of the co-cultures and interspecies interactions are currently being investigated. 


\title{
EXPLORATION OF SULFATE-REDUCING BACTERIA COLONIZING THE INTESTINAL TRACTS
}

\author{
W. IKEDA-OHTSUBO (WAKAKO.OHTSUBO@TOHOKU.AC.JP), \\ R. KUSAKA \\ Department of Agricultural Science, Tohoku University, Japan
}

Sulfate-reducing bacteria (SRB) occur in the intestinal tracts of various organisms, including human, animals, fish and insects. In human, several diseases and disorders, including ulcerative colitis (UC), colon cancer, metabolic syndrome, obesity, sudden infant death syndrome, and autism, have been reported to be associated with an increased number of SRB, while ecophysiology of intestinal SRB is largely unknown. In our present study, we investigated human intestinal mucosal specimens for the occurrence of SRB by culture-dependent and independent approach. While we failed to detect SRB in most samples by clone library analyses and PCR amplification of $d s r$ (gene encoding dissimilatory sulfite reductase), SRB was detected in culture enrichments using a medium containing acetate as an electron donor. The enriched strain was affiliated with an uncultured, deep-branching group, which is likely to represent a novel lineage of Desulfovibrionaceae. 


\title{
ISOLATION AND CHARACTERIZATION OF NOVEL ELECTROTROPHIC MICROORGANISMS USING METALLIC IRON AS SOLE ELECTRON DONOR
}

JO PHILIPS (JO.PHILIPS@UGENT.BE),

EVA MONBALLYU, STEFFEN GEORG, NIELS VAN DEN DRIESSCHE, KORNEEL RABAEY, JAN B.A. ARENDS

Center for Microbial Ecology and Technology (CMET), Ghent University, Coupure Links 653, 9000 Ghent, Belgium

\begin{abstract}
Some microorganisms are capable of using metallic iron as electron donor. This capacity leads to severe corrosion to metallic structures, but can also have interesting biotechnological applications. Microbial electrosynthesis (MES) depends on homoacetogens capable of deriving electrons from a cathode to reduce carbon dioxide to organic molecules. Both metallic iron and a cathode are solid state electron donors, therefore, the use of metallic iron as an electron donor for enrichments and isolations seems a promising strategy to obtain novel highperforming strains for microbial electrosynthesis. The goal of this work was to isolate acetogenic strains using metallic iron as electron donor from environmental rust samples. The rust layer of metallic waste present in a local river was scraped off and used to set up enrichments. Metallic iron particles were added as sole electron donor and methanogenic growth was inhibited. Isolation was performed using agar plates containing iron powder, while agar plates with an organic substrate were used for further purification. Most isolates were strongly related (99\%) to Acetobacterium malicum, while also a Sporomusa and Shewanella strain were obtained. The new strains enhance $\mathrm{Fe}(0)$ corrosion at least five times in comparison to abiotic corrosion. Consequently, these strains must have a mechanism for direct electron uptake or the ability to enhance abiotic hydrogen evolution. Insights into the extracellular electron uptake mechanisms, as well as their capacity for microbial electrosynthesis will be presented. The study of these $\mathrm{Fe}(0)$ oxidizing strains will lead to new insights into biocorrosion and extracellular electron uptake mechanisms and will contribute to the improvement of biofilm based microbial electrosynthesis.
\end{abstract}




\title{
GALACTURONATE FERMENTATION BY ANAEROBIC CHEMOSTAT ENRICHMENT CULTURES
}

\author{
LAURA C. VALK (L.C.VALK@TUDELFT.NL), \\ MARK C.M. VAN LOOSDRECHT AND JACK T. PRONK \\ Department of Biotechnology, Delft University of Technology, Delft, The Netherlands
}

\begin{abstract}
Conversion of plant-derived waste streams from agriculture and food processing by open, mixed microbial populations is gaining increasing interest. This approach is not only applicable for wastewater treatment but also for producing a variety of platform chemicals. Sugar-beet pulp and citrus peel are agricultural waste streams that are rich in pectin, a polymer that contains galacturonic acid as a main constituent. These large pectin-rich streams represent a potential, currently underexplored, feedstock for production of bulk chemicals or biofuels. The anaerobic, fermentative, conversion of this carboxylated sugar by enrichment cultures has not yet been studied in bioreactors in chemostat operations. The aim of this study was to characterize product profiles and population composition of open, mixed-culture anaerobic chemostat cultures fed with D-galacturonate as sole limiting substrate. 16s-rRNA data showed that stable enrichments were obtained, with similar microbial communities in biological replicate experiments. The populations were dominated by an uncultured Lachnotalea bacterium, with smaller populations of Klebsiella oxytoca and an uncultured Enterobacter bacterium. Acetate was the major catabolic product of the mixed cultures, with additional formation of formate and hydrogen. However, the expected 1:1 molar ratio of acetate to hydrogen and formate was not observed. This result could be explained by the occurrence of acetogenesis via the Wood-Ljungdahl pathway. Indeed, metagenome analysis revealed the presence of key genes of this pathway. Enrichment of acetogenic bacteria was not observed in previous studies in which similar anaerobic enrichments were performed with glucose as the substrate. This difference was attributed to a larger relative impact of the Wood-Ljungdahl pathway on Gibbs-free-energy and chemiosmotic energy conservation during growth on D-galacturonate.
\end{abstract}

This research was funded by the SIAM Gravitation Grant 024.002.002, the Netherlands Organization for Scientific Research. 


\title{
HARNESSING MICROBIAL EVOLVABILITY TOWARDS DEVELOPING STABLE PHOTOSYNTHETIC CELL FACTORIES
}

\author{
WEI DU (W.DU1@UVA.NL), \\ JOERI A. JONGBLOETS, KLAAS J. HELLINGWERF, \\ FILIPE BRANCO DOS SANTOS
}

Molecular Microbial Physiology Group, Swammerdam Institute for Life Sciences, University of Amsterdam, Science Park 904, Amsterdam 1098 XH, The Netherlands

\begin{abstract}
Microbial bioengineering could potentially contribute to the future development of human society by providing sustainable, novel, and cost-effective production pipelines. However, the sustained productivity of genetically engineered bacteria is often a challenge. This is because traditional genetic engineering strategies for microbial product formation, i.e. through introduction of heterologous pathways consisting of multiple genes with modulated expression levels, often are burdensome for cell growth. Consequently, spontaneous non-producing mutants tend to grow faster, gradually taking over the population and undermining the total productivity of the culture. To prevent this issue of strain instability, we propose a novel strategy applicable to all microbial production systems for which a genome-scale metabolic model (GSM) is available, that couples native metabolite production with biomass formation. We developed an algorithm that 'Finds Reactions Usable In Tapping Side-products' (FRUITS), which identifies compounds that are suitable to be coupled to cell growth by blocking their respective re-utilization pathway(s). When applied to Synechocystis sp. PCC6803, a model cyanobacterium extensively explored for sustainable bioproduction, a total of nine target metabolites were identified. We tested our approach for one of these compounds, acetate, which is used in a wide-range of industrial applications and is easy to quantify. The modelguided engineered strain shows an obligatory coupling between acetate production and photoautotrophic growth as predicted. Furthermore, the stability of acetate productivity in this strain was confirmed by performing prolonged turbidostat cultivations. This work demonstrates a novel approach to stabilize the production of target compounds in cyanobacteria that culminated in the first report of a photoautotrophic growth-coupled cell factory. The method developed is generic and can easily be extended to any other microbial production system for which a GSM is available.
\end{abstract}




\title{
PHARMACEUTICAL BIODEGRADATION UNDER THREE ANAEROBIC REDOX CONDITIONS EVALUATED BY CHEMICAL AND TOXICOLOGICAL ANALYSES
}

\author{
YUJIE HE, NORA SUTTON, HUUB RIJNAARTS, \\ ALETTE LANGENHOFF (ALETTE.LANGENHOFF@WUR.NL) \\ Department of Environmental Technology, Wageningen University and Research, \\ P.O. Box 17, 6700 AA Wageningen, The Netherlands
}

Biodegradation of pharmaceutically active compounds (PhACs) in the subsurface layer of constructed wetlands (CWs) under various anaerobic redox conditions is rarely studied. In this study, CW sediment microbial populations were enriched for PhAC biodegrading organisms. Biodegradation effectivity of a mixture of six PhACs (caffeine, CAF; naproxen, NAP; metoprolol, MET; propranolol, PRO; ibuprofen, IBP; carbamazepine, CBZ) and single compounds (CAF, NAP) was investigated under nitrate reducing, sulphate reducing, and methanogenic conditions using chemical and toxicological analyses.

Biodegradation efficiencies varied strongly among the six PhACs and three redox conditions chosen. CAF and NAP were completely biodegraded under sulfate reducing and methanogenic conditions whereas biodegradation efficiencies of the other PhACs were much less (MET, PRO $<20 \%$; IBP, CBZ, negligible). CAF and NAP showed significantly lower biodegradation under nitrate reducing conditions than under the other two redox conditions. No difference was found in biodegradation efficiencies of CAF and NAP when present as single compound, or as a mixture with other PhACs.

Different intermediates were observed, indicating different biodegradation pathways under different redox conditions and when the PhACs were present as single compound or in a mixture. From toxicological perspective, toxicity of PhACs and/or their intermediates to Vibrio fischeri was attenuated during the biodegradation process.

Chemical and toxicological data showed positive correlations in principle component analysis, by which potentially toxic PhACs and intermediates are indicated for further ecotoxicological hazard assessment.

This research is a first step towards increasing our understanding of PhAC biodegradation processes in the subsurface layers of CWs, and their contribution to reduce hazardous effects of PhACs and intermediates to aquatic ecosystems. 


\section{Oral presentations Bacterial genetics October 20, 2017}




\title{
THE ORIGIN OF COMPLEX CELLS
}

THIJS ETTEMA (THIJS.ETTEMA@ICM.UU.SE)

Uppsala University, Sweden

\begin{abstract}
The origin and early evolution of eukaryotes represents one of the most contentious puzzles in modern biology. The past decade has seen a shift in the traditional view of how eukaryotic cells evolved. Whereas previously they were thought have evolved as a separate lineage, in accordance with Carl Woese's three domains Tree of Life, recent analyses indicate that the eukaryotic lineage evolved from within the archaeal domain. Moreover, the discovery of novel archaeal lineages indicate that this archaeal ancestor might have been more complex than was presumed before. The recent discovery of the Lokiarchaeota, an archaeal lineage that forms a clade with eukaryotes and whose genome encodes several unexpected eukaryotic features, has underscored this notion. These findings have opened up new avenues for discussing eukaryotic origins: How 'complex' was the archaeal ancestor of eukaryotes? When in the process of eukaryogenesis did mitochondria evolve? How did eukaryotic gene content gain shape, and which evolutionary forces were driving this process? Here, I will focus on keyplayers in the process of eukaryogenesis: The archaeal 'host cell' and the mitochondrial endosymbiont. Recent data produced by my lab has allowed us to trace the identity and nature of these lineages, allowing us to reconstruct the events that led to the origin of complex cell types.
\end{abstract}




\title{
A BRIEF HISTORY OF CRISPR RESEARCH
}

\author{
STAN J.J. BROUNS
}

Department of Bionanoscience, Kavli institute of Nanoscience, Delft University of Technology, The Netherlands

Laboratory of Microbiology, Wageningen University, The Netherlands

The CRISPR immune system protects bacteria and archaea from invading viruses and plasmids. Immunity depends on protein complexes that use small RNA molecules to find matching viral or plasmid DNA. I will give a brief overview of the CRISPR research carried out in Wageningen, and will go through some of the breakthrough discoveries including the discovery of the Cascade complex. I will show how this complex produces small guide RNAs that drive the immune system, and give some structure insights into this unique Cas protein machinery. I will present a mechanism called priming that takes care of mutated escaped viruses and will quickly update the memory of the immune system. All of these insights into the biology of CRISPR have led to some of the most revolutionary molecular genetics tools to date, with Cas9 being the most well known example. I will highlight some new CRISPR tools for genome engineering approaches to edit genomes, knockdown gene expression, and allow for sensitive diagnostics applications. 


\title{
BACILLUS SMITHII AS A NOVEL THERMOPHILIC CELL FACTORY: FROM ISOLATION TO ENGINEERING
}

\author{
ELLEKE F. BOSMA
}

Denmark Technical University, Copenhagen, DK

This presentation will give an overview of our work on the isolation, selection and metabolic engineering of a thermophilic bacterium for green chemical production, with a focus on genetic tool development for non-model and thermophilic microorganisms. Whereas thermophilic bacteria are attractive production hosts for cost-efficient and sustainable production of green chemicals from renewable resources, their development into versatile cell factories is hindered by their poor genetic accessibility and lack of efficient genetic tools. Therefore, in this project we started by isolating a collection of thermophilic bacilli from a compost heap and screened for genetic accessibility, as well as other industrially relevant traits such as substrate utilization and fermentation capacities. The most promising organism selected for all further work was Bacillus smithii ET 138 , a facultative anaerobe growing between $37^{\circ} \mathrm{C}$ and $65^{\circ} \mathrm{C}$ with an optimum at $55^{\circ} \mathrm{C}$. After genome sequencing and metabolic reconstruction, we developed basic genetic tools for gene deletion and overexpression, constructed several mutant strains and gained further insight into the strain's metabolism via transcriptomics and metabolomics. Our recent efforts have focused on acceleration of the engineering process by creating efficient CRISPR-Cas9-based genome editing and silencing tools. Our discovery that the widely used S. pyogenes Cas9 (SpCas9) is inactive in vivo $\geq 42^{\circ} \mathrm{C}$ allowed for temperature-based control of SpCas9-activity by using the wide growth temperature range of $B$. smithii. This resulted in a highly efficient genome editing tool using SpCas9 as a counter-selection after plasmid-based homologous recombination. Next, we identified and characterized ThermoCas9: a thermostable Cas9-orthologue from Geobacillus thermodenitrificans that is active at a wide temperature range and with which we established the first Cas9-based genome editing and silencing tool at thermophilic temperatures. Both our Cas9-based tools will open new doors for studying, engineering and using non-model and thermophilic production hosts. We are currently using these tools to gain better understanding of the metabolism of $B$. smithii ET 138 and engineer it towards desired products. 


\title{
CONVERTING ESCHERICHIA COLI INTO AN "ARCHAEBACTERIUM" WITH A HYBRID HETEROCHIRAL MEMBRANE
}

MELVIN SILIAKUS

Netherlands Institute for Sea Research, Texel, The Netherlands

\begin{abstract}
A frequently-quoted hypothesis on the origin of the three domains of life assumes the existence of a last universal common ancestor (LUCA) from which bacteria and archaea have diverged. Membrane lipids of bacteria and archaea are based on glycerol-3-phosphate esters and glycerol-1-phosphate ethers, respectively. This striking difference has led to the divergent hypothesis of a non-cellular LUCA, that either lacked a membrane, or possessed a mineral membrane. An alternative model assumes that LUCA contained a defined cellular membrane. Membrane synthesis in proto-cells was probably catalyzed both by abiotic catalysis and by enzymes with poor stereoselectivity, and hence membranes might have consisted of mixtures of G1P and G3P lipids. However, proto-cells with such heterochiral membranes have been proposed to be relatively unstable and would evolve into more stable homochiral membranes through evolution of stereospecific enzymes. Although some attempts have been made to reproduce an in vivo heterochiral membrane by introducing archaeal lipid biosynthesis enzymes into Escherichia coli, this has resulted in only minor levels of ether lipids. Here we report the engineering of $E$. coli with a hybrid heterochiral membrane containing substantial amounts of archaeal lipids ( $>20 \%$ ), yielding viable bacterial cells for multiple generations. Moreover, we demonstrate substrate promiscuity of key archaeal lipid biosynthesis enzymes. These results reveal that hybrid heterochiral membranes are stable and compatible with cellular life, implying that this state may have existed at some stage in the evolution.
\end{abstract}




\title{
MONASCUS RUBER AS CELL FACTORY FOR LACTIC ACID PRODUCTION AT LOW PH
}

\author{
RUUD A. WEUSTHUIS
}

BioProcess Engineering, Wageningen University \& Research, The Netherlands

\begin{abstract}
A Monascus ruber strain was isolated that was able to grow on mineral medium at high sugar concentrations and $175 \mathrm{~g} / \mathrm{I}$ lactic acid at $\mathrm{pH} 2.8$. Its genome and transcriptomes were sequenced and annotated. Genes encoding lactate dehydrogenase (LDH) were introduced to accomplish lactic acid production and two genes encoding pyruvate decarboxylase (PDC) were knocked out to subdue ethanol formation. The strain preferred lactic acid to glucose as carbon source, which hampered glucose consumption and therefore also lactic acid production. Lactic acid consumption was stopped by knocking out 4 cytochrome-dependent LDH (CLDH) genes, and evolutionary engineering was used to increase the glucose consumption rate. Application of this strain in a fed-batch fermentation resulted in a maximum lactic acid titer of $190 \mathrm{~g} / \mathrm{l}$ at $\mathrm{pH} 3.8$ and $129 \mathrm{~g} / \mathrm{l}$ at $\mathrm{pH} 2.8$, respectively 1.7 and 2.2 times higher than reported in literature before. Yield and productivity were on par with the best strains described in literature for lactic acid production at low $\mathrm{pH}$.
\end{abstract}




\title{
PROGRAMMABLE CRISPR-CAS9 ROADBLOCKS TO STUDY MECHANISMS IN DNA MISMATCH REPAIR
}

JOYCE H.G. LEBBINK

Erasmus MC, Rotterdam, The Netherlands

\begin{abstract}
Many DNA transactions responsible for maintaining genome stability, require communication between distant sites on DNA. Studies unraveling the mechanism of these communication processes often employ protein roadblocks to distinguish between communication along the protein backbone and coupling of sites through three-dimensional space (for example loop formation). Precise placement of these roadblocks onto DNA repair substrates requires the introduction of specific recognition sites into the DNA. Here we bypass this requirement by using RNA-guided nuclease-dead Cas9 as in vitro roadblock. By varying crRNA sequences, we were able to put dCas9 blocks on different PAM-containing locations on DNA substrates used in in vitro reconstituted DNA mismatch repair (MMR) reactions. In these reactions, MMR proteins couple detection of a mismatch to localization and incision of a pre-existing strand discrimination signal further away along the DNA We find that programming dCas9 to target different locations on the mismatch-containing DNA results in radically different incision patterns. These results will provide the urgently required functional validation for hypothesized diffusion-based communication along the DNA backbone during DNA mismatch repair. These preliminary findings showcase the potential of Cas9 and its variants as molecular in vitro tools to unravel molecular mechanisms in genome maintenance pathways relevant for human health.
\end{abstract}




\title{
100 YEARS OF "IMPOSSIBLE" ANAEROBIC MICROBIOLOGY AND BEYOND
}

\author{
MIKE JETTEN (M.JETTEN@SCIENCE.RU.NL)
}

Soehngen Institute of Anaerobic Microbiology, and Radboud University, Nijmegen, The Netherlands

The world is a microbial planet, where microbes fulfil many beneficial roles, and only very few are pathogens. Microbial life arose about 4 Gy ago with anaerobic methanogens and acetogens as one of the first metabolisms. For almost 2 Gy life was almost exclusively anaerobic, and to date still many anaerobic micro-organisms are important for wastewater treatment, in producing fermentation products and sustaining human health. Honouring Nicolas Soehngen, the first Dutch microbiology PhD student, and later first Professor of Microbiology at Wageningen University, we have established the Soehngen Institute of Anaerobic Microbiology (SIAM). At SIAM we want to continue the excellent tradition of Microbiology in the Netherlands and aim to discover and apply new "impossible" anaerobic microorganisms. Several examples of new discoveries in the nitrogen and methane cycles will be highlighted. Anaerobic oxidation of ammonium by anammox bacteria was once deemed to be biochemically impossible and nonexistent in nature, but has now been identified as important player in global nitrogen cycling. Molecular studies show that anammox bacteria can make the rocket fuel hydrazine by novel protein complexes that are located in a unique bacterial organelle surrounded by ladderane lipids. Anammox is applied both in side stream and main stream waste treatment, circumventing the use of oxygen and organic carbon and preventing the emission of greenhouse gasses. Another example is the recently discovered comammox process carried out by new Nitrospira bacteria that oxidize ammonium into nitrate. Following in Soehngens -on the origin and disappearance of methane- footsteps, SIAM investigates new methanogens and methanotrophs. Special attention is given to anaerobic oxidation of methane (AOM) by archaea and bacteria. Nitrite-AOM is mediated by so called Methylomirabilis. oxyfera bacteria that turned out to have a new intra-aerobic metabolism. They are able to produce their own oxygen by conversion of $2 \mathrm{NO}$ into $\mathrm{O} 2$ and $\mathrm{N} 2$ by a putative NO dismutase. New Methanoperedens archaea catalyse nitrate-AOM and iron-AOM via reverse methanogenesis. Also AOM can be applied in waste treatment in combination with anammox or oxygen limited nitrification. The carbon that is not used in the processes can be converted into biogas or into bacterial storage material that can be used in the production of biodegradable plastics. Molecular surveys have indicated that many of these organisms are wide spread in anaerobic ecosystems around the globe where they most probably interact in an intricate anaerobic food chain. For more information see www.anaerobic-microbiology.eu 


\section{Poster pitches \\ October 19, 2017 \\ 12.00-12.10 hrs}




\title{
A BENZENE-DEGRADING NITRATE-REDUCING MICROBIAL CONSORTIUM DISPLAYS AEROBIC AND ANAEROBIC BENZENE DGERADATION PATHWAYS
}

\author{
S. ATASHGAHI ${ }^{1}$ (SIAVASH.ATASHGAHI@WUR.NL), \\ B. HORNUNG ${ }^{1}$, M.J. VAN DER WAALS ${ }^{1,2}$, R. VAN SPANNING ${ }^{3}$, A.J.M. STAMS ${ }^{1}$, \\ J. GERRITSE ${ }^{2}, \mathrm{H}$. SMIDT $^{1}$ \\ ${ }^{1}$ Wageningen University \& Research, Laboratory of Microbiology, Stippeneng 4, \\ 6708 WE Wageningen, The Netherlands \\ 2 Deltares, Subsurface and Groundwater Systems, Princetonlaan 6, 3584 CB Utrecht, \\ The Netherlands \\ ${ }^{3}$ Vrije Universiteit Amsterdam, Department of Molecular Cell Biology, De Boelelaan 1108, \\ $1081 \mathrm{HZ}$ Amsterdam, The Netherlands
}

\begin{abstract}
Microbial benzene oxidation proceeds either through aerobic or anaerobic pathways. In this study, we report transcription of aerobic and anaerobic benzene degradation pathways in a benzene-degrading denitrifying continuous culture. Transcripts associated with the family Peptococcaceae dominated all samples (21-36\% relative abundance) suggestive of their key role in the community. We found a highly-transcribed gene cluster encoding a presumed anaerobic benzene carboxylase ( $A b c A$ and $A b c D$ ) and a benzoate-coenzyme $A$ ligase (BzIA). Predicted gene products showed $>96 \%$ amino acid identity and similar gene order to the corresponding benzene degradation gene cluster described previously, providing further evidence for anaerobic benzene activation via carboxylation. For subsequent benzoyl-CoA dearomatization, bam-like genes analogous to the ones found in other strict anaerobes were transcribed, whereas gene transcripts involved in downstream benzoyl-CoA degradation were mostly analogous to the ones described in facultative anaerobes. The concurrent transcription of genes for enzymes involved in oxygenase-mediated aerobic benzene degradation suggested oxygen presence in the culture, possibly formed via a recently identified nitric oxide dismutase. Although we were unable to detect transcription of genes encoding such a nitric oxide dismutase, oxygen was produced upon addition of nitrite and formate to the continuous culture. Such an intermediate oxygen production would enable aerobic microbes to thrive in oxygen-depleted subsurface environments contaminated with hydrocarbons.
\end{abstract}




\title{
METATRANSCRIPTOMICS STUDY OF TROPHIC INTERACTION BETWEEN AKKERMANSIA MUCINIPHILA AND BUTYROGENIC GUT COMMENSALS
}

\author{
LOO WEE CHIA ${ }^{1}$ (LOOWEE.CHIA@WUR.NL), \\ BASTIAN V.H.K. HORNUNG ${ }^{1,2}$, STEVEN AALVINK ${ }^{1}$, PETER J. SCHAAP ${ }^{2}$, \\ WILLEM M. DE VOS ${ }^{1,3}$, JAN KNOL ${ }^{1,4}$, CLARA BELZER ${ }^{1}$ \\ ${ }^{1}$ Laboratory of Microbiology, Wageningen University \& Research, Wageningen, \\ The Netherlands \\ ${ }^{2}$ Laboratory of Systems and Synthetic Biology, Wageningen University \& Research, \\ Wageningen, The Netherlands \\ ${ }^{3}$ RPU Immunobiology, Faculty of Medicine, University of Helsinki, Helsinki, Finland \\ ${ }^{4}$ Nutricia Research, Utrecht, The Netherlands
}

Host glycans are paramount in regulating the symbiotic relationship between humans and their gut bacteria. The constant flux of host-secreted mucin at the mucosal layer creates a steady niche space for bacterial colonization. Mucin degradation by keystone species subsequently drives the local trophic chain and shapes mucosal microbial assembly.

This study investigated the in vitro molecular response of mucin-driven trophic interaction between the specialized mucin-degrader, Akkermansia muciniphila and butyrogenic gut commensals. A.muciniphila monoculture and co-cultures with non-mucolytic butyrogens from the family of Lachnospiraceae (Anaerostipes caccae and Eubacterium hallii) were grown in minimal media supplemented with pure mucin followed by determination of growth, metabolic end products (HPLC analysis) and meta-transcriptome (RNA-seq analysis).

We found that mucin degradation by A.muciniphila supported the growth of butyrogens and resulted in butyrate and propionate production. We identified, based on gene expression analysis, which pathways gave rise to the observed metabolic profiles. Butyrate was produced by butyrogens predominantly via acetyl-CoA pathway, which accounted for over $2 \%$ of the total transcripts. Propionate was produced by A.muciniphila via methylmalonyl-CoA pathway from succinate with the aid of vitamin B12 produced by E.hallii. In addition, E.hallii also produced propionate from 1,2-propanediol released by $A$. muciniphila via fucose degradation. Differential analysis (DESeq2) showed both butyrogens induced similar global transcriptional changes in A.muciniphila, with increased expression of mucin degradation genes and reduced expression of ribosomal genes. Interestingly, two putative operons and several two-component systems were differentially regulated, indicated the mode of transcriptional regulation by A.muciniphila in response to community members. E.hallii in particular, incurred a higher fold change on the differential expression of A.muciniphila than A.caccae.

This study deciphered the underlying mechanism of mucin-driven microbial tropism and demonstrated beneficial propionate production by the trophic interaction between A.muciniphila and E.hallii. Fucose metabolism by the mucosal symbionts could provide colonization resistance against fucose-dependent pathogens. 


\title{
CYCLIC COEVOLUTIONARY INTERACTIONS, COEXISTENCE FIXED POINT AND BIODIVERSITY CONTROL: AN EXAMPLARIC MINIMAL MODEL OF MUTUALISMS AND FEEDBACK
}

\author{
J.C. CLAUSSEN (J.CLAUSSEN@JACOBS-UNIVERSITY.DE) \\ Computational Systems Biology, Jacobs University Bremen
}

\begin{abstract}
Cyclic coevolutionary dynamics arises on many levels from bacteria in colicin biology to lizards and humans. Here I consider specifically evolutionary dynamics in the frameworks of LotkaVolterra systems and replicator equations in evolutionary game theory. These can include fixed points and cycles which can be neutrally stable, repelling or attracting. Of particular interest is the case where a coexistence state loses stability due to non-zero-sum payoffs, corresponding to dissipative interactions. Here I introduce a general ansatz of feedback control where an additive control term is implemented in the payoff matrix which is chosen proportional to an observable of the system which is suitably chosen to reflect the distance from the fixed point. This feedback control is implemented for the cyclic interaction model of Rock-Paper-Scissors type, for which the loss of stability depending on payoffs and population size has been recently discussed [J. C. Claussen and A. Traulsen, Physical Review Letters 100, 058104 (2008)]. As the discretization stochasticity in a finite population additionally destabilizes coexistence, a feedback control scheme can be introduced [J. C. Claussen, Evolutionary Dynamics: How Payoffs and Global Feedback Control the Stability, pp. 461-470 in: Control of Self-Organizing Nonlinear Systems, Springer (2016)]. Specifically, this implementation by a pairwise comparison process demonstrates that the control scheme is applicable even in a finite population. This minimal model can be considered as a first step towards control of desired states of higher biodiversity, but limitations arise from the type of feedback into system parameters, as growth rates or metabolic costs.
\end{abstract}


ISOLATION OF BACTERIOPHAGES AGAINST AKKERMANSIA MUCINIPHILA

\author{
S. GEERLINGS (SHARON.GEERLINGS@WUR.NL), \\ K. VAN DER ARK, Y. ZHU, W.M. DE VOS, C. BELZER
}

Laboratory of Microbiology, Wageningen University and Research, Wageningen, The Netherlands

\begin{abstract}
The human intestinal tract harbours up to 100 trillion microorganisms including Akkermansia muciniphila. A. muciniphila is a member of the phylum Verrucomicrobia and was found to be present in the intestinal tract of infants, adults and elderly. This species makes up $1-4 \%$ of the bacteria in the gut and its presence has been correlated to health in several studies. However, the genome of $A$. muciniphila is not genetically accessible yet using conventional transformation techniques. A different approach to alter a bacterial genome is transduction, using a bacteriophage (viruses able to infect bacteria). Therefore, the aim of this research is to isolate a bacteriophage able to infect $A$. muciniphila.
\end{abstract}

Three $A$. muciniphila strains ( $\mathrm{H} 1, \mathrm{H} 6$ and type strain $\mathrm{Muc}^{\top}$ ) were used to screen faeces samples on the presence of bacteriophages. Bacteriophages isolations from faeces samples were performed using two different methods: filtration and an enrichment method followed by chloroform treatment. Screening was executed using plaque assay and liquid culture propagations followed by transmission electron microscopy (TEM). Lastly, DNA was isolated from liquid cultures containing phage-like particles and sequenced.

Growth inhibition of $A$. muciniphila was observed in plaque assays with several bacteriophage isolates originating from different faeces samples. One of these bacteriophage isolates showed to inhibit $A$. muciniphila $\mathrm{H} 1$ and $\mathrm{Muc}^{\top}$ repeatedly in plaque assays and resulted in an altered morphology in liquid cultures. In addition, phage like particles were observed using TEM. DNA extraction and de novo sequencing did not result in the genome sequence of a bacteriophage.

The presence of a bacteriophage able to infect $A$. muciniphila could not be demonstrated yet. However, optimization of the viral DNA extraction protocol could lead to successful sequencing of a bacteriophage genome. 


\title{
MUCOLYTIC ACTIVITY OF AKKERMANSIA MUCINIPHILA ENABLES HUMAN MILK OLIGOSACCHARIDES DEGRADATION
}

\author{
IOANNIS KOSTOPOULOS ${ }^{1 *}$ (IOANNIS.KOSTOPOULOS@WUR.NL), \\ NOORA OTTMAN ${ }^{1 *}$, MARK DAVIDS $^{1}$, JAN KNOL $^{1,3}$, WILLEM M. DE VOS ${ }^{1,2}$ AND CLARA BELZER ${ }^{1}$ \\ ${ }^{1}$ Laboratory of Microbiology, Wageningen University, Stippeneng 4, 6708 WE Wageningen, \\ The Netherlands \\ ${ }^{2}$ Department of Basic Veterinary Medicine, Division of Microbiology and Epidemiology, \\ University of Helsinki, P.O.Box 66, FI-00014, Helsinki, Finland \\ ${ }^{3}$ Danone Nutricia Research, Uppsalalaan 12, 3584 CT Utrecht, The Netherlands \\ * These authors contributed equally to this study.
}

Introduction and objectives: Akkermansia muciniphila is a member of the intestinal microbiota specializes in mucus degradation. It is found to be abundant in healthy mucosa while it is inversely correlated to intestinal disorders such as IBD, Crohn's disease, ulcerative colitis and appendicitis, rendering $A$. muciniphila a potential biomarker for a healthy intestine. The enzymes that $A$. muciniphila harbours to degrade mucin can target similar structures in Human Milk Oligosaccharides (HMOs). This could explain the presence of this organism in the early life intestine. The aim of this study is to evaluate the ability of $A$. muciniphila to degrade HMOs.

Materials and Methods: $A$. muciniphila was grown on basal medium supplemented with human milk or HMOs as sole carbon and nitrogen source. Microbial growth was determined by plating $(\mathrm{CFU} / \mathrm{ml})$ and optical density $(600 \mathrm{~nm})$ while the fermentation products were analysed by HPLC. Moreover, selected samples were processed for transcriptomic analysis by RNA-Seq.

Results and Discussion: After 8 hours of incubation with human milk, growth of A. muciniphila increase of 5 -fold in CFU counts. HPLC analysis revealed that propionate and acetate were produced. On top of this, monomeric sugars were released, transcriptome analysis by RNA-Seq showed upregulation of genes involved in lactose degradation, carbohydrate transport and translational activity in A. muciniphila grown on human milk in comparison to mucin. However, the expression of genes involved in mucin degradation was not affected by the experimental conditions, suggesting that $A$. muciniphila is capable of using these genes also for utilization of human milk glycans. The capacity to survive in the early life environment by degrading and consuming human milk components might be beneficial for A. muciniphila during initial colonization before reaching the mucosal layer in the intestine. 


\title{
THE LIVING ENVIRONMENT SHAPES INTESTINAL MICROBIAL COMMUNITIES AND IMMUNE RESPONSES IN A MOUSE MODEL
}

\author{
N. OTTMAN ${ }^{1,2 *}$ (NOORA.OTTMAN@KI.SE), \\ L. RUOKOLAINEN ${ }^{2}$, A. SUOMALAINEN ${ }^{3}$, P. KARISOLA ${ }^{3}$, J. LEHTIMÄKI ${ }^{2}$,

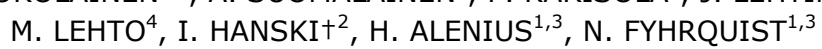 \\ *equal contribution \\ t deceased during the preparation of this manuscript in May 2016 \\ ${ }^{1}$ Institute of Environmental Medicine, Karolinska Institutet, Sweden \\ 2 Department of Biosciences, University of Helsinki, Finland \\ ${ }^{3}$ Department of Bacteriology and Immunology, University of Helsinki, Finland \\ ${ }^{4}$ Finnish Institute of Occupational Health, Finland
}

Contact with environmental biodiversity has been suggested to be protective against allergies. In particular exposure to soil, and the rich microbiota within, is proposed to be important for health. The research performed so far provides only some clues to understanding the connection between immune tolerance and microbial colonization from the environment. Here, we aim at gaining more direct evidence of the "environment-microbiota-health axis" by studying the colonization of gut microbiota in mice, after exposure to soil, and examining the immune status in both a steady state situation and during inflammation.

The small intestinal and fecal microbiota of mice kept on a clean bedding or in contact with soil was analysed, and the data were combined with immune parameters of the mice. Both healthy mice and those exposed to the murine asthma model were used in the study. We observe marked differences in the gut microbial composition between the living environments, with a higher proportion of Bacteroidetes in the soil group. The living environment also influenced mouse intestinal gene expression as shown by up-regulated expression of IL-10, Foxp3 and CD86 in the soil group. Furthermore, using the murine acute lung inflammation model we found that exposure to soil polarizes the immune system towards Th1 and a higher level of anti-inflammatory signaling, alleviating Th2 type allergic responses. Interestingly, the inflammatory status of the mice strongly influenced the composition of the fecal microbiota, overriding for the most part the effect of environmental exposures.

In conclusion, the soil environment clearly affects the composition of murine gut microbiota and immune status. Our results provide direct evidence of the role of environmentally acquired microbes in protecting against inflammation. Moreover, our study provides additional evidence of the bidirectionality of the gut-lung axis, and the ability of these organs to influence each other's immune responses and microbiota composition. 


\title{
MICROBIAL DIVERSITY IN XYLOSE OR GLUCOSE FERMENTING ENRICHMENTS: THE IMPACT OF CONTINUOUS GROWTH LIMITATION
}

\author{
J.L. ROMBOUTS (J.L.ROMBOUTS@TUDELFT.NL), \\ G. MOS, D.G. WEISSBRODT, R. KLEEREBEZEM, M.C.M. VAN LOOSDRECHT
}

Department of Biotechnology, Delft University of Technology, The Netherlands

\begin{abstract}
Fermentation is a common trait among bacteria. A fraction of $10 \%$ of reference populations in the KEGG database harbor one or more essential fermentative genes in their genome. 16S rRNA gene based sequencing techniques provide fast high throughput analysis and detailed insight in microbial ecosystems, such as carbohydrates-fermenting communities. Microbial diversity in this fermentative niche is usually high, even in simple ecosystems with only monosaccharides as carbon substrate. This conflicts with the classic idea of niche exclusion, where a microorganism with the highest competitive advantage will eventually dominate. In sugar fermentation, such advantage either relates to highest maximum biomass specific rate of growth $\left(\mu^{\text {max }}\right)$ in pulse-fed systems such as a sequencing batch reactor (SBR). Or to highest affinity in continuous-flow stirred tank reactors (CSTRs), where the substrate supply is continuously growth rate limiting.
\end{abstract}

Here, lab-scale mesophilic $\left(T=30^{\circ} \mathrm{C}\right)$ bioreactor experiments were conducted at slightly alkaline $\mathrm{pH}(8.0)$ and short solid retention time (SRT $=8 \mathrm{~h}$ ) to establish a selective niche for fermentation of xylose or glucose. Combination of V3-V4 16S rRNA gene-based amplicon sequencing, full $16 \mathrm{~S}$ clone libraries, and 16S rRNA-targeted fluorescence in situ hybridization revealed that the genus Enterobacter dominated the glucose-fed SBR, and the genus Citrobacter in the xylose-fed SBR. Populations of Aeromonas, Clostridium, and Streptococcus coexisted in the glucose-fed CSTR, and Citrobacter and Lachnotalea in the xylose-fed CSTR.

The more diverse communities observed in the CSTRs could not be explained by sequential conversion of glucose to lactate and lactate to butyrate or acetate and ethanol, as no significant lactate uptake was observed. A wash-out experiment in the xylose CSTR showed that both populations washed-out simultaneously. This indicates a symbiotic relationship, which can explain high diversity in fermentative niches. Our follow-up investigations aim for understanding this relationship through a combination of metagenomics and physiological characterization of isolates. 


\title{
HUMAN INTESTINAL CORE MICROBIOME: CLUES FOR A MINIMAL MICROBIOME
}

\author{
SUDARSHAN A. SHETTY ${ }^{1}$ (SUDARSHAN.SHETTY@WUR.NL), \\ FLOOR HUGENHOLTZ ${ }^{1}$, LEO LAHTI ${ }^{1}$, HAUKE SMIDT ${ }^{1}$, WILLEM M. DE VOS VI, $^{1,2}$ \\ ${ }^{1}$ Laboratory of Microbiology, Wageningen University, Stippeneng 4, 6708 WE Wageningen, \\ The Netherlands \\ ${ }^{2}$ Department of Bacteriology and Immunology, Helsinki University, P.O. Box 21, \\ 00014 Helsinki, Finland \\ ${ }^{3}$ Research Programme Unit Immunobiology, Department of Veterinary Biosciences, \\ Helsinki University, P.O. Box 66, 00014 Helsinki, Finland
}

\begin{abstract}
The human gastrointestinal tract (GIT) is a complex ecosystem, and identifying a minimal microbiome can aid in understanding this ecosystem. Minimal microbiomes can assist in investigating ecological theories, such as invasion, resilience and species interactions. Hence, we characterised the phylogenetic core microbiota in the GIT of over 1000 healthy Western adults using the Human Intestinal Tract phylogenetic microarray (HITChip). In addition, we compared the genomes of representative core bacteria. Competition and complementarity between the bacteria in the core microbiome was calculated using a reverse ecology method. The GIT of $95 \%$ of the healthy adults shared 34 genus-like groups at a minimum detection threshold of $0.1 \%$ relative abundance (Shetty et al., 2017). The highest prevalence was observed for bacteria belonging to the order Clostridiales. Bacteria related to Faecalibacterium prausnitzii, Oscillospira guillermondii and Ruminococcus obeum were the top three taxa shared by all adults. Genomic analysis further revealed high diversity in gene content within the Firmicutes phylum while bacteria from the Bacteroidetes shared high similarity. A reverse ecology approach in combination with network statistics suggests a key role of Ruminococcus bromii and Akkermansia muciniphila in the GIT ecosystem. To further validate these predictions, we are using in-vitro methods to better understand underlying microbial interactions, providing the necessary basis for de novo assembly of defined synthetic bacterial communities.
\end{abstract}

\section{References}

Shetty, S. A., Hugenholtz, F., Lahti, L., Smidt, H., \& de Vos, W. M. (2017). Intestinal microbiome landscaping: insight in community assemblage and implications for microbial modulation strategies. FEMS Microbiology Reviews, 41(2), 182-199. 


\title{
CHARACTERISATION OF SMALL AND LARGE INTESTINAL MICROBIAL COMPOSITION IN WILD BOARS
}

\author{
ALEXANDER UMANETS ${ }^{1}$, IRATXE DIEZ DELGADO ${ }^{2}$, HAUKE SMIDT $^{1}$ \\ ${ }^{1}$ Laboratory of Microbiology, Wageningen University, The Netherlands \\ ${ }^{2}$ SaBio, Institute for Game and Wildlife Research, Spain
}

Improvement of production animals' health and reduction of drug use in livestock practice via modulation of the gut microbiota is a promising area of research. In search for microorganisms potentially useful for microbiota management in pig production and understanding of variation in microbiota composition between wild and domesticated animals we studied the microbial composition of wild boars, as the closet relatives of domestic pigs.

Intestinal samples from wild boars $(\mathrm{N}=22)$ were collected in the province of Ciudad Real, Spain, during hunting/population control events. Intestinal content samples from jejunum, ileum and colon were collected and frozen on dry ice within 2-3 hours after animals death. Microbial composition was determined using Next Generation Sequencing of PCR-amplified 16S ribosomal RNA gene.

Large and small intestinal microbiota were found to drastically differ, with much less pronounced differences between jejunum and ileum. Microbial composition of the colon is diverse (phylogenetic diversity $(P D)$ index $=5.9 \pm 0.7$ ) and dominated (relative abundance $>5 \%$ ) by an unassigned genus (UG) from the Ruminococcaceae family (24.2 $\pm 8.7 \%$ ), UG from the Christensenellaceae (7.8 $\pm 3.7 \%)$, UG from the Prevotellaceae $(7.5 \pm 4.4 \%)$ and an UG from the Bacteroidales order $(6.9 \pm 4.3 \%)$. As compared to the colon, jejunal and ileal microbiota were characterized by a lower alpha diversity ( $P D=1.5 \pm 0.4$ and $1.6 \pm 0.4$ respectively). Jejunal microbiota was enriched in members of Lactobacillus $(70.7 \pm 17.3 \%)$ with the second most abundant genus (Escherichia-Shigella) accounting only for $6.5 \pm 9.6 \%$. Ileal microbiota was characterized by a higher relative abundance of the genera Streptococcus and EscherichiaShigella (28.2 $\pm 19.8 \%$ and $22.5 \pm 12.9 \%$ respectively), and decrease of Lactobacillus $(21.9 \pm 11.7 \%)$ as compared to the jejunum.

Wild boars harbour largely undescribed intestinal microbiota featuring a range of yet unassigned genera without any cultured representative, belonging to families that have previously been associated with intestinal health, making the intestine of wild boars an attractive source in the quest for new beneficial microorganisms. 


\title{
THE HOST-MICROBIOTA INTERACTOME IN EARLY LIFE AND ITS ROLE IN GUT IMMUNE DEVELOPMENT
}

\author{
HUGO DE VRIES ${ }^{1}$ (HUGO.DEVRIES@WUR.NL) \\ HAUKE SMIDT ${ }^{2}$ (HAUKE.SMIDT@WUR.NL) \\ JERRY WELLS ${ }^{3}$ (JERRY.WELLS@WUR.NL) \\ ${ }^{1}$ Laboratory of Microbiology, Wageningen University \& Research, The Netherlands \\ 2 Laboratory of Microbiology, Wageningen University \& Research, The Netherlands \\ ${ }^{3}$ Host-Microbe Interactomics Group, Wageningen University \& Research, The Netherlands
}

\section{Introduction}

In order to develop more sustainable livestock production systems, the health status of production animals has to be improved. Pre- and probiotics are interesting candidates to enhance health by means of elevating immune development, stimulating growth of beneficial bacteria and by improving the intestinal barrier. Successful application of such dietary components can result in reduction of antibiotic usage, which will lower the pressure on the emergence of antibiotic resistant microbes.

The mucosal immune system is exposed to pathogens, dietary components and commensal bacteria and in order to maintain gut homeostasis must either produce an active immune response or must react with tolerance. The ability to react accurately is important, since a wrong decision can result in allergy or susceptibility to infectious diseases.

Aim

The aim of the project is to study dietary effects on microbiota composition in early life, identification of a healthy microbiome and determining its effect on host cells.

\section{Approach}

To study the microbiota composition development in early life, pig and chicken in vivo studies will be carried out during which faecal and intestinal samples will be taken. Microbiota composition analysis will be determined by Illumina HiSeq sequencing of PCR-amplified $16 \mathrm{~S}$ ribosomal RNA gene fragments.

To study the effect of dietary and microbial components on host cells, an in vitro assay will be developed that encompasses the growth of 3D organoids and organoid monolayers followed by stimulation. Subsequently, expression profiles of organoid cells are analysed for pro- and intiinflammatory responses. 


\section{Poster pitches \\ October 19, 2017 \\ $16.25-16.35$ hrs}




\title{
AUTONOMOUS PROCESSING OF A SINGLE CRISPR-RNA ARRAY BY CRISPR-CAS12A ENABLES SUCCESSFUL MULTIPLEX GENE EDITING
}

\author{
PRARTHANA MOHANRAJU ${ }^{1 *}$ (PRARTHANA.MOHANRAJU@WUR.NL), \\ BERND ZETSCHE ${ }^{2}$, MATTHIAS HEIDENREICH ${ }^{2 *}$, WEN Y. WU ${ }^{1}$, FENG ZHANG ${ }^{2}$, \\ JOHN VAN DER OOST ${ }^{1}$ \\ ${ }^{1}$ Laboratory of Microbiology, Department of Agrotechnology and Food Sciences, \\ Wageningen University, Dreijenplein 10,6703 HB Wageningen, The Netherlands \\ ${ }^{2}$ Broad Institute of MIT and Harvard, Cambridge, MA 02142 \\ *These authors contributed equally to this work.
}

\begin{abstract}
CRISPR-Cas systems that provide immunity against mobile genetic elements in prokaryotes are mechanically diverse. In the well-studied type II systems, RNase III cleaves precursor CRISPR RNA (pre-crRNA) that is base-paired with trans-activating crRNA (tracrRNA) in the presence of Cas9 to generate mature guides. The tracrRNA-crRNA duplex then guides Cas 9 to cleave target DNA. Here, we show that the type V-A Cas12a (formerly Cpf1) is a dual-nuclease that is specific to crRNA biogenesis and target DNA interference. We have confirmed in vitro that Cpf1 alone is sufficient for maturation of crRNAs. The CRISPR-Cas9 system has become a highly efficient site-directed genome editing technique. However, targeting of multiple genomic loci with Cas9 is limited by the need for multiple or large expression constructs. The ability of Cas12a to process its own pre-crRNA to mature crRNA guides can be used to simplify multiplexed genome editing. Using a single customized CRISPR array, we edit up to four genes in mammalian cells simultaneously.
\end{abstract}




\title{
SYNTROPHIC COCULTURES OF GEOBACTER SULFURREDUCENS AND SYNTROPHOBACTER FUMAROXIDANS GROWING ON PROPIONATE AND FE (III) OR A SOLID ELECTRODE AS ELECTRON ACCEPTORS
}

\author{
MONIR MOLLAEI ${ }^{1,2}$ (MONIR.MOLLAEI@WUR.NL), \\ TOM H.J.A. SLEUTELS ${ }^{2}$, ALFONS J.M. STAMS ${ }^{1,3}$, CAROLINE M. PLUGGE ${ }^{1,2}$ \\ ${ }^{1}$ Laboratory of Microbiology, Wageningen University and Research, Wageningen, \\ The Netherlands \\ ${ }^{2}$ Wetsus, European Centre of Excellence for Sustainable Water Technology, Leeuwarden, \\ The Netherlands \\ ${ }^{3}$ Center of Biological Engineering, University of Minho, Braga, Portugal
}

\begin{abstract}
A syntrophic coculture of the electroactive bacterium Geobacter sulfurreducens and the propionate-oxidising bacterium Syntrophobacter fumaroxidans was constructed with propionate as electron donor and $\mathrm{Fe}$ (III) as electron acceptor. The Fe (III)-reducing G. sulfurreducens use hydrogen, formate and acetate as the electron donor, but is unable to metabolise propionate. S. fumaroxidans on the other hand cannot use Fe (III) as electron acceptor. G. sulfurreducens was first grown in pure culture with single and different combinations of the electron donors i.e. acetate, formate and hydrogen, the products of propionate conversion by $S$. fumaroxidans. All single electron donors supported growth of G. sulfurreducens where $6.44 \pm 0.02,2.43 \pm 0.01$ and $1.63 \pm 0.41$ mole of Fe (II) was produced per mole of acetate, formate and hydrogen, respectively. When combinations of electron donors were applied, formate was always the preferred electron donor. Coculture results showed growth of these two bacteria with propionate and Fe (III) in batch cultures, where neither of bacteria was independently capable of such growth. The ratio of Fe (II) produced to propionate consumed was $12: 1$ on average which is close to the theoretical ratio of 14:1. To prove whether $S$. fumaroxidans and G. sulfurreducens can drive current production in a Microbial Electrolysis Cell (MEC), the coculture was applied in a MEC with propionate as substrate and the solid electrode at an anode potential of $-300 \mathrm{mV} \mathrm{vs} \mathrm{Ag} / \mathrm{AgCl}$ as electron acceptor. The results confirmed that the coculture was able to oxidize propionate and produce current density of $22 \mathrm{~A} / \mathrm{m}^{3}$, and $35 \%$ Coulombic efficiency.
\end{abstract}




\title{
IDENTIFICATION OF ARSENIC-OXIDIZING BACTERIA IN RAPID SAND FILTERS
}

\author{
M.J. OOSTERKAMP (M.J.OOSTERKAMP@TUDELFT.NL)*, \\ J. GUDE (J.C.J.GUDE@TUDELFT.NL)*, D. VAN HALEM \\ Section Sanitary Engineering, TU Delft, The Netherlands \\ *These authors contributed equally
}

\begin{abstract}
Arsenic can be present in groundwater from which drinking water is produced. It may have negative effects on human health such as arsenicosis and cardiovascular diseases ${ }^{1}$. Drinking water companies in the Netherlands currently aim to lower the level of arsenic in drinking water from 10 to $1 \mu \mathrm{g} / \mathrm{L}$. A possible strategy of stimulating arsenic removal to reach this lower level is biological oxidation. Biotic arsenic-oxidation has recently been proposed to occur in rapid sand filters ${ }^{2}$ and is likely important for arsenic removal from drinking water. The objective of the current work is to provide insight in biological arsenic-oxidation in rapid sand filters allowing near-complete arsenic removal from drinking water. Initial next generation sequencing of rapid sand filters revealed the presence of mainly Proteobacteria belonging to the Alpha- Beta- and Gammaproteobacteria and to Deltaproteobacteria in a minor extent ${ }^{2}$. Arsenic-oxidizing microorganisms broadly exhibit chemolithoautotrophic and heterotrophic metabolic strategies. Chemolithoautotrophic bacteria gain energy by using arsenic as the principal electron donor ${ }^{3}$ where heterotrophic bacteria oxidize arsenic chiefly as detoxification mechanism ${ }^{4}$. Alphaproteobacteria have been linked to chemolithoautotrophic arsenic-oxidizing bacteria and Beta- and Gammaproteobacteria to heterotrophic arsenic metabolism ${ }^{5}$. Further research involves next generation sequencing of rapid sand filters removing arsenic and challenged with manganese or ammonia. Interestingly, arsenic conversion and scavenging by manganese oxides produced in co-cultures of Arthrobacter QXT-31 and Sphingopyxis QXT-31 has been recently described ${ }^{6}$. The aim of this study is to identify bacteria involved in and related to arsenic oxidation in rapid sand filters. Results will be presented during the centennial symposium.
\end{abstract}

\section{References}

${ }^{1}$ Van Halem et al. 2009 Drink Water Eng Sci 2:29-34

${ }^{2}$ Gude et al. 2017 Water Research Under review

${ }^{3}$ Santini et al. 2000 Appl Environ Microbiol 66:92-97

${ }^{4}$ Tsai et al. 2009 Curr Opin Biotechnol 20:659-667

${ }^{5}$ Cavalca et al. 2013 Future Microbiol 8:753-768

${ }^{6}$ Liang et al. 2017 Microb Ecol DOI:10.1007/s00248-017-1008-9 


\title{
MICROBIAL SYSTEMS FOR CONVERSION OF SYNGAS TO BIO- BASED PRODUCTS
}

\author{
MARTIJN DIENDER ${ }^{1}$, ALFONS J.M. STAMS ${ }^{1,2}$ AND DIANA Z. SOUSA ${ }^{1}$ \\ ${ }^{1}$ Laboratory of Microbiology, Wageningen University, Wageningen, The Netherlands \\ ${ }^{2}$ Centre of Biological Engineering, University of Minho, Braga, Portugal
}

Synthesis gas, a mixture of $\mathrm{CO}, \mathrm{H}_{2}$, and $\mathrm{CO}_{2}$, can be created via gasification of any carbohydrate material. Fermentation of syngas by carboxydotrophic microbes allows for it to be converted into interesting bio-chemicals. Organisms involved in the fermentation of syngas use the $\mathrm{CO}$ or $\mathrm{H}_{2}$ in the gas as electron donor, fixating $\mathrm{CO}_{2}$ into the final end products. Currently acetate and ethanol are relatively well established products from syngas fermentation and there is interest to broaden the scope towards production of more complex products. However, genetic engineering of carboxydotrophic organisms and the knowledge of their metabolism is rather limited, making it difficult to 'create' strains producing these products.

A possible way to broaden the scope of products is via co-cultivation of microbes which can make use of each other's products. We established a co-cultivation of Clostridium autoethanogenum, a well-known carboxydotrophic acetogen, together with Clostridium kluyveri, a well characterized organism employing the reverse $\beta$-oxidation pathway. C. autoethanogenum uses the syngas to produce a mixture of acetate and ethanol. C. kluyveri subsequently uses these products to perform chain elongation. This results in a co-culture producing a mixture of $\mathrm{C} 4$ and $\mathrm{C} 6$ acids and alcohols using carbon monoxide as a sole substrate.

This co-culture poses an interesting way for production of more complex and valuable products from syngas. Basic characterization of these co-cultures has been done and currently the research focus lies on how the species interact with each other and how environmental factors influence their production patterns and metabolism. 


\title{
ASSESSMENT OF READY BIODEGRADABILITY OF ALKYL POLYGLUCOSIDES USING PURE AND MIXED BACTERIAL CULTURES
}

\author{
R. GEERTS ${ }^{1,2}$ (ROY.GEERTS@AKZONOBEL.COM), \\ C.G. VAN GINKEL ${ }^{1}$, C.M. PLUGGE ${ }^{2}$ \\ ${ }^{1}$ AkzoNobel Chemicals B.V., The Netherlands \\ ${ }^{2}$ Laboratory of Microbiology, Wageningen University, The Netherlands
}

Alkyl polyglucosides (APGs) are non-ionic surfactants consisting of a lipophilic alkyl chain and a hydrophilic carbohydrate moiety linked through a glycosidic bond. Industrially produced APGs are multiconstituent substances primarily due to the number of glucose units, and the three types of isomers present in the carbohydrate moiety (stereoisomerism, positional isomerism and ring isomerism). One by one testing of the ready biodegradability of individual constituents present in APGs is difficult or even impossible due to the large number of constituents and/or the unavailability of pure constituents. As an alternative for testing individual compounds, read-across may be used to assess the ready biodegradability of APG constituents.

Ready biodegradability of industrially produced hexyl glucoside and 2-ethylhexyl glucoside was demonstrated in Closed Bottle tests inoculated with river water. Read-across of ready biodegradability for APG constituents was carried out using APG-degrading bacteria isolated from river water. These bacteria were isolated by selection on prevalence using hexyl glucoside, 2-ethylhexyl glucoside, hexyl- $\beta$-maltoside, glucose, and maltose as sole sources of carbon and energy. The first step in the biodegradation of APGs by the isolates is the cleavage of the glycosidic bond between the alkyl chain and the (oligo)-saccharide moiety. Stoichiometric formation of hexanol and 2-ethylhexanol demonstrated that all constituent of the APGs are degraded by the isolates. Glycosidase activity constitutively present in two strains was mainly cellular bound and the $\beta$-glycosidase activity being higher than the a-glycosidase activity. The formed (oligo)-saccharides were biodegraded by 70 to $80 \%$ by all isolated strains and completely removed by microorganisms present in river water.

Read-across of ready biodegradability for all constituents of the APGs studied is justified based on: the broad substrate specificity of glucosidases in the first biodegradation step, the stoichiometric formation of alcohols, and the biodegradation of the transiently formed alcohols, glucose, disaccharides and probably the higher oligosaccharides. 


\title{
ENRICHMENT OF SULFIDOGENIC BACTERIA FROM THE HUMAN INTESTINAL TRACT
}

\author{
YUAN FENG ${ }^{1}$ (YUAN.FENG@WUR.NL), \\ ALFONS J.M. STAMS ${ }^{1,2}$, WILLEM M. DE $\operatorname{VOS}^{1,3}$ AND IRENE S 'ANCHEZ-ANDREA ${ }^{1}$ \\ ${ }^{1}$ Laboratory of Microbiology, Wageningen University, Stippeneng 4, 6708 WE Wageningen, \\ The Netherlands \\ ${ }^{2}$ IBB - Institute for Biotechnology and Bioengineering, Centre of Biological Engineering, \\ University of Minho,Campus de Gualtar, 4710-057, Braga, Portugal \\ ${ }^{3}$ Department of Bacteriology and Immunology, Faculty of Medicine University of Helsinki, \\ 00014 Helsinki, Finland
}

\begin{abstract}
Hydrogen sulfide is formed in the human intestinal tract as the end product of the anaerobic microbial degradation of sulfur compounds present in mucus, bile or proteins. Since human gut microbial sulfur metabolism has been poorly characterized, we aimed to identify and isolate the microorganisms involved in sulfide formation. Fresh fecal samples from one healthy donor and one diagnosed with irritable bowel syndrome were used as inocula for enrichments that were supplemented with sulfate or sulfite as electron acceptors in combination with different electron donors. After two transfers, cultures with high sulfide production were selected and the phylogenetic composition of the enriched microbial communities was determined. Sulfite respiration and cysteine degradation were the dominant sulfidogenic processes, and the most abundant bacteria enriched belonged to Bilophila and Clostridium cluster XIVa. Different isolates were obtained and remarkably included a novel sulfite reducer, designated strain 2C. Strain $2 \mathrm{C}$ belongs to the Veillonellaceae family of Firmicutes phylum and showed limited (91\%) 16S rRNA gene sequence similarity with that of known Sporomusa species and hence may represent a novel genus. This study indicates that bacteria that utilize sulfite and organic sulfur compounds rather than merely sulfate are relevant for human intestinal sulfur metabolism.
\end{abstract}




\title{
ORGANIC ACID PRODUCTION FROM STARCHY WASTE BY RUMEN DERIVED MICROBIAL COMMUNITIES
}

\author{
SUSAKUL PALAKAWONG NA AYUDTHAYA ${ }^{1,2^{*}}$ \\ (SUSAKUL.PALAKAWONGNAAYUDTHAYA@WUR.NL), \\ ANTONIUS H.P. VAN DE WEIJER ${ }^{1}$, ANTONIE H. VAN GELDER ${ }^{1}$, ALFONS J.M. STAMS ${ }^{1,3}$, \\ WILLEM M. DE $\operatorname{VOS}^{1,4}$, AND CAROLINE M. PLUGGE ${ }^{1,5}$ \\ ${ }^{1}$ Laboratory of Microbiology, Wageningen University \& Research, Stippeneng 4, \\ 6708 WE Wageningen, The Netherlands \\ ${ }^{2}$ Thailand Institute of Scientific and Technological Research, $35 \mathrm{Mu}$ 3, Khlong Ha, \\ Amphoe Khlong Luang, Pathum Thani 12120 Thailand \\ ${ }^{3}$ CEB-Centre of Biological Engineering, University of Minho, Campus de Gualtar, \\ 4710-057 Braga, Portugal \\ ${ }^{4}$ RPU Immunology, Department of Bacteriology and Immunology, University of Helsinki, \\ Haartmaninkatu 3, FIN-00014 Helsinki, Finland \\ ${ }^{5}$ Wetsus, European Centre of Excellence for Sustainable Water Technology, Oostergoweg 9, \\ 8911 MA Leeuwarden, The Netherlands
}

\begin{abstract}
Converting organic waste to energy carriers and valuable products such as organic acids (OA) using microbial fermentation is one of the sustainable options of renewable energy. Substrate and inoculum are important factors in optimizing the fermentation. In this study, we investigated organic acid production and microbial composition shift during the fermentation of starchy (potato) waste using two different sources of rumen fluid, obtained from fistulated cows in the Netherlands and Thailand as inoculum. The bacterial community profiles of both reactors were analysed using 16S rRNA amplicon pyrosequencing and the relationship between the communities and the organic acid production profiles were determined. The results showed that Streptococcus spp. are important for fast lactate (up to $250 \mathrm{mM}$ ) production during the first stage ( 0 - 1 day) in both reactors. Lactate was then the substrate for secondary fermentation to produce acetate, butyrate, and propionate. OA production profile and bacterial composition of both reactors were different in the second part of the fermentation. At day 8 , in the reactor with a Dutch rumen inoculum, butyrate $(140 \mathrm{mM})$ and acetate $(100 \mathrm{mM})$ were the main products that correlated with increases in relative abundances of Parabacteroides, Sporanaerobacter, Helicobacteraceae, Peptostreptococcaceae, and Porphyromonadaceae. In the reactor with the Thai rumen inoculum, acetate $(190 \mathrm{mM})$, propionate $(120 \mathrm{mM})$, and butyrate $(70 \mathrm{mM})$ were produced, which correlated with increases in relative abundances of Bacteroides, Dysgonomonas, Eubacterium, Enterobacteraceae, and Porphyromonadaceae. In summary, starchy waste is very suitable as alternative source for organic acid production, especially lactate, using rumen fluid as an inoculum. OA production was dependent on the inoculum source and Streptococcus spp. ( $86 \%$ average relative abundance) played an important role in lactate production in the first stage of the fermentation process.
\end{abstract}




\section{SYNTROPHIC ACETATE OXIDATION AT HALOALKALINE CONDITIONS}

PEER H.A.TIMMERS ${ }^{1,2}$ (PEER.TIMMERS@WUR.NL/PEER.TIMMERS@WETSUS.NL), C. VAVOURAKIS ${ }^{3}$, G. MUYZER ${ }^{3}$, D. SOROKIN ${ }^{4,5}$, ALFONS J.M. STAMS ${ }^{1,6}$, CAROLINE M. PLUGGE ${ }^{1,2}$

${ }^{1}$ Laboratory of Microbiology, Wageningen University, Stippeneng 4, 6708 WE Wageningen, The Netherlands

${ }^{2}$ Wetsus, European Centre of Excellence for Sustainable Water Technology, Oostergoweg 9, 8911 MA Leeuwarden, The Netherlands

${ }^{3}$ Microbial Systems Ecology, Department of Aquatic Microbiology, Institute for Biodiversity and Ecosystem Dynamics, University of Amsterdam, Amsterdam, The Netherlands

${ }^{4}$ Research Centre of Biotechnology, Winogradsky Institute of Microbiology, Russian Academy of Sciences, Moscow, Russia

${ }^{5}$ Department of Biotechnology, Delft University of Technology, Delft, The Netherlands

${ }^{6}$ Centre of Biological Engineering, University of Minho, Campus de Gualtar, 4710-057 Braga, Portugal

Syntrophic acetate oxidation (SAO) is a process where syntrophic acetate-oxidizing bacteria (SAOB) convert acetate to form $\mathrm{CO}_{2}$ and reducing equivalents. These reducing equivalents are utilized by a syntrophic partner which in most cases is a hydrogenotrophic methanogen or sulfate-reducing bacterium. Until now, SAO has been found to be dominant over aceticlastic methanogenesis under extreme conditions such as high ammonia or high fatty acid concentrations and high temperatures. Here, we investigated the pathway of acetate utilization and the mechanism of SAO in haloalkaline soda lakes that have extremely high soda concentrations and $\mathrm{pH}$. Enrichment cultures of SAOB together with a methanogenic (M-SAO) or sulfate-reducing (S-SAO) syntrophic partner showed that hydrogen is an important interspecies electron carrier. Metagenome analysis of the M-SAO enrichment culture showed that "Candidatus Syntrophonatronum acetioxidans" is the dominant SAOB, and its genome contained all genes necessary for operating the Wood Ljungdahl pathway in reverse. The genome contained an $F_{1} F_{0}$ ATP synthase that uses a proton motive force (pmf), but it also contained an energy conserving hydrogenase (Ehb) that pumps out $\mathrm{Na}^{+}$instead of $\mathrm{H}^{+}$while consuming intracellular $\mathrm{H}^{+}$to form $\mathrm{H}_{2}$. This simultaneously generates a pmf while pumping out $\mathrm{Na}^{+}$, which is probably an adaptation to the high $\mathrm{Na}^{+}$concentrations in soda lakes. Highthroughput 16S rRNA gene sequencing results of five different soda lake sediments showed that "Candidatus Syntrophonatronum acetioxidans" related sequences were up to $1.3 \%$ of all reads and the overarching Syntrophomonadaceae family was highly abundant (up to $24.5 \%$ of total reads). Known aceticlastic methanogenic genera were mostly absent. These results show that SAO, and syntrophy in general, is an important process in soda lakes which is probably a result of the extreme haloalkaline conditions. 


\title{
ENRICHMENT AND ISOLATION OF NOVEL POLYSACCHARIDE- DEGRADING ANAEROBES FROM ABYSSAL BLACK SEA SEDIMENT
}

\author{
D.M. VAN VLIET ${ }^{1,2}$ (DAAN.VANVLIET@WUR.NL), \\ A.J.M. STAMS ${ }^{1,2,3}$, L. VILLANUEVA ${ }^{2,4}$, I. SÁNCHEZ-ANDREA ${ }^{1,2}$ \\ ${ }^{1}$ Laboratory of Microbiology, Wageningen University, Wageningen, The Netherlands. \\ ${ }^{2}$ Soehngen Institute of Anaerobic Microbiology (SIAM), Nijmegen, The Netherlands \\ ${ }^{3}$ Centre of Biological Engineering, University of Minho, Braga, Portugal \\ ${ }^{4}$ Department of Marine Organic Biogeochemistry, Royal Netherlands Institute for Sea Research
}

(NIOZ), 't Horntje, Texel, The Netherlands

\begin{abstract}
Marine anaerobes play an important role in the global carbon cycle. This includes the degradation of organic matter by hydrolytic and fermentative marine anaerobes, of which ecologically relevant isolates are lacking. This lack could stem from the use of monomeric substrates in anaerobic cultivation rather than the recalcitrant polymeric substrates available to marine anaerobes in situ. We tested this hypothesis by using environmentally representative cultivation conditions with polysaccharides as substrates. Enrichment cultures were set up with mineral marine medium and low concentrations $(2.5 \mathrm{mM}$ monomer equivalent) of one of several polysaccharides. The cultures were inoculated with anoxic Black Sea sediment from the abyssal plain at $2100 \mathrm{~m}$ depth and incubated at $15^{\circ} \mathrm{C}$ (in situ $\mathrm{T}=8^{\circ} \mathrm{C}$ ). Various microbial clades without cultured representatives were enriched, indicating a potential hydrolytic and/or fermentative metabolism. We isolated hydrolytic strains of Kiritimatiellaeota (formerly Verrucomicrobia subdivision five) clade R76-B128, which is ubiquitous in anoxic marine waters, including the Black Sea. The only isolate of this clade is Kiritimatiella glycovorans, which is a halophilic bacterium that does not grow on polysaccharides. The $16 \mathrm{~S}$ rRNA genes of our isolates form two phylogenetic clusters that share $94 \%$ identity with each other, and $83-84 \%$ identity with the $16 \mathrm{~S}$ rRNA gene of $K$. glycovorans. According to taxonomic threshold values, this is strong evidence that our isolates represent two novel genera within a novel family of the Kiritimatiellaeota. Ongoing work on these strains includes characterization, genomics, and comparative proteomics to identify hydrolytic pathways.
\end{abstract}




\title{
TRICHOCOCCUS SPECIES AS CATALYSTS FOR BIOTECHNOLOGICAL PRODUCTION OF 1,3-PROPANEDIOL
}

\author{
NIKOLAOS STREPIS ${ }^{1,2}$ (NIKOLAOS.STREPIS@WUR.NL), \\ JASPER KOEHORST ${ }^{2}$, \\ HENRY BENAVIDES NARANJO ${ }^{1}$ (HENRY.NARANJO@WUR.NL), \\ PETER SCHAAP ${ }^{2}$ (PETER.SCHAAP@WUR.NL), \\ ALFONS J.M. STAMS ${ }^{1,3}$ (FONS.STAMS@WUR.NL), \\ DIANA Z. SOUSA ${ }^{1}$ (DIANA.SOUSA@WUR.NL) \\ ${ }^{1}$ Laboratory of Microbiology, Wageningen University, Stippeneng 4, 6708 WE Wageningen, \\ The Netherlands \\ ${ }^{2}$ Laboratory of Systems and Synthetic Biology, Wageningen University, Stippeneng 4, \\ 6708 WE Wageningen, The Netherlands \\ ${ }^{3}$ Centre of Biological Engineering, University of Minho, 4710-057 Braga, Portugal
}

\begin{abstract}
Nowadays, large amounts of waste glycerol are generated as a major byproduct from biodiesel industry. Glycerol can be converted to 1,3-propanediol (1,3-PDO), a building block for the synthesis of polyester and biodegradable plastic fibers. We have previously isolated a novel Trichococcus species, strain ES5, which is capable to anaerobically produce 1,3-PDO from glycerol, an ability it shares with Trichococcus pasteurii. In this work, we analyzed and compared the genomes of these two bacteria and studied the production of 1,3-PDO by cultures of $T$. pasteurii and strain ES5 under different conditions. A continuous bioreactor system was established for achieving production of 1,3-PDO. A complete operon structure composed by 16 genes related to 1,3-PDO metabolism could be identified in the genomes of T. pasteurii and strain ES5. This genomic synteny contains the two essential genes for glycerol conversion to 1,3-PDO, glycerol dehydratase and 1,3-propanediol dehydrogenase, and additional genes for glycerol uptake and regulation factors of glycerol metabolism. The metabolic trait of 1,3-production was in silico analysed for the complete bacterial kingdom and genes were identified with an essential or accessory influence for the trait.

Bacteria with fewer genes were underperforming when tested in vitro for production 1,3-PDO. Furthermore, both Trichococcus strains could grow on glycerol in a broad range of temperatures, from 4 to $400 \mathrm{C}$ and strain ES5 achieved growth in 0oC. Such physiological properties may be advantageous for biotechnological production. Tolerance to high salinity may be an additional strength of Trichococcus species as a candidate for the biotechnological production of 1,3-PDO.
\end{abstract}

\section{Keywords}

glycerol; fermentation; 1,3-propanediol; low temperature; high-salinity; genome comparison 


\title{
MEMBRANE CLEANING VIA BACTERIAL DISPERSION: STOP- FLUSH AND GO!
}

\author{
H.J. DE VRIES ${ }^{1,2}$ (RIK.DEVRIES@WETSUS.NL), \\ E. KLEIBUSH ${ }^{1,3}$, P. VAN DEN BRINK ${ }^{2}$, C.M. PLUGGE \\ ${ }^{1}$ Laboratory of Microbiology, Wageningen University, Stippeneng 4, 6708 WE Wageningen, \\ The Netherlands \\ ${ }^{2}$ Wetsus, European Centre of Excellence for Sustainable Water Technology, Oostergoweg 9, \\ 8911 MA Leeuwarden, The Netherlands \\ ${ }^{3}$ University Duisburg-Essen, Universitaetsstrasse 5, 45141 Essen, Germany
}

\begin{abstract}
The performance of membranes used for clean water production deteriorates when material accumulates on the membrane surface, causing fouling. To recover membrane performance, membranes need frequent cleaning. For spiral-wound membranes, not many options exist for fouling control. Spiral wound membranes are cleaned using alkaline and acidic solutions, but this strategy has major drawbacks, and alternative anti-fouling strategies are therefore highly desirable. Biological fouling is one of the most frequent observed fouling types and the biofilm embedded bacteria often resist the alkaline and acidic cleaning agents. However, bacteria may actively dissociate from the biofilm and become planktonic. This process is known as (bacterial) dispersion and is induced under certain stress conditions via cell-lysis, secretion of proteases or increased motility. Although this is an interesting alternative for biofilm removal and biofouling control, not much is known about how multispecies biofilms behave towards the environmental cues that are known to induce dispersion in single-species biofilms. For instance, when single-species biofilms are grown under flow conditions, dispersion can simply be induced by starvation and oxygen depletion via flow cessation. In this study, multispecies biofilms were grown on microfiltration membranes in membrane fouling simulators dosed with nutrients to accelerate biofilm formation. Operation was ceased after 5 days, for three specific time-periods, before the membranes were flushed. Important outcomes of this study are: (I) flow cessation does not per se induce dispersion in multispecies biofilms, (II) repeated stopping and starting of the flow increases the resistance of the biofilm towards membrane flushing. The results presented here are of fundamental interest, and have practical implications, because they suggest that the commonly observed loss of cleaning efficiency might simply be due to the evolving resistance of the biofilm against flushing events.
\end{abstract}




\section{Poster presentations Microbial ecology}

Presented in alfabetical order 


\title{
RELATING MICROBIAL COMMUNITY TO IN SITU NATURAL ATTENUATION OF MICROPOLLUTANTS
}

\author{
NORA B. SUTTON ${ }^{1}$ (NORA.SUTTON@WUR.NL), \\ JAVIER RAMIRO-GARCIA ${ }^{2}$, \\ SIAVASH ATASHGAHI ${ }^{2}$ (SIAVASH.ATASHGAHI@WUR.NL), \\ HAUKE SMIDT ${ }^{2}$ (HAUKE.SMIDT@WUR.NL), \\ EDOARDO SACCENTI ${ }^{3}$ (EDOARDO.SACCENTI@WUR.NL), \\ MARIA SUAREZ-DIEZ ${ }^{3}$ (MARIA.SUAREZDIEZ@WUR.NL), \\ ALETTE A.M. LANGENHOFF ${ }^{1}$ (ALETTE.LANGENHOFF@WUR.NL), \\ HUUB H.M. RIJNAARTS ${ }^{1}$ (HUUB.RIJNAARTS@WUR.NL) \\ ${ }^{1}$ Department of Environmental Technology, Wageningen University and Research, \\ P.O. Box 17, 6700 AA Wageningen, The Netherlands \\ ${ }^{2}$ Wageningen University \& Research, Laboratory of Microbiology, Stippeneng 4, \\ 6708 WE Wageningen, The Netherlands \\ ${ }^{3}$ Laboratory of Systems and Synthetic Biology, Wageningen University and Research, \\ P.O. Box 8033, 6700 EJ Wageningen, The Netherlands
}

\begin{abstract}
The presence of low concentrations of hydrophilic, organic emerging contaminants pose a threat to the quality of groundwater resources utilized for drinking water purposes. While extensive monitoring of the chemical composition of groundwater has revealed the presence of a variety of organic contaminants, relatively little is known about the natural attenuation of these compounds in situ. Biodegradation has been shown to be an effective transformation processes for a wide variety of emerging contaminants. However, it remains difficult to translate these results to in situ degradation in (usually) oxygen-depleted, oligotrophic groundwater with heterogeneous distribution and low emerging contaminant concentrations.
\end{abstract}

Research was performed to gain insight on biodegradation of emerging contaminants in groundwater by examining microbial community composition and geochemistry. Groundwater samples collected at discreet depths ranging from 12 to $55 \mathrm{~m}$ in two monitoring wells were chemically analyzed to determine groundwater chemistry and contaminant distribution. Additionally, samples were analysed for microbial community composition by sequencing of a PCR-amplified fragment of the 16S rRNA gene.

Results indicate a distinct difference in both groundwater composition and microbial community diversity between wells and in depth. The groundwater profiles demonstrate the heterogeneity of subsurface geochemistry, highlighting the fact that contaminated water travels through a variety of geochemical gradients between its source and extraction for drinking water. In addition to emerging contaminants, differences in the abundance of electron acceptors, concentration of nutrients, and presence of electron donors such as dissolved organic compound (DOC) seem to play important roles in microbial community composition and in situ biodegradation potential. Redundancy analysis of groundwater geochemical characteristics, emerging contaminant concentrations, and microbial community diversity indicates a negative correlation between the availability of electron acceptors (nitrate and sulfate) and the presence of contaminants. In contrast, dissolved organic carbon correlated with the presence of organic contaminants.

Overall, the results presented here aid in better understanding the geochemical and microbial factors affecting emerging contaminant biodegradation in situ. 


\title{
BIOLOGICAL CONTROL OF DIFFERENT FUNGAL PATHOGENS BY GANODERMA LUCIDUM
}

MIRZA NABEEL BAIG, AHMAD ALI SHAHID, \& MUHAMMAD ALI*

Institute of Agricultural Sciences, University of the Punjab, Lahore, Pakistan

\begin{abstract}
Five different isolates of the medicinal mushroom Ganoderma lucidum (GL-1, GL-2, GL-3, GL-4, GL-5) were collected from different places within and surrounding Lahore, Pakistan, to study the antifungal potential of their bioactive compounds. After studying morphology, different concentrations of mushroom extracts were prepared in methanol and water using a Soxhlet extractor. Different cultures of fungal pathogens were acquired from the First Fungal Culture Bank of Pakistan, University of the Punjab, Lahore. The antimicrobial potential of five G. lucidum samples against five fungal pathogens (Fusarium oxysporum, Aspergillus niger, A. flavus, Penicillium sp., and Alternaria alternata) was observed. The lowest biomass reduction ( $7 \%$ ) was observed in $1 \%$ and $2 \%$ concentrations of a methanolic extract and $6 \%$ in the case of a water extract. Major inhibition was observed using higher concentrations of the methanolic extract ( $3 \%$ and $4 \%$ ). These extracts significantly suppressed fungal biomass up to $38 \%$ and $56 \%$ for $A$. niger, $47 \%$ for $A$. flavus, $58 \%$ for Penicillium sp., $46 \%$ for $A$. alternaria, and $45 \%$ for $F$. oxysporum as compared to the control. It was concluded from these studies that methanolic extracts of $G$. lucidum showed better activity against all plant fungal pathogens when compared to the water extracts.
\end{abstract}

KEYWORDS: antifungal, Ganoderma lucidum, medicinal mushrooms, methanolic extract, Pathogenic fungi. 


\title{
GENETIC AND PHENOTYPIC VARIATION BETWEEN TWO PHYLOGENETIC DISTINCT CLUSTERS OF CLOSTRIDIUM PERFRINGENS
}

\author{
M. CALLAGHAN ${ }^{1}$ (MATTHEW.CALLAGHAN@NIZO.COM),
} J. BOEKHORST' ${ }^{2}$, M.H.J. WELLS-BENNIK ${ }^{3}$

${ }^{1}$ NIZO, The Netherlands; Host-Microbe Interactomics, Wageningen University \& Research, The Netherlands

${ }^{2} \mathrm{NIZO}$, The Netherlands

${ }^{3}$ NIZO, The Netherlands

Clostridium perfringens is an anaerobic spore-forming bacteria, and a major cause of food poisoning worldwide. Its ability to form spores enables the bacterium to survive harsher conditions than it would normally be able to as a vegetative cell. For example, spores of C. perfringens can survive high temperatures, high osmotic stress, extreme $\mathrm{pH}$, UV radiation, and exposure to chemicals, such as food preservatives and disinfectants.

The ability of $C$. perfringens spores to survive such environments correlates with the presence of the Clostridium perfringens enterotoxin-encoding gene cpe and its location within the genome. Strains with a chromosomal variant (C-cpe) often have higher resistance to such environments, compared to strains without the gene (non-cpe) or strains with a plasmid-based variant (P-cpe). Strains of different cpe locations have recently been shown to form two distinct phylogenetic clusters, and it is suggested that these clusters demonstrate preference for different environmental niches.

Using gene-trait matching on 24 genome sequenced $C$. perfringens strains, we have identified a range of genes that may be factors in the observed differences in spore resistance and niche adaptation, based on spore heat resistance and phylogenetic clustering. These genes cover a wide range of predicted functions, including: transport, carbon metabolism, regulators, energy generations, cell wall synthesis, host molecule metabolism, and sporulation/germinationassociated genes, including PrkC and AgrBCD homologues. In addition, we have tested the metabolic capabilities of the strains, to improve our understanding of environments that C. perfringens can inhabit.

Future research will involve mutant generation, using ClosTron and CRISPR technology, and subsequent phenotypic characterisation. This work will provide greater knowledge into heterogeneity of $\mathrm{C}$. perfringens strains regarding spore properties and niche adaptation. 


\title{
BOOLEAN ANALYSIS REVEALS SYSTEMATIC INTERACTIONS AMONG LOW-ABUNDANCE SPECIES IN THE HUMAN GUT MICROBIOME
}

\author{
JENS CHRISTIAN CLAUSSEN ${ }^{1}$ (J.CLAUSSEN@JACOBS-UNIVERSITY.DE), \\ JURGITA SKIECEVICIENE ${ }^{2,3}$, JUN WANG ${ }^{3}$, PHILIPP RAUSCH ${ }^{3,4}$, TOM H. KARLSEN $^{5,6}$, \\ WOLFGANG LIEB ${ }^{7}$, JOHN F. BAINES ${ }^{3,4}$, ANDRE FRANKE ${ }^{2}$, AND MARC-THORSTEN HÜTT ${ }^{1}$ \\ ${ }^{1}$ Computational Systems Biology, Jacobs University Bremen \\ ${ }^{2}$ Institute for Digestive Research, Lithuanian U of Health Sciences, Kaunas, Lithuania \\ ${ }^{3}$ Institute for Experimental Medicine, Christian Albrechts University Kiel, Germany \\ ${ }^{4}$ Max Planck Institute for Evolutionary Biology, Plön, Germany \\ ${ }^{5}$ Institute for Clinical Medicine, University of Oslo, Norway \\ ${ }^{6}$ Norwegian PSC Research Center, Oslo University Rikshospitalet, Norway \\ ${ }^{7}$ Third Institute of Epidemology, Christian Albrechts University Kiel, Germany
}

The analysis of microbiome compositions in the human gut has gained increasing interest due to the broader availability of data and functional databases and substantial progress in data analysis methods, but also due to the high relevance of the microbiome in human health and disease. While most analyses infer interactions among highly abundant species, the large number of low-abundance species has received less attention. In [J.C.Claussen et al., Plos Comp. Biol, in print, 2017] we present a novel analysis method based on Boolean operations applied to microbial co-occurrence patterns. We calibrate our approach with simulated data based on a dynamical Boolean network model from which we interpret the statistics of attractor states as a theoretical proxy for microbiome composition. We show that for given fractions of synergistic and competitive interactions in the model our Boolean abundance analysis can reliably detect these interactions. Analyzing a novel data set of 822 microbiome compositions of the human gut, we find a large number of highly significant synergistic interactions among these low-abundance species, forming a connected network, and a few isolated competitive interactions. 


\title{
DIVERSITY OF SPONGE-ASSOCIATED BACTERIA CULTURED TO DATE AND INFLUENCE OF CULTURE CONDITIONS
}

\author{
T.T.H. DAT ${ }^{1,2}$ (HUUDAT.TON@WUR.NL), \\ G. STEINERT ${ }^{2}$, N.T.K. CUC ${ }^{3}$, H. SMIDT ${ }^{2}$, \\ D. SIPKEMA ${ }^{2}$ (DETMER.SIPKEMA@WUR.NL) \\ ${ }^{1}$ Mientrung Institute for Scientific Research, Vietnam Academy of Science and Technology, \\ Vietnam \\ ${ }^{2}$ Laboratory of Microbiology, Wageningen University \& Research, Wageningen, \\ The Netherlands \\ ${ }^{3}$ Institute of Marine Biochemistry, Vietnam Academy of Science and Technology, Vietnam
}

\begin{abstract}
Marine sponges are the most ancient animals and harbour diverse microbial communities. However, only a minor fraction of these bacteria has been cultured in vitro to date. We reviewed 4263 publicly deposited 16S rRNA gene sequences of cultured bacteria from sponges. Based on this data we assessed the diversity of bacteria cultured to date as well as the influence of culture conditions on the composition of the cultured fraction. Representatives from 11 bacterial phyla have so far been cultivated from sponges, including Proteobacteria, Actinobacteria, Firmicutes, Bacteroidetes, Planctomycetes, Cyanobacteria, Verrucomicrobia, Acidobacteria, Chlorobi, Chloroflexi, and Lentisphaerae. Among these, the most frequently cultured phyla were Proteobacteria (55.9\%), Actinobacteria (21.5\%), Firmicutes (15.2\%), and Bacteroidetes (5.1\%). At the genus level, the most frequently retrieved taxa included Pseudovibrio (15.6\%), Bacillus (9.6\%), Streptomyces $(7.4 \%)$, followed by Vibrio $(6.0 \%)$ and Ruegeria (5.7\%). Furthermore, our analysis supported the notion that culture conditions strongly affected composition and relative abundance of cultured bacteria. While some taxa were independently isolated from a set of different conditions (e.g., Pseudovibrio or Bacillus), some taxa were only isolated when using specific cultivation conditions (e.g., Salinispora from agar plates, or Synechococcus via liquid cultivation). Moreover, several isolated genera (e.g., Pseudovibrio, Bacillus, or Ruegeria) were retrieved at a range of different temperatures and carbon concentrations. In contrast, representatives of several genera belonging to the Actinobacteria preferred media with moderate or high carbon content, whereas cyanobacterial isolates could only be cultured on low carbon content media. In conclusion, the meta-analysis presented here reinforces the notion that there is no golden bullet for cultivation of marine sponge-associated microorganisms. Rather, high throughput approaches comprising a range of different, complementary media and cultivation platforms can provide for more comprehensive coverage of sponge symbionts in culture collections as a starting point for unlocking their full potential as producers of novel bioactive metabolites.
\end{abstract}




\title{
ENZYMATIC DEGRADATION OF HUMAN MILK OLIGOSACCHARIDES BY AKKERMANSIA MUCINIPHILA
}

\author{
J. ELZINGA (JANNEKE.ELZINGA@WUR.NL), \\ I. KOSTOPOULOS, N. OTTMAN, \\ W.M. DE VOS, J. KNOL, C. BELZER
}

Laboratory of Microbiology, Wageningen University, The Netherlands

\begin{abstract}
The composition of the gut microbiota in early life is shaped by many factors and has been suggested to influence health later in life. Human Milk Oligosaccharides (HMOs) are the main source of dietary glycans during the first months of a baby's life. These carbohydrates selectively stimulate the growth of beneficial bacteria in the infant intestine. Because O-linked glycans of human milk and human mucus are structurally similar, it is predicted that some bacterial species known to utilize intestinal mucin, are also able to degrade HMOs. Akkermansia muciniphila is an example of a mucin-degrading intestinal inhabitant. Its presence has been associated with a healthy mucosa. Because of its outstanding mucindegrading capabilities and its colonization during the first year of life, we hypothesized that $A$. muciniphila is able to degrade HMOs. We demonstrated the growth of $A$. muciniphila in human breast milk, albeit more slowly compared to conditions in mucin. Proteomics revealed that growth on human milk was accompanied by the expression of key mucin-degrading enzymes of $A$. muciniphila, such as a-L-fucosidases, sialidases and $\beta$-galactosidases. A selection of these enzymes were expressed in E.coli, purified and used to determine their ability to degrade HMOs in vitro. Several enzymes were able to cleave HMO-structures efficiently. The ability of A. muciniphila to degrade HMOs could provide further insight in its survival during early life colonization and its contribution to a developing mucosal layer.
\end{abstract}




\title{
'GENOME' EXPLORATION OF MEMBERS OF THE FLAVOBACTERIACEAE FAMILY ISOLATED FROM DIFFERENT SPONGE SPECIES: A COMPARATIVE GENOMICS APPROACH
}

\author{
A. GAVRIILIDOU ${ }^{1}$ (ASIMENIA.GAVRIILIDOU@WUR.NL), \\ J. GUTLEBEN ${ }^{1}$, D. VERSLUIS ${ }^{1}$, C. INGHAM ${ }^{2}$, H. SMIDT ${ }^{1}$, D. SIPKEMA ${ }^{1}$ \\ ${ }^{1}$ Laboratory of Microbiology, Wageningen University \& Research, Wageningen, \\ The Netherlands \\ ${ }^{2}$ Hoekmine BV, Utrecht, The Netherlands
}

\begin{abstract}
Marine sponges and their microbial symbionts have garnered great interest in the last decades, due to both their complex symbiosis and their ability to produce metabolically interesting molecules. The family Flavobacteriaceae contains many genera and species isolated from marine sources (also denoted as the "marine clade") that are often among the most abundant organisms in the ocean. As rich sources of enzymes involved in the digestion of carbohydrates and proteins, flavobacteria hold great potential for future biotechnological applications. Many studies also hypothesize that certain traits influence the adaptation and thriving of these bacteria in several marine niches, such as sponges. Of the many recently identified members of the marine clade, only a few have been studied beyond their isolation and initial physiological characterization, while their interactions with sponges have not been investigated at all. In the framework of this study, eight strains previously isolated from two different sponge species (Dysidea avara and Aplysina aerophoba) were identified as member of the Flavobacteriaceae family based on their 16S rRNA gene. Their importance lies in the fact that they are phylogenetically distant from existing members of the marine clade in public databases. To reconstruct their phylogenomic position and investigate their novelty, we will perform whole-genome sequencing and collect publicly available genomes of strains related to all the clades within the Flavobacteriaceae. A comparative genome analysis will follow to discern common traits between the sponge symbionts and elucidate novel biosynthetic pathways affiliated to their secondary metabolism. Ultimate goal is to predict the 'flavobacteria'-sponge interactions and reveal their metabolic machinery based on genomic features.
\end{abstract}




\title{
DEPTH MATTERS: COMPOSITION AND ANTIMICROBIAL ACTIVITY OF MICROBIAL COMMUNITIES ASSOCIATED WITH XESTOSPONGIA MUTA AND AGELAS SP. ALONG A DEPTH GRADIENT
}

\author{
ANAK AGUNG GEDE INDRANINGRAT ${ }^{1,2}$ (ANAK1.INDRANINGRAT@WUR.NL), \\ LEONTINE E. BECKING ${ }^{3,4}$, HAUKE SMIDT ${ }^{1}$, AND DETMER SIPKEMA ${ }^{1}$ \\ ${ }^{1}$ Laboratory of Microbiology, Wageningen University, The Netherlands \\ ${ }^{2}$ Department of Biology, Faculty of Mathematics and Science Education, Institut Keguruan dan \\ Ilmu Pendidikan Persatuan Guru Republik Indonesia (IKIP PGRI) Bali, Indonesia \\ ${ }^{3}$ Marine Animal Ecology, Wageningen University, The Netherlands \\ ${ }^{4}$ Naturalis Biodiversity Center, Leiden, The Netherlands
}

\begin{abstract}
Bacterial communities constitute a significant fraction of a sponge's tissues, and these microorganisms are known to play an important role in producing bioactive compounds to defend their host against predators and/or competitors. Sponge-associated bacteria from shallow water $(\leq 30 \mathrm{~m})$ are the focus of many studies. However, limited information is available from their deeper counterparts. To address this knowledge gap, we sampled 32 sponge individuals of two sponge species: Xestospongia muta and Agelas sp. from three depth ranges $(0-30,30-60,60-90 \mathrm{~m})$ in front of Substation Curaçao in the Dutch Caribbean. Sponge-associated bacteria of these two sponges were cultivated on replicate plates of six culture media. Replicate plates were subsequently used either to pick individual colonies or to scrape the entire biomass. From the colony-picking plates, a total of 3259 and 815 colonies were obtained for Agelas sp. and X. muta, respectively. From these, 181 bacterial colonies comprising 53 different colony morphologies were selected for Agelas sp. and 103 bacterial colonies with 39 different colony morphologies for $X$. muta. Microbial community analysis of these sponge samples, along with identification of the cultivable fraction of sponge-associated bacteria derived from the colony-scraping plates was achieved based on Illumina Miseq sequencing of $16 \mathrm{~S}$ rRNA gene amplicons. Antimicrobial potential of these two sponge species and their isolated bacteria is now being examined by testing crude extracts of each sponge tissue and cell-free supernatant of each isolate against six bacterial and fungal reference strains.
\end{abstract}

Key words: sponges, cultivation, microbial diversity, depth gradient, antimicrobial 


\title{
MOLECULAR ANALYSIS OF MICROBIAL COMMUNITY DYNAMICS DURING IN VITRO FERMENTATION OF FIBRES DERIVED FROM THREE INDONESIAN LOCAL TUBERS BY A HUMAN FECAL INOCULUM
}

\author{
Z.H. HASSAN ${ }^{1,2}$ (ZAHIROTUL.HASSAN@WUR.NL), \\ E.G. ZOETENDAL ${ }^{2}$, H.A. SCHOLS ${ }^{3}$, H. SMIDT ${ }^{2}$ \\ ${ }^{1}$ Indonesian Center for Agricultural Postharvest Research and Development, Indonesian \\ Agency for Agricultural Research and Development, Jl. Tentara Pelajar No. 12 \\ Bogor 16114 West Java, Indonesia \\ ${ }^{2}$ Laboratory of Microbiology, Wageningen University \& Research, Stippeneng 4, \\ 6708 WE Wageningen, The Netherlands \\ ${ }^{3}$ Laboratory of Food Chemistry, Wageningen University \& Research, Bornse Weilanden 9, \\ 6708 WG Wageningen, The Netherlands
}

\begin{abstract}
Each type of fibre, depending on its physicochemical properties, is differently fermented by colonic microbiota, which subsequently leads to different alterations in the microbial community composition. This study aimed to examine the alteration on the microbial composition during in vitro fermentation of three different fibres derived from Indonesian local tubers, including taro (Colocasia esculenta), cassava (Manihot utillisima) and sweet potato (Ipomoea batatas), by human fecal microbiota. The fibres were prepared as alcohol insoluble solids (AIS), and were shown to have unique physico-chemical properties with respect to the starch and protein contents, molecular weight distribution, oligosaccharide profile, constituent monosaccharide composition, degree of acetylation, and degree of methyl esterification. A pool of fecal samples from six healthy Indonesian adults was used as inoculum, and samples were taken throughout the incubation. Fermentation kinetics and metabolite profiles were different for different fibres. DNA was extracted from the fermentation digests using the repeated bead beating method and the microbial community composition was analysed by Illumina HiSeq sequencing of $16 \mathrm{~S}$ ribosomal RNA gene fragments amplified with primer pair of $515 \mathrm{~F}$ (5'-GTGCCAGC[ACG]GCCGCGGTAA) - 806R (5'-GGACTAC[ACT][ACG]GGGT[AT]TCTAAT) targeting the $\mathrm{V} 4$ region. After $6 \mathrm{~h}$ of fermentation, an increase in the relative abundance of Bifidobacterium to $60 \%$ was observed upon cassava fibre fermentation. This number continued to increase to $66 \%$ after $12 \mathrm{~h}$, and slightly decreased to $57 \%$ after $22 \mathrm{~h}$. Dorea was found to dominate $(35 \%)$ the microbial community after $48 \mathrm{~h}$ fermentation. As for sweet potato fibre, a less pronounced increase in the relative abundance of Bifidobacterium to $36 \%$ was observed after $6 \mathrm{~h}$ fermentation, which then decreased to $18 \%$ after $22 \mathrm{~h}$. Next to Bifidobacterium, Dorea, Coprococcus, and Mogibacterium were three genera that were present at a relatively higher abundance at $22 \mathrm{~h}$ and $48 \mathrm{~h}$ upon fermentation of sweet potato fibre, and each constituted over $8 \%$ of the total bacteria. The lowest increase in the relative abundance of Bifidobacterium was observed during taro fibre fermentation, i.e. to $30 \%$ after $6 \mathrm{~h}$ and $12 \mathrm{~h}$. After $22 \mathrm{~h}$ upon taro fibre fermentation, Coprococcus dominated the microbial community, reaching a relative abundance of almost $50 \%$ at $48 \mathrm{~h}$ after fermentation. In conclusion, the alterations in microbial community composition were distinctive for each fibre as a result of their unique properties.
\end{abstract}

Keywords: in vitro fermentation, microbial composition, fibre, local Indonesian tubers. 


\title{
ACETATE AS METABOLIC TARGET IN THE PREVENTION OF OVERWEIGHT-RELATED CHRONIC METABOLIC DISORDERS
}

\author{
GERBEN D.A. HERMES ${ }^{1}$ (GERBEN.HERMES@WUR.NL), \\ EMANUEL E. CANFORA ${ }^{2}$, KOEN VENEMA ${ }^{3}$, ERWIN G. ZOETENDAL ${ }^{1}$, ELLEN E. BLAAK ${ }^{2}$ \\ ${ }^{1}$ Labaratory of Microbiology, Wageningen University \& Research, The Netherlands \\ ${ }^{2}$ Department of Human Biology, NUTRIM School of Nutrition and Translational Research in \\ Metabolism, Maastricht University Medical Centre + , The Netherlands \\ ${ }^{3}$ Department of Human Biology, NUTRIM School of Nutrition and Translational Research in \\ Metabolism, Maastricht University Medical Centre +, Campus Venlo, The Netherlands
}

\begin{abstract}
The gut microbiota has been suggested to be highly involved in the etiology of obesity and type 2 diabetes mellitus. Gut-derived short chain fatty acids (SCFA), formed by microbial fermentation of indigestible carbohydrates, might be involved in the control of body weight and insulin sensitivity. Recently, it was shown that acute infusions of acetate and SCFA mixtures in the amount achieved by a high fibre diet, in the distal, but not in the proximal part, of the colon had pronounced effects on lipolysis, fat oxidation and inflammatory profile. This project will further explore the effects of acetate levels in the distal colon and systemic circulation in relation to effects on human metabolism and insulin sensitivity using in-vitro and in-vivo approaches. Using TIM-2, a validated model of the colon, single dietary fibres and combinations with resistant starch will be screened for fermentation time and acetate production using a microbiota from obese, prediabetic and 'metabolically healthy' subjects, to identify key microbes and pathways that are responsible for the biosynthesis of high distal acetate production. The obtained results will be applied to design a human dietary intervention study, aimed at investigating the effect of long-term fibre supplementation in reversing high fat-induced insulin resistance to provide information for new strategies in nutritional prevention of insulin resistance and chronic metabolic diseases in adults and elderly.
\end{abstract}




\title{
SCREENING ALGAL-BACTERIAL INTERACTIONS FOR NEW MOLECULES
}

\author{
JIE LIAN ${ }^{1}$ (JIE.LIAN@WUR.NL), \\ RENE H. WIJFFELS ${ }^{2,3}$, HAUKE SMIDT ${ }^{1}$, DETMER SIPKEMA ${ }^{1}$ \\ ${ }^{1}$ Laboratory of Microbiology, Wageningen University \& Research, Wageningen, \\ The Netherlands \\ ${ }^{2}$ Bioprocess Engineering, AlgaePARC, Wageningen University \& Research, Wageningen, \\ The Netherlands \\ ${ }^{3}$ Faculty of Biosciences and Aquaculture, N8049, Bodø, Norway
}

A majority of microbial biosynthetic gene clusters only stay active when microbes are interacting with each other using secondary metabolites. Investigating "microbial communications" and small molecules that mediate them provide an appealing research strategy for screening such molecules with potential applications. Widespread and naturaloccurring algal-bacterial symbiosis provides an ideal model for such research. So we isolated bacteria from algal cultivation reactors, and have been testing their influences individually on sterile algal host in 24-well microplate. When growing with algal host, bacteria might switch on some biosynthetic genes that are normally inactive under typical laboratory conditions. These silent genes represent a huge and hidden reservoir for new molecules. HPLC and mass chromatography will be used to identify and characterize these unknown molecules. Derived novel molecules might be able to affect algal physiology and modulate algal populations, which is of particular importance in algal cultivation. Furthermore, there has evidence that some compounds turn out to be antimicrobial compounds that can kill algal hosts or other bacteria, which can be exploited for aquaculture and human medical practice. Therefore, we are exploiting algal-bacterial interactions for those cryptic metabolic chemicals. 


\title{
VITAMIN $B_{12}$ MEDIATED DECHLORINATION IN PRISTINE
} HYPERSALINE LAKES

\author{
PENG PENG ${ }^{1 \#}$ (PENG.PENG@WUR.NL), \\ YUE LU ${ }^{1,6,7 \#}$, ALEXANDER RUECKER ${ }^{2,3}$, JAVIER RAMIRO-GARCIA ${ }^{1,4}$, ALEXANDER UMANETS ${ }^{1}$, \\ ANDREAS KAPPLER ${ }^{3,5}$, DETMER SIPKEMA ${ }^{1}$, SIAVASH ATASHGAHI ${ }^{1}$, HAUKE SMIDT $^{1}$ \\ ${ }^{1}$ Laboratory of Microbiology, Wageningen University \& Research, The Netherlands \\ ${ }^{2}$ Biogeochemistry-Baruch Institute of Coastal Ecology and Forest Science, \\ Clemson University, USA \\ ${ }^{3}$ Geomicrobiology, Center for Applied Geosciences, University of Tuebingen, Germany \\ ${ }^{4}$ Laboratory of Systems and Synthetic Biology, Wageningen University \& Research, \\ The Netherlands \\ ${ }^{5}$ Center for Geomicrobiology, Department of Bioscience, Aarhus University, Denmark \\ ${ }^{6}$ College of Environmental Science and Engineering, Hunan University, China \\ ${ }^{7}$ Key Laboratory of Environmental Biology and Pollution Control, Hunan University, \\ Ministry of Education, China
}

Biotic formation of chloromethane and chloroform was reported from the hypersaline Lake Strawbridge in Australia. Assuming that natural occurrence of organohalides can prime development of corresponding dehalogenating microbes, we prepared anoxic microcosms from Lake Strawbridge sediments. Dechlorination of amended chloroform and tetrachloroethene (PCE) to dichloromethane and trichloroethene was observed, respectively. The chloroform dechlorination rates decreased in the subsequent sediment-free transfers compared to the original enrichment cultures. However, increasing the vitamin $B_{12}$ concentration from 0.03 to $4 \mu \mathrm{M}$ significantly enhanced chloroform dechlorination rates. Archaeal 16S rRNA gene copy numbers declined more than four orders of magnitude in transfer cultures, excluding their role in chloroform dechlorination. Known organohalide respiring bacteria (OHRB) for dechlorination of chloroform and/or PCE were neither detected in sediment microcosms nor transfer cultures using MiSeq bacterial 16S rRNA gene analysis and quantitative PCR, suggesting the presence of bacteria capable of co-metabolic dechlorination of chloroform mediated by vitamin $\mathrm{B}_{12}$ or yet unknown OHRB. Combined with previously documented biotic organohalogen formation, this suggests potential interdependencies between microbial chlorinators and dechlorinators in local chlorine cycles at hypersaline lakes.

\# equal contribution 


\title{
THE EFFECTS OF SYNBIOTIC SUPPLEMENTATION ON SMALL INTESTINE AND FECAL MICROBIOTA
}

\author{
RAN AN ${ }^{1}$ (RAN.AN@WUR.NL), \\ ELLEN WILMS ${ }^{2}$, COLINE GERRITSEN ${ }^{1,3}$, CELIA SEGUI PEREZ ${ }^{1}$, GER T. RIJKERS ${ }^{4,5}$, \\ AD A.M. MASCLEE ${ }^{2}$, FRED J. TROOST ${ }^{2}$, ISOLDE BESSELING-VAN DER VAART ${ }^{3}$,
} ERWIN G. ZOETENDAL ${ }^{1}$, HAUKE SMIDT ${ }^{1}$

\author{
${ }^{1}$ Laboratory of Microbiology, Wageningen University, Wageningen, The Netherlands \\ ${ }^{2}$ Division Gastroenterology-Hepatology, NUTRIM School of Nutrition and Translational Research \\ in Metabolism, Maastricht University Medical Center+, Maastricht, The Netherlands \\ ${ }^{3}$ Winclove B.V., Amsterdam, The Netherlands \\ ${ }^{4}$ Science Department, University College Roosevelt, Middelburg, the Netherlands \\ ${ }^{5}$ Department of Medical Microbiology and Immunology, St. Antonius Hospital, Nieuwegein, \\ The Netherlands
}

The human gastrointestinal tract harbors a large number of microbes, called the microbiota, which plays an important role in our health. Although different microbial communities exist along the gastrointestinal tract, fecal samples are exclusively used to study the impact of preand probiotics on the microbiota. In this project, we studied the effect of the multispecies probiotic Ecologic $\circledR 825$ in combination with prebiotic short chain fructo-oligosaccharides (FOS P6) on fecal microbiota and the microbial communities inhabiting different parts of the small intestine.

A randomized double-blind placebo-controlled parallel design was performed in this study. 20 healthy volunteers, with no gastrointestinal complaints history were recruited. The volunteers were supplemented with either synbiotic or placebo twice daily for 14 days. Before and after the supplementation period, samples from different locations of the small intestine were collected using a multi-lumen customized sampling catheter in addition to fecal material. For microbiota profiling, DNA was isolated from the samples followed by amplification of the variable regions V1-V2 of the 16S rRNA gene, sample-specific barcoding and subsequent MiSeq illumine sequencing.

At baseline, no major differences in bacterial composition were found between the microbiota of duodenum, ileum and jejunum. However, they were significantly different from the fecal microbiota, in terms of the compositions and diversities. Surprisingly, the small intestinal microbiota was more diverse than fecal microbiota $(P<0.05)$. Although a trend for higher relative abundance of Bifidobacterium, Lactobacillus and Lactococcus was found after synbiotic supplementation compared to placebo, no significant synbiotic effect was observed $(P>0.05)$, in line with the previously observed lack of significant effects on intestinal permeability, immune function or gastrointestinal symptoms in subjects receiving the synbiotic supplement.

In conclusion, two weeks supplementation of Ecologic ${ }^{\circledR} 825$ combined with FOS P6 did not show a significant synbiotic effect on the microbiota composition of the small and large intestine. 


\title{
SCREENING FOR ANTIMICROBIAL ACTIVITY OF MARINE ACTINOBACTERIA FROM LEMBEH STRAIT, INDONESIA
}

\author{
SHANTI RATNAKOMALA ${ }^{1}$, AKHIRTA ATIKANA ${ }^{1,2}$ (AKHIRTA.ATIKANA@WUR.NL), \\ IKA NURZIJAH ${ }^{1}$, MIRANTI NURINDAH SARI ${ }^{1}$, FAHRURROZI $^{1}$, \\ PUSPITA LISDIYANTI ${ }^{1}$, HAUKE SMIDT ${ }^{2}$, DETMER SIPKEMA ${ }^{2}$ \\ ${ }^{1}$ Laboratory of Applied Microbiology, Research Center for Biotechnology, \\ Indonesian Institute of Sciences (LIPI), Indonesia \\ ${ }^{2}$ Laboratory of Microbiology, Wageningen University \& Research, \\ Wageningen, The Netherlands
}

\begin{abstract}
Actinobacteria are Gram-positive bacteria and have been widely explored as a robust producer of the antibiotic compounds. Indonesia has a high diversity of Actinobacteria. In this study, we isolated the actinobacteria from the marine samples (seawater, sea-sediment, and marine sponges) collected from Lembeh Strait, North Sulawesi to explore their antibacterial activity. A total of 50 actinobacteria isolates were tested against six indicator microorganisms: Bacillus subtilis BTCC B-612, Escherichia coli BTCC B-609, and Stapylococcus aureus BTCC B-611, Saccharomyces cerevisae BTCC Y-34, Candida albicans BTCC Y-33, and Micrococcus luteus BTCC B-552, and 24 isolates showed antimicrobial activity. Of these 24 isolates, 10 isolates were selected for ethyl acetate extraction. The ethyl acetate extracts were tested against B. subtilis, E. coli, S. aureus and M. luteus in 96 wells plates using the resazurin assay. Three ethyl acetate extracts from actinobacterial isolates designated as BLH 5-22, BLH 3-12 and BLH 1-22 showed the highest antibacterial activity. Further identification using 16S ribosomal RNA gene sequence showed that the BLH 1-22 isolate is closely related to Micromonospora sp. $(99 \%)$ that has been proven as an antibiotic producer. This result further highlights the antibacterial potential of Indonesian marine actinobacteria.
\end{abstract}

Keywords: marine actinobacteria, Lembeh, antibacterial activity 


\title{
THE EFFECT OF FIBER AND PREBIOTICS ON CHILDREN'S GASTROINTESTINAL DISORDERS AND MICROBIOME
}

\author{
CARRIE A.M. WEGH ${ }^{1,2}$ (CARRIE.WEGH@WUR.NL), MARGRIET H.C. SCHOTERMAN ${ }^{3}$, \\ ELAINE E. VAUGHAN ${ }^{4}$, CLARA BELZER $^{1 *}$ MARC A. BENNINGA ${ }^{2 *}$ \\ ${ }^{1}$ Laboratory of Microbiology, Wageningen University and Research, Wageningen, \\ The Netherlands \\ ${ }^{2}$ Department of Pediatric Gastroenterology and Nutrition, Emma's Children's Hospital Academic \\ Medical Center, Amsterdam, The Netherlands \\ ${ }^{3}$ FrieslandCampina, Amersfoort, The Netherlands \\ ${ }^{4}$ Sensus BV (Royal Consun), Roosendaal, The Netherlands \\ * these authors contributed equally
}

Introduction: Bacteria received upon birth are the start of colonization of $10^{14}$ bacteria that are present in the mature human gastrointestinal tract, better known as the microbiota. The gut microbiota are implicated in gastrointestinal health, nutrient metabolism and benefits such as prevention of infection. Dietary fiber, including prebiotics, escape digestion in the small intestine and reach the colon intact, where they are partially or completely fermented by the gut microbiota.

Areas covered: The possible interactions between dietary fiber, prebiotics and microbiota are discussed as well as how this relates to functional gastrointestinal disorders. During the first years of life the microbiome has not yet reached a stable state and is sensitive to disturbance by environmental factors. An imbalance in the microbiota early in life is found to be associated with several functional gastrointestinal disorders such as colic, functional abdominal pain, irritable bowel syndrome and constipation.

Expert commentary: Better understanding of how microbial changes in early-life can impact health might lead to new treatments or target disease prevention. Nutritional strategies with fibers or prebiotics at an early age might be useful as they are able to change colonic microbiota composition and its metabolic activity e.g. growth stimulation of Bifidobacterium and Lactobacillus. 


\title{
THE POTENTIAL OF PREBIOTIC EFFECT ON MICROBIOTA COLONIZATION, GUT FUNCTION AND ANIMAL PERFORMANCE
}

\author{
CAIFANG WEN (CAIFANG.WEN@WUR.NL), \\ PROF. HAUKE SMIDT (WUR-MICROBIOLOGY) \& PROF. H.A. (HENK) SCHOLS
}

Wageningen University \& Research, Food Chemistry

\begin{abstract}
Introduction
In the past decades, studies have widely confirmed that both bacterial and fungal microorganisms within the intestinal microbiota play a vital role in the development and function of the gastrointestinal tract and in gut health. In the veterinary field, efforts are made to reduce antibiotic use in swine production, thus increasing diet complexity is, without doubt, a sustainable option to increase microbial diversity and the abundance of beneficial microbes to help reduce the incidence diarrhea and decrease sub-therapeutic antibiotic use. To this end, there is growing interest in the use of prebiotics as alternatives to antibiotic use for animal feeding. In recent years, different new prebiotics have been placed on the market. New prebiotics will be studied with respect to their effect on animal performance.
\end{abstract}

Aim

This project aims to investigate the potential of new prebiotics on microbiota (bacteria and fungi) colonization, gut function and animal performance.

\section{Approach}

The objective of this project is to study the effects of prebiotic feed materials on gut composition and function in broilers and pigs.

The physico-chemical properties of the prebiotics will be characterized. In vitro fermentation, in vitro cell based assays and in vitro adhesion assays will be used to assess potential prebiotic and colonization resistance-enhancing effects. Furthermore, broilers and pigs will be used to investigate the effects of a diet intervention with this prebiotics on gut microbiota composition (bacteria and fungi) and functioning using 16S rRNA and ITS2 region profiling, metatranscriptomics and metabolome analysis. 


\title{
RELEASE, DEGRADATION AND DETECTION OF PHENYLPROPIONATE DURING ANAEROBIC DEGRADATION OF RICE STRAW
}

\author{
Y. ZHENG ${ }^{1}$ (YING1.ZHENG@WUR.NL), \\ P. WORM ${ }^{1}$, T.K.S. JANSSENS ${ }^{2}$, A.H. VAN GELDER ${ }^{1}$, T.E. DE BOER ${ }^{2}$, A. BROUWER ${ }^{2}$, \\ A.J.M. STAMS ${ }^{1}$, H. SMIDT ${ }^{1}$, S. ATASHGAHI ${ }^{1}$ \\ ${ }^{1}$ Wageningen University \& Research, Wageningen, The Netherlands \\ 2 MicroLife Solutions B. V., Amsterdam, The Netherlands
}

Rice straw, the world's third largest agricultural residue, is widely used for biofuel production. Anaerobic digestion of rice straw to biogas represents an environmentally friendly and costeffective treatment. However, the complex lignocellulose structure of rice straw stabilized/crosslinked by mono-aromatic compounds such as ferulate makes it difficult to degrade without pretreatment ${ }^{1}$. 3-phenylpropionate (PPA) is a key intermediate of anaerobic degradation of ferulate ${ }^{2}$ and rice straw ${ }^{3}$. Therefore, PPA production can indicate opening of the lignocellulose structure in rice straw and hence increased access of degrading microbes to carbohydrates. This study aimed to investigate the source and fate of PPA during anaerobic degradation of rice straw and ferulate and the microbes involved, and to develop a fast PPA detection method that can be used to monitor anaerobic digestion progress. Stable methanogenic rice straw-degrading enrichment cultures were obtained using swine manure digester sludge as an inoculum. Successive enrichments using either ferulate or PPA as the sole carbon source showed rapid anaerobic degradation. PPA degradation as the sole carbon source led to transient acetate and hydrogen production, whereas benzoate and methane were detected as the end products. Similar end products were found in rice straw- and ferulatedegrading enrichments with PPA as a degradation intermediate. Microbial community analysis by MiSeq 16S rRNA gene sequencing revealed Clostridiales and Bacteroidales as the dominant bacterial taxa in rice straw-degrading enrichments. The results of ongoing bacterial and archaeal community analyses from ferulate- and PPA-degrading enrichments and metagenomic analysis from PPA-degrading enrichment will be presented. Lastly, a luminescence pHcARLux assay was developed for rapid detection of PPA as the key intermediate of rice straw and ferulate degradation. This newly developed reporter construct has the potential to quickly indicate ferulate degradation and thereby monitor the opening of the lignocellulose structure during rice straw degradation.

1. Grbić-Galić D, Young L Y. Methane fermentation of ferulate and benzoate: anaerobic degradation pathways. Applied and Environmental Microbiology, 1985, 50(2): 292-297.

2. Marcia $M O$. Feruloylation in grasses: current and future perspectives. Molecular Plant, 2009, 2(5): 861-872.

3. Glissmann K, Hammer E, Conrad R. Production of aromatic compounds during methanogenic degradation of straw in rice field soil. FEMS Microbiology Ecology, 2005, 52(1): 43-48. 


\title{
ASSOCIATION BETWEEN DURATION OF INTRAVENOUS ANTIBIOTIC ADMINISTRATION AND EARLY LIFE MICROBIOTA DEVELOPMENT IN LATE PRETERM INFANTS
}

\author{
R.D. ZWITTINK ${ }^{1}$; I.B. RENES ${ }^{2}$; R.A. VAN LINGEN ${ }^{3}$; D. VAN ZOEREN-GROBBEN ${ }^{3}$; \\ P. KONSTANTI ${ }^{1}$; O.F. NORBRUIS ${ }^{3}$, R. MARTIN ${ }^{2}$; L.J.M. GROOT JEBBINK ${ }^{3}$; \\ J. $\mathrm{KNOL}^{1,2, \mathrm{x}} ; \mathrm{C}$. BELZER ${ }^{1, \mathrm{x}}$ \\ ${ }^{1}$ Laboratory of Microbiology, Wageningen University, Wageningen, The Netherlands \\ ${ }^{2}$ Nutricia Research, Utrecht, The Netherlands \\ ${ }^{3}$ Princess Amalia Children's Centre, Dept. of Paediatrics and Neonatology, Isala, Zwolle, \\ The Netherlands \\ ${ }^{x}$ These authors contributed equally
}

\begin{abstract}
Antibiotic treatment is common practice in the neonatal ward for prevention and treatment of sepsis, which is one of the leading causes of mortality and morbidity in preterm infants. Applied antibiotic strategies in neonatology led to decreased mortality and morbidity rates, however, there is a risk of impeding gut microbiota development. Although the effect of antibiotic treatment on microbiota development has been well recognised, not much attention has been paid to duration of treatment. Our aim is to study the effect of antibiotic treatment duration on intestinal microbiota development in preterm infants. Faecal samples from fifteen preterm infants (35 \pm 1 weeks gestation; $2871 \pm 260$ gram birthweight) were collected during the first six postnatal weeks, including a sample before and after antibiotic treatment. The infants were stratified according to antibiotic treatment duration; no, short ( $<3$ days) or long ( $>5$ days) treatment with amoxicillin/ceftazidime during the first postnatal week. Microbiota composition was determined by sequencing of the 16S rRNA gene and qPCR. Preterm infants faecal microbiota was dominated by Bifidobacterium throughout the first six postnatal weeks. Short and long antibiotic treatment during the first postnatal week drastically disturbed normal colonisation pattern. Antibiotic treatment was effective against members of the Enterobacteriaceae family, but also negatively affected Bifidobacterium abundance and allowed Enterococcus to thrive. Enterococcus remained dominant for up to two weeks after antibiotic treatment discontinuation. Short, but not long, antibiotic treatment allowed for recovery of Bifidobacterium levels within the first six postnatal weeks. Overall, our findings show that antibiotic administration during the first postnatal week greatly affects infant gastrointestinal microbiota composition, however, quick cessation of antibiotic treatment allows for recovery of the microbiota. Disturbances in microbiota development caused by short and more extensively by long antibiotic treatment, could affect healthy development of the infant via interference with maturation of the immune system and gastrointestinal tract.
\end{abstract}




\section{Poster presentations Microbial physiology \\ Presented in alfabetical order}




\title{
SULFATE-REDUCING CONSORTIA CONSUMING SUBSTRATE AT ACIDIC CONDITIONS
}

\author{
NOHEMI CAMPOS-QUEVEDO ${ }^{1}$ (NOHEMI.CAMPOSQUEVEDO@WUR.NL), \\ ELIAS RAZO-FLORES ${ }^{1}$, NGUYEN E. LOPEZ-LOZANO ${ }^{1}$, IRENE SÁNCHEZ-ANDREA ${ }^{2}$, \\ LOURDES B. CELIS ${ }^{1}$ \\ ${ }^{1}$ Instituto Potosino de Investigación Científica y Tecnológica. San Luis Potosí, SLP. México \\ ${ }^{2}$ Wageningen University, Wageningen, The Netherlands
}

Acidic effluents from mining and metallurgical industries usually have high concentrations of sulfate $(\mathrm{g} / \mathrm{L})$, and dissolved metals (e. gr. Fe, $\mathrm{Ni}, \mathrm{Pb}, \mathrm{As}$ ), and it causes several environmental problems. Biological treatment based on sulfate reducing bacteria (SRB) is often a viable option due to lower costs and the formation of more stable precipitates, compared to physicochemical processes. SRB produce sulfide, which reacts with dissolved metals, forming precipitates (metal sulfides). However, the major drawback is that acetate cannot be degraded

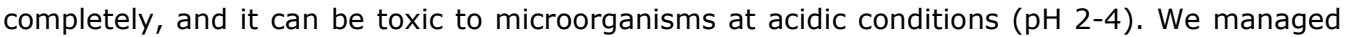
to obtain 7 different cultivable consortia by traditional microbiology techniques (successive transfers), from a natural acidic environment ( $\mathrm{pH}$ around 2 ). These consortia completely consume the substrate at acidic $\mathrm{pH}$, either lactate or glycerol. Using molecular techniques (cloning-sequencing) it was possible to obtain the composition of the 7 cultivable consortia, which showed to have a great heterogeneity, although they were obtained from the same sediment, reflecting the effect of the culture conditions on the community. At phylum level was found Bacteroidetes (10-65\%), Firmicutes (10-55), Proteobacteria (5-45\%), Spirochaetae $(10-40 \%)$, Synergestes $(10-20 \%)$ and sequences that were not related or no described yet $(5-10 \%)$ in all of the consortia. Closest relative to Desulfatirhabdium butyrativorans was almost present in all the consortia with $2-13 \%$ of the sequences belonged to it, with a $96-98 \%$ of similarity. Efforts were made to try to isolate this microorganism of interest that may be the responsible of the complete consumption of substrate at acidic $\mathrm{pH}$, by serial dilution and solid plate experiments. Production of sulfide was measured (5days), and DAPI technique with a specific probe for SRB, with this we were able to identify the morphology of the microorganism of interest. Serial dilutions still made until we have the isolate of the microorganism of interest. 


\title{
GROW OR STORE? EXPLORING METABOLIC DECISION MAKING UNDER FEAST/FAMINE CONDITIONS USING DYNAMIC 13C FLUX ANALYSIS
}

\author{
LEONOR GUEDES DA SILVA ${ }^{1 \S}$ (L.GUEDESDASILVA@TUDELFT.NL), \\ KOEN VERHAGEN ${ }^{1}$, ANDY WIRANATA WIJAYA ${ }^{1}$, ROBBERT KLEEREBEZEM ${ }^{1}$, \\ MARK C.M. VAN LOOSDRECHT ${ }^{1}$, ALJOSCHA S. WAHL $^{1}$ \\ ${ }^{1}$ Department of Biotechnology, Delft University of Technology, Delft, The Netherlands \\ $\S$ equal contribution
}

\begin{abstract}
Natural habitats of microorganisms are dynamic environments with non-continuous supply of carbon and energy sources, in which intermediate storage of substrates can increase competitiveness. Plasticicumulans acidivorans are polyhydroxybutyrate (PHB) accumulating bacteria enriched from activated sludge using carbon feast-famine cycles as selective pressure. Despite growing slowly, P. acidivorans outcompetes other bacteria by quickly taking up acetate and storing it intracellularly as PHB to later use it for growth. As soon as acetate is depleted, these bacteria immediately 'switch' their metabolism from PHB production to consumption entailing a very interesting regulatory challenge as parallel activity could lead to significant losses (futile cycling). While the stoichiometry for both feast and famine phases has been extensively described in literature, the switch regulation is not yet fully understood.
\end{abstract}

To elucidate the responsible regulatory processes, an enrichment of P. acidivorans was studied using targeted intracellular metabolite analysis over time, with emphasis on the feast to famine switch. In combination with extracellular rates, the measured intracellular metabolite pools are used to design a labelling experiment to obtain actual intracellular fluxes (dynamic $13 \mathrm{C}$ flux analysis). Here the challenge is to create an isotopically non-stationary state (usually mediated by changing the substrate's isotopic composition) to study the metabolic response in the transition from presence-to-absence of substrate.

In this way, we aim to unravel the responsible regulatory mechanism governing the metabolic switch from storage-to-consumption and use this knowledge not only to understand its ecological relevance, but to also propose novel metabolic strategies for microbial cell factory design. 


\title{
ACIDOTHERMOPHILIC BIOREDUCTION OF SULFUR FOR THE RECOVERY OF VALUABLE METALS
}

\author{
C.M. VAN DER GRAAF (LOT.VANDERGRAAF@WUR.NL), \\ A.J.M. STAMS, I. SÁNCHEZ-ANDREA
}

Wageningen University, Department of Microbiology, The Netherlands

\begin{abstract}
Base metals and rare earth elements are important raw materials for a wide range of technological/industrial applications. A shortage of these materials is foreseen in the future, as their production cannot keep up with demand. The hot, acidic, metal-contaminated waste streams from mining operations are a potential additional source of these metals, which can be recovered from solution as metalsulfide precipitates by supplying hydrogen sulfide $\left(\mathrm{H}_{2} \mathrm{~S}\right)$. Microbial synthesis of $\mathrm{H}_{2} \mathrm{~S}$ is of great interest, as it has important sustainability advantages over physicochemical methods such as addition of calcium carbonate $\left(\mathrm{CaCO}_{3}\right) . \mathrm{H}_{2} \mathrm{~S}$ is the most reduced form of sulfur, and microbial $\mathrm{H}_{2} \mathrm{~S}$ synthesis is a result of the reduction of the more oxidized sulfur compounds sulfate $\left(\mathrm{SO}_{4}{ }^{2-}\right)$, sulfite $\left(\mathrm{SO}_{3}{ }^{2-}\right)$, thiosulfate $\left(\mathrm{S}_{2} \mathrm{O}_{3}{ }^{2-}\right)$ and elemental sulfur (commonly denoted as $\mathrm{S}_{8}{ }^{0}$ ). This project focuses on the development of mixed cultures and the isolation of specific strains that reduce elemental sulfur to $\mathrm{H}_{2} \mathrm{~S}$ under acidothermophilic conditions, comparable to the temperature and acidity of mining waste streams. Enrichment experiments will be carried out at a range of $\mathrm{pH}(<4)$ and temperatures $\left(>70^{\circ} \mathrm{C}\right)$, with organic and inorganic electron donors, using biomass samples obtained from natural environments characterized by high $\mathrm{T}$ and low $\mathrm{pH}$ as inocula (e.g.: volcanic hot springs in Yellowstone National Park). Cultures producing $\mathrm{H}_{2} \mathrm{~S}$ will be selected for further enrichment, and cultivation will be scaled up to a $10 \mathrm{~L}$ bioreactor. Simultaneously, specific strains will be isolated to (i) study $\mathrm{H}_{2} \mathrm{~S}$ synthesis in pure and defined mixed culture, and (ii) study the physiology of acidothermophilic sulfur reduction in detail. Of specific interest are (i) the chemical form in which elemental sulfur is used as electron acceptor, as elemental sulfur has low solubility in water, (ii) the mechanisms used for energy conservation at low extracellular $\mathrm{pH}$, and (iii) the stress tolerance mechanisms used to cope with acidothermophilic conditions.
\end{abstract}




\title{
PROPIONIBACTERIUM SP. STRAIN JV5: A NOVEL PROPIONATE PRODUCER ISOLATED FROM RUMINAL FIBROUS CONTENT OF A DAIRY COW
}

\author{
JUEELI D. VAIDYA ${ }^{1,2}$ (JUEELI.VAIDYA@WUR.NL), \\ BASTIAN HORNUNG ${ }^{3}$, JOAN E. EDWARDS ${ }^{1,2}$, HAUKE SMIDT ${ }^{2}$ AND CAROLINE M. PLUGGE ${ }^{2}$ \\ ${ }^{1}$ Top Institute Food and Nutrition, Wageningen, The Netherlands \\ ${ }^{2}$ Laboratory of Microbiology, Wageningen University \& Research, Wageningen, \\ The Netherlands \\ ${ }^{3}$ Laboratory of Systems and Synthetic Biology, Wageningen University \& Research, \\ Wageningen, The Netherlands
}

\begin{abstract}
A novel Propionibacterium, strain JV5, was isolated from the ruminal fibrous content of a Holstein Friesian dairy cow. The strain tested Gram-positive, is non-motile, non-sporulating, oxygen tolerant and grew on cellulose agar plates as circular opaque white colonies. The $16 \mathrm{~S}$ ribosomal RNA gene sequence of strain JV5 revealed that it belongs to the genus Propionibacterium with $98 \%$ and $97 \%$ sequence identity to $P$. australiense and $P$. acidifaciens, respectively. Strain JV5 was able to ferment arabinose, adonitol, glucose, lactose, mannose, ribose, saccharose, salicin, sorbitol and trehalose to mainly propionate and acetate, and formate in some cases. Strain JV5 was urease, catalase and indole negative. Its $\mathrm{pH}$ range for growth was $6.5-8$, with an optimum at $\mathrm{pH} 7$. Strain JV5 can grow at temperatures between $35^{\circ} \mathrm{C}$ and $45^{\circ} \mathrm{C}$, with an optimum at $39^{\circ} \mathrm{C}$. Preliminary results showed that cells of strain JV5 attach to the surface of grass silage fibres, suggesting a role in fibre degradation. Additionally, JV5 was able to utilize representative plant-derived polysaccharides including xylan (from both birchwood and oatspelt) and pectin, further supporting its role in fibre degradation. The G + C \% of strain JV5 was $66 \%$. Further genotypic comparison of strain JV5 to the type strain of $P$. australiense (DSM 15818 based on its draft genome sequence (gANI of $88.3 \%$ ) and experimental DNA-DNA hybridization $(65.5 \%)$ placed JV5 at the species border to $P$. australiense. Chemotaxonomic analysis of strain JV5 revealed the presence of meso - diamino pimelic acid (DAP) isomers which were different from P.acidifaciens but similar to $P$.australiense. There was a lack of repiratory quinones in JV5 and the closest relatives. The cellular fatty acids detected were different when compared to the closest relatives of JV5. Based on these phylogenetic, chemotaxonomic and physiological characteristics strain JV5 represents a novel species belonging the genus Propionibacterium.
\end{abstract}




\section{Poster presentations Bacterial genetics}

Presented in alfabetical order 


\title{
MODEL-DRIVEN DESIGN OF NOVEL PSEUDOMONAS PUTIDA STRAINS
}

\author{
C. BATIANIS (CHRISTOS.BATIANIS@WUR.NL), \\ R. VAN HECK, S. DAMALAS, R. VOLKERS, V. DOS SANTOS
}

Laboratory of Systems and Synthetic Biology, Wageningen University, Wageningen, $6703 \mathrm{HB}$, The Netherlands

\begin{abstract}
Microbial biotechnology aims to exploit the huge microbial diversity by integrating microbial systems in industry, environment and medicine. Pseudomonas putida KT2440 is the beststudied pollutant degrading bacterium, that also has a high potential as an efficient production platform of bulk- and fine-chemicals. Ideally, this potential could be expanded by using genome-scale metabolic models (GSMMs) that theoretically enable the design of production strains. Three GSSMs for $P$. putida KT2440 have been published so far but the room for improvement seems to be large. Here, we utilized the $P$. putida KT2440 GSSM iJP962 in order to design a mutant able to overproduce pyruvate. Guided by the strain optimization algorithm RobustKnock, we successfully deleted the gene gnd and the operon aceEF resulting in the proposed genotype, $P$. putida KT2440 $\triangle$ gnd- $\triangle a c e E F$. This double deletion significantly increased the pyruvate production, demonstrating in this way the predictive power of the model-driven strain design strategies. The standardization of such strategies is something that is currently missing, therefore, we propose a model-driven pipeline which can be used for designing novel $P$. putida strains.
\end{abstract}




\title{
THE METABRICK PLATFORM FOR DNA MANIPULATION AND STANDARDIZATION. BRIDGING SYNTHETIC BIOLOGY STANDARDS FOR OPTIMIZED INTEROPERABILITY
}

\author{
STAMATIOS DAMALAS
}

\begin{abstract}
The MetaBrick platform is a toolbox consisted of the MetaBrick assembly method along with the use of engineered MetaBrick parts. It was designed to simplify and optimize plasmid and DNA device construction for synthetic biology applications. Since the infancy of Synthetic Biology there have been several standards and methods for the construction of libraries of vectors and parts. This plethora has ultimately led to standards incompatibility making parts and methodologies not interoperable with each other. The MetaBrick platform is proposed as the solution to the situation. The MetaBrick assembly is the proposed cloning method while in terms of parts standardization we adopted the engineered SEVA platform for vector backbone construction and along with it the use of BioBrick compatible parts for construction of vector inserts. The MetaBrick assembly is a directional and scar-less type-IIs assembly which uses PCR amplified compatible parts. In comparison to the similar GoldenGate, it doesn't need complicated exchange and directional vectors since directionality is applied through the engineered primers during PCR amplification. In comparison to the Gibson assembly, another scar-less method based on PCR amplification, it uses shorter overhangs allowing construction of standardized libraries. The MetaBrick assembly along with primer design was used to optimize the BioBrick assembly by eliminating the need for different restriction enzymes and multiple cloning steps while allowing multipart assemblies. Primer engineering was applied for the construction of parts including, RBS carrying proteins, fusions, tagged proteins and standardized parts from E. coli genes. To increase the versatility of the platform, a vector was engineered based on the SEVA skeleton, utilizing the SEVA modules as Bricks from a newly constructed repository of parts. To make the MetaBrick vector more modular and BioBrick compatible, the SEVA cloning site was replaced by the MetaBrick cloning site (MCS 2.0), compatible with the MetaBrick assembly for BioBricks.
\end{abstract}




\title{
MODIFICATIONS OF THE PYRUVATE DEHYDROGENASE COMPLEX OF E. COLI AND THEIR EFFECT ON ENZYME ACTIVITY AND GROWTH
}

\author{
P.L. FOLCH ${ }^{1}$ (PAULINE.FOLCH@WUR.NL), \\ G. EGGINK ${ }^{1,2}$, J.P.M. SANDERS ${ }^{2}$, R.A. WEUSTHUIS ${ }^{1}$
}

${ }^{1}$ Bioprocess Engineering, Wageningen University \& Research, The Netherlands

${ }^{2}$ Food \& Biobased Research, Wageningen University \& Research, The Netherlands

\begin{abstract}
The pyruvate dehydrogenase complex is a key enzyme of the central metabolism of $E$. coli. It catalyzes the conversion of pyruvate into acetyl-CoA and $\mathrm{CO}_{2}$ using $\mathrm{NAD}^{+}$as cofactor. Acetyl$\mathrm{COA}$ and the reduced cofactor can be used for the production of a multitude of chemicals, preferably under anaerobic conditions. The PDH complex however, is inhibited by the high NADH concentrations that occur when oxygen is absent. For synthesis of some products NADPH is the favored cofactor. Changing both sensitivity to NADH and cofactor specificity is therefore of interest.
\end{abstract}

Previous research has shown that the NADH inhibition and cofactor specificity of the PDH complex resides in the third subunit of the complex, encoded by the Ipd gene. Therefore, different point mutations have been introduced in the Ipd gene and their effect has been characterized through enzymatic assays and fermentation studies. Three constructs have been created. The first construct $\mathrm{PDH}_{\text {low inhib }}$ carries one AA mutation lowering the NADH inhibition, the second construct PDH $\mathrm{NADP}_{\text {C }}$ carries seven AA mutations changing the cofactor dependency of the PDH complex from NAD ${ }^{+}$to $\mathrm{NADP}^{+}$and the last construct $\mathrm{PDH}_{\mathrm{NADP}+\text { low inhib }}$ carries eight $\mathrm{AA}$ mutations combining the lower NADH inhibition and a change of cofactor. All constructs have been cloned in pBbA2k backbone and expressed in $E$. coli $\Delta / p d$ strain. The enzymatic assays confirmed results obtained in previous work regarding the first two constructs. However, the combination of both reduced sensitivity to NADH and cofactor change has not been attempted before.

The poster shows the enzyme activities of the modified PDH complexes and their effects on E. coli metabolism under both aerobic and anaerobic conditions. Surprisingly, both $\mathrm{PDH}_{\text {NADP }}$ and $\mathrm{PDH}_{\mathrm{NADP}+\text { low inhib }}$ can only use NADP ${ }^{+}$as cofactor and cannot use NAD ${ }^{+}$anymore. Furthermore, not only $\mathrm{PDH}_{\text {NADP }+ \text { low inhib }}$ but also $\mathrm{PDH}_{\text {NADP }}$ was shown to be insensitive to product inhibition by NADH and NADPH. 


\title{
GENETIC TOOL DEVELOPMENT AND SPORULATION MECHANISMS IN THERMOPHILIC CLOSTRIDIA
}

\author{
JOYSHREE GANGULY ${ }^{1}$ (JOYSHREE.GANGULY@CORBION.COM), \\ JOHN VAN DER OOST ${ }^{2}$, "RICHARD VAN KRANENBURG ${ }^{1,2}$ \\ ${ }^{1}$ Corbion - Arkelsedijk 46, 4206 AC Gorinchem, The Netherlands, \\ ${ }^{2}$ Wageningen University, Stippeneng 4, 6708 Wageningen, The Netherlands
}

\section{Introduction}

Sustainable production of bio-based products from biomass is gaining attention. Economic production of green chemicals as building blocks for bioplastics has become a key target. The use of thermophilic clostridia, that can consume and ferment lignocellulose-derived carbohydrates, is an emerging niche in this field. The limited availability of genetic tools for manipulation of clostridial species is a major barrier for exploiting them. Hence, we aim to develop genetic engineering tools for thermophilic clostridia into industrial platform organisms for production of green chemicals.

\section{Objectives}

The goals of the project are: (1) to develop genetic tools for Clostridium thermosuccinogenes, and (2) to study the sporulation mechanism in the same organism.

\section{Materials \& Methods}

The genetic accessibility of $C$. thermosuccinogenes is being studied by electroporation. For the sporulation, a physiological characterisation of the sporulation process in thermophilic clostridia using flow cytometry, FACS, microscopy and Schaeffer-Fulton spore staining has been performed. In addition, the factors $(\mathrm{pH}$, temperature) that potentially trigger sporulation were evaluated.

\section{Results}

C. thermosuccinogenes could reproducibly be transformed by electroporation.

Using flow cytometry, FACS and microscopy, distinct populations of spores, endospores and vegetative cells were enumerated. Factors like temperature, $\mathrm{O}_{2}$ sensitivity play a vital role to trigger sporulation.

\section{Conclusions}

The key aim for this project is to develop $C$. thermosuccinogenes as a platform organism, to boost the production of green chemicals, which can be used in bioplastics industries. The first barrier of genetic accessibility has been taken. Further work is required to establish genome engineering and to eliminate sporulation without affecting central metabolism. 


\title{
METABOLIC ENGINEERING OF THERMOPHILIC CLOSTRIDIA FOR PRODUCTION OF DICARBOXYLIC ACIDS
}

\author{
J.G. KOENDJBIHARIE ${ }^{1,2}$, R. VAN KRANENBURG ${ }^{1}$ \\ ${ }^{1}$ Corbion - Arkelsedijk 46, 4206 AC Gorinchem, The Netherlands \\ ${ }^{2}$ Wageningen University, Stippeneng 4, 6708 Wageningen, The Netherlands
}

\begin{abstract}
Most existing large scale fermentation processes use well studied organisms, such as S. cerevisiae, E. coli, C. glutamicum and A. niger. All of these often-used organisms are socalled mesophilic organisms, which grow at moderate temperatures (30 - 40 degrees). However, in industrial processes it can have many advantages to use thermophilic organisms, which can grow at much higher temperatures. The main advantages are that a process with a thermophile (1) requires less cooling, (2) has a lower risk of contaminations, and ( 3 ) has the possibility to use the biomass-degrading enzymes simultaneously with the fermentation step, simplifying the whole process.
\end{abstract}

Dicarboxylic acids, such as succinic acid have a lot of potential as chemical building blocks for the production of polymers, resins, and solvents. In order to compete with similar, fossil fuelderived products, the production costs need to be very low, which is a big technical challenge. As the potential applications of succinic acid are all very high volume products, solving this challenge might contribute significantly in decreasing our dependence on fossil fuels. And one way to help solve it could be to explore the use of thermophiles.

Project

The aim of the project is the metabolic engineering of thermophilic Clostridia for the production of dicarboxylic acid. More specifically, the project focusses on Clostridium thermosuccinogenes for the production of succinic acid. C. thermosuccinogenes is the only known thermophile that naturally produces large amounts of succinic acid (along with several other products). It is therefore interesting, not just to use $C$. thermosuccinogenes for the production of succinic acid, but also to study its metabolism, as the gained knowledge (or even specific genes) can then be used when engineering other (perhaps better studied/suited) organisms for the production of succinic acid, such as Clostridium thermocellum. 
CHARACTERIZING A THERMOSTABLE CAS9 AND EXPLORING ITS POTENTIAL AS A PROKARYOTIC GENOME ENGINEERING TOOL

I. MOUGIAKOS ${ }^{1}$ (IOANNIS.MOUGIAKOS@WUR.NL),

P. MOHANRAJU ${ }^{1}$, E.F. BOSMA ${ }^{1}$, M. FINGER BOU ${ }^{1}$, V. VROUWE ${ }^{1}$, M. NADUTHODI ${ }^{1}$, A. GUSSAK ${ }^{1}$, R.B.L. BRINKMAN ${ }^{2}$, R. VAN KRANENBURG ${ }^{3}$, J. VAN DER OOST ${ }^{1}$

1 Wageningen University, Microbiology department, The Netherlands 2 Corbion, The Netherlands

3 Wageningen University, Microbiology department, The Netherlands \& Corbion,

The Netherlands

CRISPR-Cas9 based genome engineering tools have revolutionized fundamental research and biotechnological exploitation of both eukaryotes and prokaryotes. However, the mesophilic nature of the already established Cas9 systems restricts their applicability, excluding thermophiles and cellular environments in which mesophilic enzymes are easily inactivated. Here, we identify and characterize Cas9 from the CRISPR-Cas type-IIC system of the thermophilic bacterium Geobacillus thermodenitrificans $\mathrm{T} 12$ as an RNA-guided DNA endonuclease. We provide fundamental insights into a thermophilic CRISPR-Cas family member and describe our work towards the development of a genome editing and silencing tool readily applicable to thermophiles. 


\title{
IMPROVING HETEROLOGOUS PROTEIN PRODUCTION BY TWEAKING TRANSCRIPT ELEMENTS
}

\author{
T. NIEUWKOOP (THIJS.NIEUWKOOP@WUR.NL), \\ J. VAN DER OOST \\ Wageningen University, The Netherlands
}

\begin{abstract}
Optimization of protein production remains a trial and error approach mainly due to our lack of understanding what the key factors are in efficient translation. There is an intrinsic correlation between the mRNA sequence and secondary structures. Synonymous mutations in the coding DNA sequence, for example, introduced by codon harmonization/optimization, can improve translation efficiency. However, they may also create new inhibitory secondary structures that limit translation initiation or slow down the translation elongation process. For an unbiased look at the effects of different transcript regions, each region should act independently, which means that the translation initiation region cannot be hampered by downstream sequence alterations. Unwanted secondary structures cannot be excluded in many of the previous codon studies as the cause of altered protein expression. In this project, a 5'-UTR region is tested that has the potential for independent translation initiation. This region will be used to verify different codon optimization techniques to pinpoint what the limitations are during translation. Finally, the $3^{\prime}$-UTR region is examined and stabilized to protect against exonucleases, increasing the mRNA half-life and subsequent protein expression. The protein expression can be further increased by increasing the promoter strength and the gene copy number. All obtained knowledge is incorporated in so called transcript modules which will be interchanged in combination with the protein expressing region to create a reliable and robust expression platform.
\end{abstract}




\title{
A TRANSCRIPTIONAL REGULATOR-BASED SELECTION SYSTEM FOR OBTAINING NOVEL BIOCATALYSTS
}

\author{
T. VAN ROSSUM (TEUNKE.VANROSSUM@WUR.NL),
} A. MURAS, M.J.J. BAUR, S.C.A. CREUTZBURG, J. VAN DER OOST, S.W.M. KENGEN

Laboratory of Microbiology, Wageningen University and Research, Stippeneng 4, 6708 WE Wageningen, The Netherlands

\begin{abstract}
The use of bioreporters in high-throughput screening for small-molecules is generally laborious and/or expensive. The technology can be simplified by coupling the generation of a desired compound to cell survival, causing only positive cells to stay in the pool of generated variants. Here, a dual selection/screening system was developed for the in vivo detection of novel biocatalysts. The sensor part of the system is based on the transcriptional regulator AraC, which controls expression of both a selection reporter (LeuB or $\mathrm{KmR}$; enabling growth) for rapid reduction of the initially large library size and a screening reporter (LuxCDABE; causing bioluminescence) for further quantification of the positive variants. Out of four developed systems, the best system was the medium copy system with $\mathrm{KmR}$ as selection reporter. As a proof of principle, the system was tested for the selection of cells expressing an L-arabinose isomerase derived from mesophilic Escherichia coli or thermophilic Geobacillus thermodenitrificans. A more than a millionfold enrichment of cells with L-arabinose isomerase activity was demonstrated by selection and exclusion of false positives by screening. This dual selection/screening system is an important step towards an improved detection method for small molecules, and thereby for finding novel biocatalysts.
\end{abstract}




\title{
A NON-CANONICAL SEED SEQUENCE REGULATES TARGETING BY THE CRISPR/CMR COMPLEX
}

YIFAN ZHU, DAVID W. TAYLOR, WILLEM M. DE VOS, RAYMOND J.H. STAALS, JOHN VAN DER OOST

Laboratory of Microbiology

\begin{abstract}
CRISPR-Cas systems in bacteria and archaea provide resistance against mobile genetic elements. Type III systems have been demonstrated to perform RNA-guided RNA and DNA interference. Although recent structural studies have revealed the conserved architecture between Type III and Type I Cascade complexes, each of these multi-subunit effectors recognize and cleave distinct targets. The mechanism by which the interference activity of Type III-B Cmr complex from Thermus thermophilus (TtCmr) is regulated remains unknown. Here, we show that the seed-like region of the guide in TtCmr resides at the $3^{\prime}$ end of the guide, not at its $5^{\prime}$ end like Type I systems. Intriguingly, this non-canonical seed-like region shifts towards the $5^{\prime}$ end as the length of the guide RNA increases. The positioning of the seedlike sequence at the $3^{\prime}$ end of the guide RNA is likely important for interaction with the $5^{\prime}$ end of a nascent transcript targeted by TtCmr, and the ability of the complex to utilize different length guides and shift seeds may contribute to avoiding phage escape.
\end{abstract}


Author index 


\section{Author index}

\section{$\boldsymbol{A}$}

AALVINK, STEVEN ..................................... 62

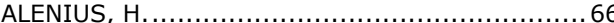

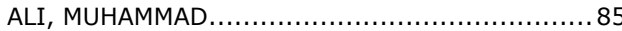

AN, RAN ........................................... 96

ARENDS, JAN B.A. ............................. 48

ATASHGAHI, S. .............................. 61,100

ATASHGAHI, SIAVASH ....................... 84, 95

ATIKANA, AKHIRTA ................................. 97

$\boldsymbol{B}$

BAINES, JOHN F. ............................. 87

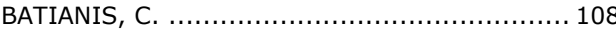

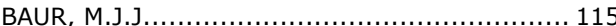

BECKING, LEONTINE E. ..................... 38, 91

BELZER, C. ........................... 64, 89, 101

BELZER, CLARA ...................... 37, 62, 65, 98

BENNINGA, MARC A. ............................. 98

BESSELING-VAN DER VAART, ISOLDE .............96

BLAAK, ELLEN E.................................. 93

BOEKHORST, $] . \ldots \ldots \ldots \ldots \ldots \ldots \ldots \ldots \ldots \ldots \ldots \ldots \ldots . \ldots \ldots$

BOSMA, E.F.................................. 113

BOSMA, ELLEKE F. .............................. 55

BRINKMAN, R.B.L. .............................. 113

BROUNS, STAN ]. J. ...................................... 54

BROUWER, A.................................. 100

$\bar{C}$

CALLAGHAN, M.................................... 86 CAMPOS-QUEVEDO, NOHEMI $\ldots \ldots \ldots \ldots \ldots \ldots \ldots \ldots \ldots$

CANFORA, EMANUEL E............................ 93

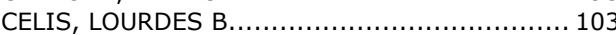

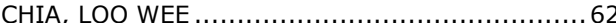

CLAUSSEN, J.C. ................................. 63

CLAUSSEN, JENS CHRISTIAN $\ldots \ldots \ldots \ldots \ldots \ldots \ldots \ldots \ldots$

CREUTZBURG, S.C.A. ............................ 115

CUC, N.T.K....................................... 88

D

DAFFLON, B. ...................................... 41

DAMALAS, S..................................... 108

DAMALAS, STAMATIOS .......................... 109

DAT, T.T.H. .......................................... 88

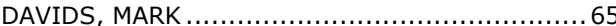

DE BOER, T.E. ................................. 100

DE VOS, W.M. ................................ 64, 89

DE VOS, WILLEM M.........62, 65, 68, 77, 78, 116
DE VRIES, H.J. ................................. 82

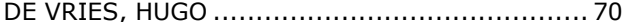

DIENDER, MARTIJN ...........................46, 75

DIEZ DELGADO, IRATXE .......................... 69

DOLFING, JAN $\ldots \ldots \ldots \ldots \ldots \ldots \ldots \ldots \ldots \ldots \ldots \ldots \ldots, 45$

DOS SANTOS, FILIPE BRANCO .................... 50

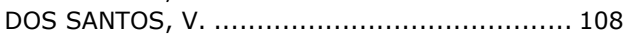

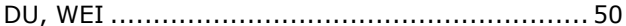

$\bar{E}$

EDWARDS, JOAN E. ........................... 106

EGGINK, G..................................... 110

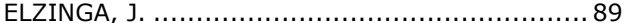

ETTEMA, THIJS .............................. 53

$\boldsymbol{F}$

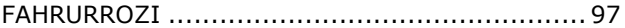

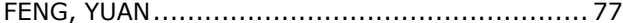

FINGER BOU, M................................ 113

FOLCH, P.L. ........................................ 110

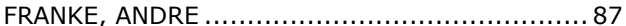

FYHRQUIST, N........................................ 66

$\overline{\boldsymbol{G}}$

GANGULY, JOYSHREE $\ldots \ldots \ldots \ldots \ldots \ldots \ldots \ldots \ldots \ldots . \ldots 111$

GAVRIILIDOU. A ................................. 90

GEERLINGS, S.................................. 64

GEERTS, R........................................... 76

GEORG, STEFFEN................................. 48

GERRITSE, J. ..................................... 61

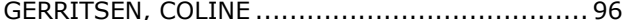

GROOT JEBBINK, L.J.M.......................... 101

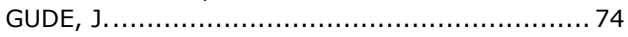

GUEDES DA SILVA, LEONOR ...................... 104

GUSSAK, A. ..................................... 113

GUTLEBEN, J.................................... 90

GUTLEBEN, JOHANNA............................ 42

$\boldsymbol{H}$

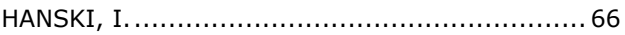

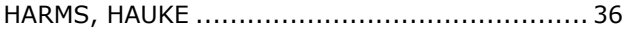

HASSAN, Z.H.

HE, YUJIE ...................................... 51

HEIDENREICH, MATTHIAS......................... 72

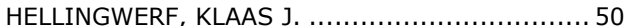

HERMES, GERBEN D.A........................... 93

HORNUNG, B........................................ 61

HORNUNG, BASTIAN ........................... 106 
HORNUNG, BASTIAN V.H.K. ....................... 62

HUBBARD, S. . .................................. 41

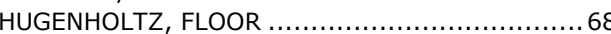

HÜTT, MARC-THORSTEN ......................... 87

$\bar{I}$

IKEDA-OHTSUBO, W. ................. 47 INDRANINGRAT, ANAK AGUNG GEDE .......... 38, 91 INGHAM, C. ...................................... 90

$\bar{J}$

JANSSENS, T.K.S. .............................. 100 JANSSON, J.K..................................... 4

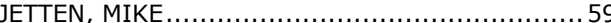

JONGBLOETS, JOERI A. ........................... 50

K

KAPPLER, ANDREAS

KARISOLA

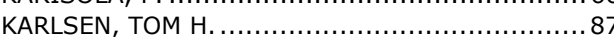

KENGEN, S.W.M. ............................... 115

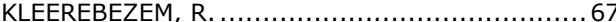

KLEEREBEZEM, ROBBERT....................... 104

KLEIBUSH, E. ................................... 82

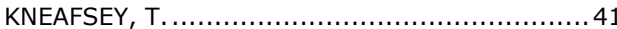

KNOL, J. .................................... 89, 101

KNOL, JAN ................................... 62, 65

KOEHORST, JASPER $\ldots \ldots \ldots \ldots \ldots \ldots \ldots \ldots \ldots \ldots \ldots . \ldots 1$

KOENDJBIHARIE, J.G........................... 112

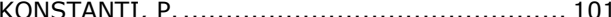

KOSTOPOULOS, I................................ 89

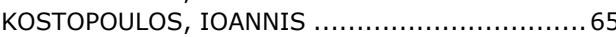

KUSAKA, K ...................................... 47

$\bar{L}$

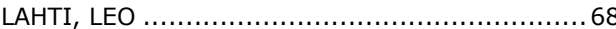

LANGENHOFF, ALETTE ............................. 51

LANGENHOFF, ALETTE A.M.................. 84

LEBBINK, JOYCE H.G............................. 58

LEHTIMÄKI, J. ................................... 66

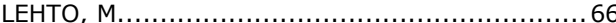

LIAN, JIE $\ldots \ldots \ldots \ldots \ldots \ldots \ldots \ldots \ldots \ldots \ldots \ldots \ldots \ldots \ldots . \ldots 4$

LIEB, WOLFGANG $\ldots \ldots \ldots \ldots \ldots \ldots \ldots \ldots \ldots \ldots \ldots \ldots \ldots$

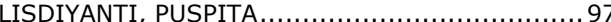

LOPEZ-LOZANO, NGUYEN E...................... 103

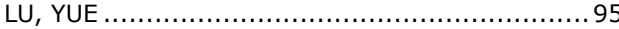

$\bar{M}$

MARTIN, R. ...................................... 101

MARTIN, ROCIO.

39
MASCLEE, AD A.M.................................. 96

MOHANRAJU, P.................................. 113

MOHANRAJU, PRARTHANA ........................... 72

MOLLAEI, MONIR .................................. 73

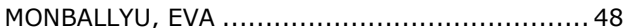

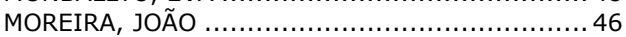

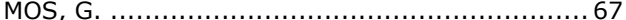

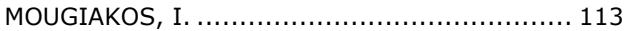

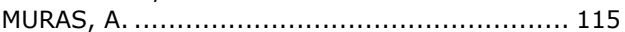

MUYZER, G. ....................................... 79

$\mathbf{N}$

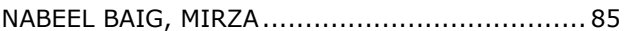

NADUTHODI, M. ................................ 113

NARANJO, HENRY BENAVIDES $. . \ldots \ldots \ldots \ldots \ldots \ldots \ldots . \ldots 1$

NIEUWKOOP, T................................... 114

NIJENHUIS, IVONNE $\ldots \ldots \ldots \ldots \ldots \ldots \ldots \ldots \ldots \ldots \ldots . . . \ldots 40$

NORBRUIS, O.F. .................................. 101

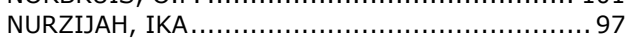

\section{O}

OOSTERKAMP, M.J.

74

OTTMAN, N.

66,89

OTTMAN, NOORA.

. .65

$\boldsymbol{P}$

PALAKAWONG NA AYUDTHAYA, SUSAKUL ......... 78 PENG, PENG .................................... 95 PHILIPS, JO ....................................... 48 PLUGGE, C.M. .................................76, 82 PLUGGE, CAROLINE M............... 73, 78, 79, 106 PRONK, JACK T.................................. 49

$\bar{R}$

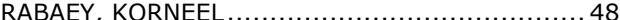

RAMIRO-GARCIA, JAVIER .....................84, 95

RATNAKOMALA, SHANTI .......................... 97

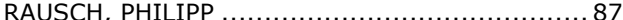

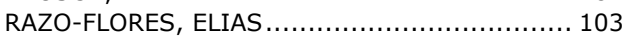

RENES, I.B.................................... 101

RIJKERS, GERT T. ........ 96

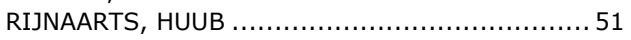

RIJNAARTS, HUUB H.M.......................... 84

ROMBOUTS, J.

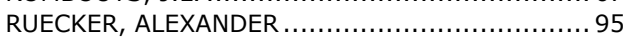

RUOKOLAINEN, L.................................... 66

\section{$\bar{S}$}

S'ANCHEZ-ANDREA, IRENE $\ldots \ldots \ldots \ldots \ldots \ldots \ldots \ldots 77$

SACCENTI, EDOARDO ............................. 84 
SALES E SANTOS LOUREIRO,

CATARINA CAROLINA ..........................4 42

SÁNCHEZ-ANDREA, I..................................80, 105

SÁNCHEZ-ANDREA，IRENE $\ldots \ldots \ldots \ldots \ldots \ldots \ldots \ldots \ldots \ldots$

SANDERS, J.P.M. .................................... 110

SARI, MIRANTI NURINDAH .......................... 97

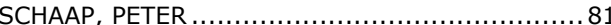

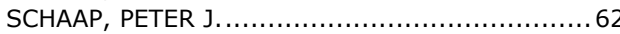

SCHINK, BERNHARD …........................... 44

SCHOLS, H.A …….................................. 92

SCHOLS, H.A. (HENK) ................................ 99

SCHOTERMAN, MAGRGRIET H.C. .................... 98

SCHUPP, PETER J .................................... 38

SEGUI PEREZ, CELIA ….............................. 96

SHAHID, AHMAD ALI $\ldots \ldots \ldots \ldots \ldots \ldots \ldots \ldots \ldots \ldots \ldots . \ldots \ldots$

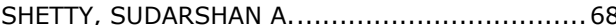

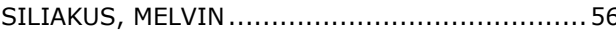

SIPKEMA, D. ................................ 88, 90

SIPKEMA, DETMER ............ 38, 42, 91, 94, 95, 97

SKIECEVICIENE, JURGITA ........................... 87

SLEUTELS, TOM H.J.A................................ 73

SMIDT, H........................ 61, 88, 90, 92, 100

SMIDT, HAUKE .. 38, 42, 68, 69, 70, 84, 91, 94, 95,

96, 97, 99, 106

SOROKIN, D.1\%1,

SOUSA, DIANA Z. ............................. 46, 75, 81

STAALS, RAYMOND J.H............................. 116

STAMS, A.J.M. .......................61, 80, 100, 105 STAMS, ALFONS J.M....... 46, 73, 75, 77, 78, 79, 81 STEINERT, G..................................... 88

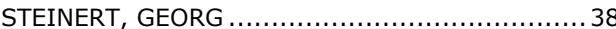

STREPIS, NIKOLAOS ….................................... 81

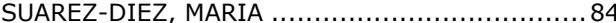

SUOMALAINEN, A. ...................................... 66

SUTTON, NORA …....................................... 51

SUTTON, NORA B...................................... 84

$\boldsymbol{T}$

TAŞ, N......................................................... 41

TAYLOR， DAVID W. ..................................... 116

TAYLOR, MICHAEL W.

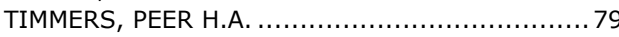

TRINGE, S.G. ........................................ 41

TROOST, FRED J.................................. 96

\section{$\boldsymbol{U}$}

ULRICH, C...

UMANETS, ALEXANDER

$\ldots . .41$

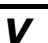

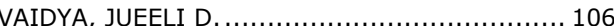

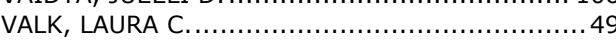

VAN DE WEIJER, ANTONIUS H.P. ................. 78

VAN DEN BRINK, P.............................. 82

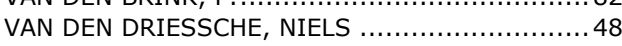

VAN DER ARK, K................................... 64

VAN DER GRAAF, C.M........................... 105

VAN DER MEER, JAN ROELOF .................... 33

VAN DER OOST, J......................113, 114, 115

VAN DER OOST, JOHN ................... 72, 111, 116

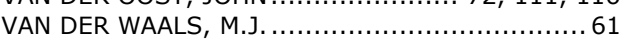

VAN GELDER, A.H.............................. 100

VAN GELDER, ANTONIE H. ..................... 78

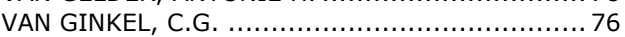

VAN HALEM, D. .................................. 74

VAN HECK, R................................... 108

VAN KRANENBURG, R.....................112, 113

VAN KRANENBURG, RICHARD $\ldots \ldots \ldots \ldots \ldots \ldots \ldots \ldots \ldots \ldots \ldots$

VAN LINGEN, R.A.............................. 101

VAN LOOSDRECHT, M.C.M. ......................... 67

VAN LOOSDRECHT, MARK ........................... 34

VAN LOOSDRECHT, MARK C.M...............49, 104

VAN ROSSUM, T. ................................ 115

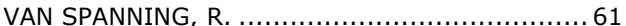

VAN VLIET, D.M.................................. 80

VAN ZOEREN-GROBBEN, D. ..................... 101

VAUGHAN, ELAINE E. ........................... 98

VAVOURAKIS, C. ................................. 79

VENEMA, KOEN .................................. 93

VERHAGEN, KOEN................................... 104

VERSLUIS, D..................................... 90

VILLANUEVA, L.................................... 80

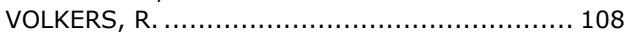

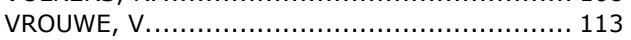

\section{$\mathbf{M}$}

WAHL, ALJOSCHA S. ............................. 104

WANG, JUN ........................................ 87

WEGH, CARRIE A.M. ............................... 98

WEISSBRODT, D.G. .................................. 67

WELLS, JERRY .................................. 70

WELLS-BENNIK, M.H.J............................ 86

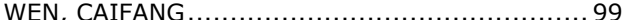

WEUSTHUIS, R.A.............................. 110

WEUSTHUIS, RUUD A............................... 57

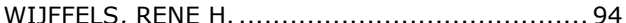

WILMS, ELLEN .................................... 96

WIRANATA WIJAYA, ANDY ........................ 104

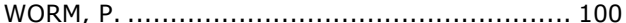

WU, WEN Y...................................... 72

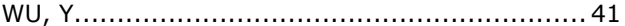

$\bar{Z}$

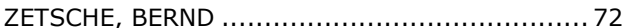

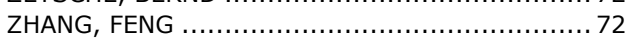

ZHENG, Y. ............................................ 100

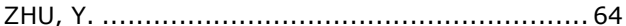

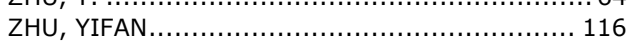

ZOETENDAL, E.G. .............................. 92

ZOETENDAL, ERWIN G. .......................93, 96

ZWITTINK, R.D................................ 101 